
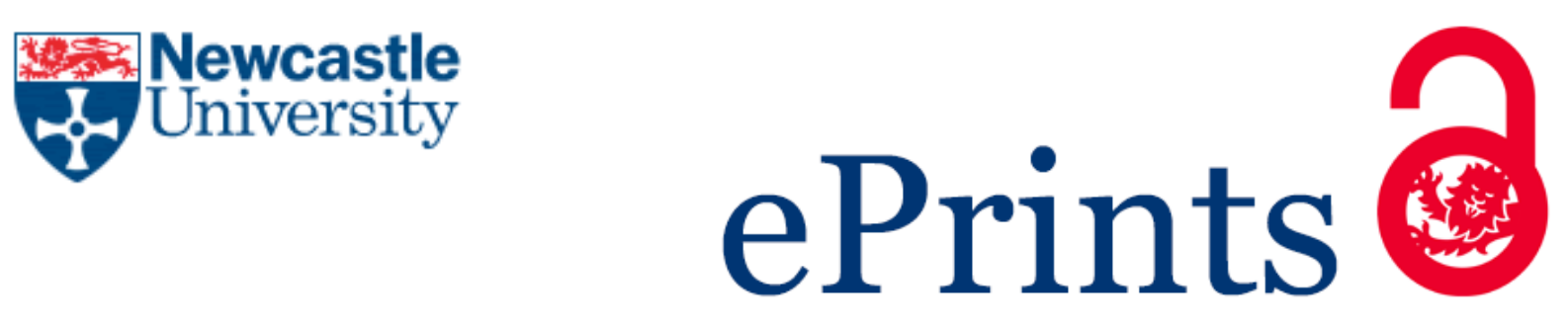

Zaytsev A, Dodd B, Magnani M, Ghiron C, Golding BT, Griffin RJ, Liu J, Lu X, Micco I, Newell DR, Padova A, Robertson G, Lunec J, Hardcastle IR. Searching for Dual Inhibitors of the MDM2-p53 and MDMX-p53 Protein-Protein Interaction by a Scaffold-Hopping Approach. Chemical Biology and Drug Discovery 2015, 86(2), 180-189.

\title{
Copyright:
}

This is the peer reviewed version of the following article: Zaytsev A, Dodd B, Magnani M, Ghiron C, Golding BT, Griffin RJ, Liu J, Lu X, Micco I, Newell DR, Padova A, Robertson G, Lunec J, Hardcastle IR. Searching for Dual Inhibitors of the MDM2-p53 and MDMX-p53 Protein-Protein Interaction by a ScaffoldHopping Approach. Chemical Biology and Drug Discovery 2015, 86(2), 180-189. which has been published in final form at http://dx.doi.org/10.1111/cbdd.12474. This article may be used for non-commercial purposes in accordance with Wiley Terms and Conditions for Self-Archiving.

DOI link to article:

http://dx.doi.org/10.1111/cbdd.12474

Date deposited:

$09 / 03 / 2016$

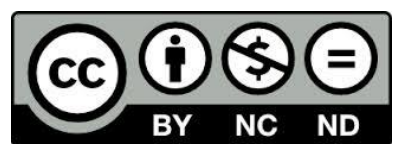

This work is licensed under a

Creative Commons Attribution-NonCommercial-NoDerivatives 4.0 International licence 


\section{Searching for dual inhibitors of the MDM2-p53 and MDMX- p53 protein-protein interaction by a scaffold-hopping approach}

Andrey Zaytsev, ${ }^{1}$ Barry Dodd, ${ }^{1}$ Matteo Magnani, ${ }^{3}$ Chiara Ghiron, ${ }^{3}$ Bernard T. Golding, ${ }^{1}$ Roger J. Griffin, ${ }^{1}$ Junfeng Liu, ${ }^{2}$ Xiaohong Lu, ${ }^{2}$ Iolanda Micco, ${ }^{3}$ David R. Newell, ${ }^{2}$ Alessandro Padova, ${ }^{3}$ Graeme Robertson, ${ }^{3}$ John Lunec, ${ }^{2}$ lan R. Hardcastle ${ }^{1^{*}}$

${ }^{1}$ Newcastle Cancer Centre, Northern Institute for Cancer Research and School of Chemistry, Bedson Building, Newcastle University, Newcastle, NE1 7RU, UK

${ }^{2}$ Newcastle Cancer Centre, Northern Institute for Cancer Research, Paul O'Gorman Building, Medical School, Framlington Place, Newcastle University, Newcastle, NE2 4HH, UK

${ }^{3}$ Siena Biotech S.p.A., Strada del Petriccio e Belriguardo, 35, Siena, 53100, Italy 


\section{ABSTRACT}

Two libraries of substituted benzimidazoles were designed using a 'scaffold-hopping' approach based on reported MDM2-p53 inhibitors. Substituents were chosen following library enumeration and docking into an MDM2 X-ray structure. Benzimidazole libraries were prepared using an efficient solution-phase approach, and screened for inhibition of the MDM2-p53 and MDMX-p53 protein-protein interactions. Key examples showed inhibitory activity against both targets.

\section{INTRODUCTION}

The design of small molecule inhibitors of protein-protein interactions has received considerable interest in recent years.(1-4) In particular, the interaction of p53 with the regulatory proteins MDM2 and MDMX have provided a focus for efforts because of their importance in a number of cancers, and the prospect that inhibition of these interactions may provide new therapies. Overexpression of the regulatory proteins MDM2 and MDMX has been demonstrated to silence the tumor suppressor function of p53. MDM2 amplification occurs in around $11 \%$ of all tumors, and is most common in hepatocellular carcinoma (44\%), osteosarcomas (20\%), and soft tissue sarcomas (31\%). Similarly, MDMX (MDM4) occurs in approximately 10$25 \%$ of tumors, e.g. brain (11\%), breast (5-40\%), and sarcomas (17\%).(5-8)

Expression of MDM2 is p53-dependent, and forms a regulatory feedback loop with p53 by binding to the p53 transactivation domain. Acting as an E3-ligase, MDM2 promotes proteasomal degradation of p53.(912) MDMX expression is not $p 53-$ dependent and MDMX levels remain constant. The protein inhibits $p 53$ transcriptional activity, but does not act as an E3 ligase independently of MDM2.(13) Thus, MDM2 and MDMX both regulate $p 53$, but their functions are different and non-redundant.(14)

The X-ray crystal structure of MDM2 bound to a p53-derived peptide reveals the $\alpha$-helical peptide bound into a deep groove on the surface of the protein, and key interactions are formed with three hydrophobic residues of p53 (Figure 1).(15) Inhibitors of MDM2 with diverse chemotypes have been reported. $(16,17)$ X-ray crystal structures of a range of potent MDM2-p53 inhibitors have been published, such as Nutlin-3 (1), spirooxindoles, e.g. MI-63 (2) and pyrrolidones (3).(18-21) Inhibitors show cellular activity and in vivo antitumor activity. $(18,22)$ Recently, potent MDM2-p53 inhibitors have entered clinical trials, including RG7112,(23, 24) RG7388(25) and MI-773(26) (Chart 1).

$<$ figure 1, chart $1>$

The $\mathrm{p} 53$ transactivation loop binds in a cleft on the surface of MDMX similar to that identified for MDM2 but with a closed conformation for Tyr99.(27, 28). Steric hindrance of Met53 in the Leu23 pocket for MDMX may be responsible for the lack of potency against MDMX for MDM2-p53 inhibitors such as the Nutlins and spirooxindoles.(29) A high degree of flexibility has been measured in computational studies of MDMX, and significant conformational differences, owing to the induced fit of ligands are observed in crystal 
structures.(30-32) Reports of small-molecule MDMX-p53 inhibitors have not been as numerous as for MDM2.(21) The selective MDMX inhibitor 5-oxo-pyrazolylidene SJ-172550 (4) was identified in an MDMX high-throughput assay.(33) The 3-imidazolylindole 5 is a mixed MDM2- and MDMX-p53 inhibitor with modest potency against MDMX.(29) Recently, indolylhydantoins, e.g. (6) have been reported as MDM2-p53 and MDMX-p53 inhibitors.(34)

$<$ structures 4,5,6>

In this paper, we describe the application of a scaffold-hopping approach to design new inhibitor chemotypes for $\operatorname{MDM} 2$ and $\operatorname{MDMX}(35,36)$ based on heterocycle replacement, and derived from two MDM2-p53 inhibitors with significantly different core structures. In particular, starting from cis-imidazoline (1),(18) and a class of oxindoles $(7),(37,38)$ benzimidazole derivatives were designed and evaluated through docking studies. The benzimidazole heterocycle was chosen as a 'privileged structure' in medicinal chemistry that is readily synthetically accessible.(39) The synthesis of small libraries yielded mixed MDM2-p53 and MDMX-p53 inhibitors with promising potency.

<structure 7>

\section{MATERIALS AND METHODS}

MDM2-p53 ELISA assay. Compounds were assayed for MDM2-p53 inhibitory activity using ELISA assays in a 96-well format using the published method.(40)

MDMX-p53 ELISA assay. MDMX-p53 inhibitory activity was determined using an analogous method incorporating a pCMV-XL5-MDMX cDNA construct (OriGene Technologies) for the in vitro coupled T7 transcription and rabbit reticulocyte lysate translation of $M D M X$, and a rabbit anti-MDMX antigen affinitypurified polyclonal antibody (Bethyl Laboratories Inc, via UK supplier Cambridge Bioscience, UK, Cat No. A300-287 A).(41)

Docking experiments. Docking calculations were performed with the GOLD software.(42) Ligands were docked within the p53 binding site of MDM2, using the crystal structure of human MDM2 in complex with the small molecule inhibitor Nutlin-2 (PDB code: 1RV1).(18) A single protein chain was selected from the unit cell. The binding site was defined by hydrophobic fitting points calculated on the target for a $8 \AA$ radius around the co-crystallized ligand. GoldScore was used as a fitness function, while default genetic algorithm parameter settings were applied. 30 poses were generated for each ligand, early termination being allowed when the top 10 solutions were within 1.5 ̊ R.M.S.D.

\section{Chemical Synthesis.}

\section{General Procedure A - Synthesis of compounds (14).}

A mixture of the appropriate aldehyde (1.2 eq.), sodium dithionite ( $86 \%$ purity; $1.98 \mathrm{~g}, 9.78 \mathrm{mmol}, 3$ eq.) and $13(1.16 \mathrm{~g}, 3.26 \mathrm{mmol}, 1.0$ eq.) in methanol $(12 \mathrm{~mL})$ and water $(3 \mathrm{~mL})$ was heated in a microwave reactor for 10 minutes at $100{ }^{\circ} \mathrm{C}$. The sample was diluted with ethyl acetate $(20 \mathrm{~mL})$ and washed with water $(20 \mathrm{~mL})$ 
and brine $(20 \mathrm{~mL})$. The organic layer was dried $\left(\mathrm{MgSO}_{4}\right)$, and evaporated. Chromatography (silica; ethyl acetate, petrol) gave compound 14.

General Procedure B. Compound 14 was dissolved in DCM $(2 \mathrm{~mL})$ and TFA $(3 \mathrm{~mL})$ and the mixture stirred at rt for 1 hour, then evaporated. The residue was diluted with DCM $(20 \mathrm{~mL})$ and neutralised with sodium carbonate (sat.; $20 \mathrm{~mL}$ ). The organic layer was dried $\left(\mathrm{MgSO}_{4}\right)$ to give 15 which was used without further purification.

General procedure C. Either an aliquot of a solution of 15 in dry DCM (1 mL, $0.049 \mathrm{M})$, or weighed 15 in dry $\mathrm{DCM}(1 \mathrm{~mL})$ was treated with the named isocyanate (1.5 eq.). The mixture was quenched with $\mathrm{NH}_{2}$-silica and filtered through a plug of $\mathrm{NH}_{2}$-silica. Evaporation gave $\mathbf{8}$ without further purification.

(S)-Ethyl 3-(3-(5-chloro-2-(3-methoxybenzyl)-1H-benzo[d]imidazol-1-yl)piperidine-1carboxamido)benzoate $8\{3,7\}$.

General procedure C: $15\{3\}(1 \mathrm{~mL}, 49.3 \mu \mathrm{mol})$ and ethyl 3-isothiocyanatobenzoate $(12 \mu \mathrm{l}, 76 \mu \mathrm{mol}) . \mathbf{8}\{3,7\}$ (26 mg, 96\%) as a white solid; HPLC purity (as area \%) > 98; ${ }^{1} \mathrm{H} \mathrm{NMR}\left(300 \mathrm{MHz}, \mathrm{CDCl}_{3}\right) 1.38(1 \mathrm{H}, \mathrm{t}, J 7.2$, $\left.\mathrm{CH}_{2} \mathrm{CH}_{3}\right), 1.54(1 \mathrm{H}, \mathrm{qt}, J 3.6$ and 13.1), $1.72(1 \mathrm{H}$, app.dd), $1.87(1 \mathrm{H}, \mathrm{app} . \mathrm{d}), 2.20(1 \mathrm{H}, \mathrm{qd}, J 4.0$ and 12.7$), 2.80$ $\left(1 \mathrm{H}, \mathrm{td}, J 2.2\right.$ and 13.1), 3.38(1H, app.t), $3.63\left(3 \mathrm{H}, \mathrm{s}, \mathrm{OCH}_{3}\right), 3.77$ (1H, app. dd), $4.21(1 \mathrm{H}, \mathrm{app} . \mathrm{d}), 4.30$ (2H, s, $\left.\mathrm{CH}_{2}\right), 4.36(2 \mathrm{H}, \mathrm{q}, J$ J 7.2), $6.54(1 \mathrm{H}, \mathrm{br} . \mathrm{d}), 6.65-6.69(1 \mathrm{H}, \mathrm{m}, \mathrm{Ar}), 6.77(2 \mathrm{H}, \mathrm{d}, J$ 8.3, Ar), 7.17 (1H, app.t, Ar), 7.20 $(1 \mathrm{H}, \mathrm{m}, \mathrm{Ar}), 7.32-7.39(2 \mathrm{H}, \mathrm{m}, \mathrm{Ar}), 7.67-7.81(4 \mathrm{H}, \mathrm{m}, \mathrm{Ar}) ;{ }^{13} \mathrm{C} \mathrm{NMR}\left(100 \mathrm{MHz}, \mathrm{CDCl}_{3}\right)$ 14.3, 24.8, 28.6, 35.0, 44.1 , 47.2, 53.5, 55.2, 61.1, 112.2, 112.5, 114.3, 119.8, 120.5, 121.0, 122.7, 124.4, 124.7, 127.7, 128.9, 129.9, $131.0,132.1,137.5,138.9,144.0,154.2,154.7,160.0,166.3$.

\section{(S)-Ethyl 3-(3-(5-chloro-2-(4-methoxybenzyl)-1H-benzo[d]imidazol-1-yl)piperidine-1-} carboxamido)benzoate $8\{4,7\}$.

General procedure C: $15\{4\}$ (1 mL, $49 \mu \mathrm{mol})$ and 3-isocyanatobenzoate $(12 \mu \mathrm{l}, 76 \mu \mathrm{mol}) .8\{4,7\}(23 \mathrm{mg}, 85 \%)$, white solid; HPLC purity (as area \%) > 98; ${ }^{1} \mathrm{H}$ NMR $\left(400 \mathrm{MHz}, \mathrm{CDCl}_{3}\right) 1.33\left(3 \mathrm{H}, \mathrm{t}, J 4.1, \mathrm{CH}_{2} \mathrm{CH}_{3}\right), 1.48(1 \mathrm{H}$, qt, $J$ 4.1 and 13.3), 1.62 (1H, app.d), $1.82(1 \mathrm{H}$, app.d), 2.17 (1H, qd, J 4.1 and 12.8), $2.78(1 \mathrm{H}, \mathrm{td}, J 2.3$ and 13.3), $3.36(1 \mathrm{H}, \mathrm{app} . \mathrm{t}), 3.55\left(3 \mathrm{H}, \mathrm{s}, \mathrm{OCH}_{3}\right), 3.82(1 \mathrm{H}, \mathrm{dd}, J 4.1$ and 12.8), $4.15(1 \mathrm{H}, \mathrm{d}, J 16.0), 4.17$ (1H, app.d), 4.23 $\left(1 \mathrm{H}, \mathrm{d}, J\right.$ 16.0), 4.25-4.33 (1H, m), $4.30\left(2 \mathrm{H}, \mathrm{q}, J\right.$ J 7.3, $\left.\mathrm{CH}_{2} \mathrm{CH}_{3}\right), 6.70-6.73(3 \mathrm{H}, \mathrm{app} . \mathrm{d}), 7.04(2 \mathrm{H}, \mathrm{d}, J$ 8.7, $\mathrm{Ar}), 7.15$ $(1 \mathrm{H}, \mathrm{dd}, J 1.8$ and 8.7), 7.28-7.33 (2H, m, Ar), 7.59-7.62 (1H, m, Ar), 7.63-7.70 (2H, m, Ar), 7.81 (1H, app.d, Ar); ${ }^{13} \mathrm{C} \mathrm{NMR}\left(100 \mathrm{MHz}, \mathrm{CDCl}_{3}\right)$ 14.3, 24.9, 28.6, 34.1, 44.2, 47.2, 53.4, 55.0, 61.0, 112.2, 114.2, 119.7, 121.1, $124.4,124.8,127.6,127.7,128.9,129.4,131.0,132.2,139.0,144.0,154.7,154.9,158.7,166.3 ;$ MS (ESI+) m/z $=547.3[\mathrm{M}+\mathrm{H}]^{+}$.

\section{(S)-N-Benzyl-3-(2-benzyl-5-chloro-1H-benzo[d]imidazol-1-yl)piperidine-1-carbothioamide 16.}

General procedure C: $15\{1\}(16 \mathrm{mg}, 49.2 \mu \mathrm{mol})$ and benzyl isothiocyanate $(7.2 \mu \mathrm{l}, 54.1 \mu \mathrm{mol}) .16$ (15 mg, $64 \%$ ) as a white solid; HPLC purity (as area \%) > 93; ${ }^{1} \mathrm{H} \mathrm{NMR}\left(500 \mathrm{MHz}, \mathrm{CDCl}_{3}\right) 1.43(1 \mathrm{H}$, qt, J 4.1 and 13.3), $1.55(1 \mathrm{H}$, app.d), $1.77-1.80(1 \mathrm{H}, \mathrm{m}), 2.25(1 \mathrm{H}, \mathrm{qd}, J 12.8$ and 4.4$), 3.03(1 \mathrm{H}, \mathrm{td}, J 13.4$ and 2.4$), 3.50(1 \mathrm{H}$, 
app.t), 4.35-4.46 (4H, m), 4.67-4.71 (1H,m), $4.74(1 \mathrm{H}, \mathrm{dd}, J 14.6$ and 4.6), $4.97(1 \mathrm{H}, \mathrm{dd}, J 14.6$ and 5.4), 5.65 (1H, app.t, NH), 7.15-7.22 (6H, m, Ar), 7.30-7.36 (6H, m, Ar), $\left.7.73(1 \mathrm{H}, \mathrm{d}, J 2.0, \mathrm{Ar}) ;{ }^{13} \mathrm{C} \mathrm{NMR} \mathrm{(125} \mathrm{MHz,} \mathrm{CDCl}{ }_{3}\right)$ $24.5,28.4,35.0,48.0,50.4,51.3,52.7,112.0,119.9,122.7,127.2,127.7,127.8,127.9,128.4,128.8,128.9$, $132.1,136.2,137.8,144.1,154.5,183.2$. LC-MS (ESI+) $\mathrm{m} / \mathrm{z}=475.3[\mathrm{M}+\mathrm{H}]^{+}$.

\section{(S)- 2-Benzyl-1-(1-(benzylsulfonyl)piperidin-3-yl)-5-chloro-1H-benzo[d]imidazole 17.}

Phenylmethanesulfonyl chloride (12 mg, $60.9 \mu \mathrm{mol}, 1.1 \mathrm{eq}$ ) was added to a solution of 15 (18 mg, $55.4 \mu \mathrm{mol}$, 1eq) and triethylamine $(11.6 \mu \mathrm{l}, 83.1 \mu \mathrm{mol}, 1.5 \mathrm{eq})$ in DCM $(1 \mathrm{~mL})$. The mixture was stirred at RT for $1 \mathrm{~h}$, then quenched with water $(3 \mathrm{~mL})$. The organic layer was separated, dried $\left(\mathrm{MgSO}_{4}\right)$, and evaporated. Chromatography (5\% DCM, methanol) gave 17 (25 mg, 94\%) as a white solid; HPLC purity (as area \%) > 96; ${ }^{1} \mathrm{H}$ $\operatorname{NMR}\left(500 \mathrm{MHz}, \mathrm{CDCl}_{3}\right)$ 1.34-1.41 (2H, m), 1.64-1.68 (1H, m), $1.94(1 \mathrm{H}, \mathrm{qd}, J 12.6$ and 4.2), $2.54(1 \mathrm{H}, \mathrm{td}, J 12.6$ and 2.6), $3.01(1 \mathrm{H}$, app.t), $3.54(1 \mathrm{H}, \mathrm{dd}, J 12.3$ and 4.6$), 3.61-3.64(1 \mathrm{H}, \mathrm{m}), 4.13(1 \mathrm{H}, \mathrm{d}, J 14.1), 4.17(1 \mathrm{H}, \mathrm{d}, J$ 14.1), $4.19(1 \mathrm{H}, \mathrm{d}, J$ 15.9), $4.24(1 \mathrm{H} ; \mathrm{tt}, J 12.0$ and 4.1), $4.40(1 \mathrm{H}, \mathrm{d}, J$ 15.9), $7.11(1 \mathrm{H}, \mathrm{d}, J$ 8.7, Ar), $7.14(1 \mathrm{H}, \mathrm{dd}$, J 8.7 and 1.8, $\mathrm{Ar}), 7.18-7.23(3 \mathrm{H}, \mathrm{m}, \mathrm{Ar}), 7.27-7.30(3 \mathrm{H}, \mathrm{m}, \mathrm{Ar}), 7.32-7.37(4 \mathrm{H}, \mathrm{m}, \mathrm{Ar}), 7.72(1 \mathrm{H}, \mathrm{d}, J$ 1.8, $\mathrm{Ar})$; LC-MS (ESI+) $\mathrm{m} / \mathrm{z}=480.3[\mathrm{M}+\mathrm{H}]^{+}$.

\section{General Procedure E: Benzimidazole Intermediate Synthesis (20)}

A mixture $18(0.3 \mathrm{~g}, 1.41 \mathrm{mmol})$, sodium dithionite $(0.67 \mathrm{~g}, 3.84 \mathrm{mmol})$ and 19 (1.0 eq.) in methanol (16 mL) and water $(4 \mathrm{~mL})$ was heated by microwave for 10 mins at $100{ }^{\circ} \mathrm{C}$, then diluted with ethyl acetate $(20 \mathrm{~mL})$, washed with water $(20 \mathrm{~mL})$ and brine $(20 \mathrm{~mL})$, dried $\left(\mathrm{MgSO}_{4}\right)$ and evaporatated. Recrystallisation from ethyl acetate, petrol gave 20.

\section{General Procedure F: Boc deprotection (21)}

A mixture of $20(0.40 \mathrm{~g}, 0.94 \mathrm{mmol})$, TFA $(0.6 \mathrm{~mL})$ and DCM $(2 \mathrm{~mL})$ was stirred at $\mathrm{rt}$ for $4.5 \mathrm{~h}$, then concentrated and diluted with methanol $(4 \mathrm{~mL}) . \mathrm{K}_{2} \mathrm{CO}_{3}(0.2 \mathrm{~g})$ was added and the suspension was stirred at $\mathrm{rt}$, then filtered, and concentrated. Chromatography (5\% DCM, MeOH) gave 21. Intermediates $\mathbf{2 1}\{2\}, \mathbf{2 1}\{3\}$, $\mathbf{2 1}\{4\}$ were used directly in the subsequent step without purification.

\section{General Procedure G: Benzimidazole Synthesis (9)}

$21(0.01 \mathrm{~g}, 0.028 \mathrm{mmol})$ was added to a solution of the appropriate isocyanate $(1.2 \mathrm{eq}$.) in $\mathrm{DCM}(2 \mathrm{~mL})$ and the mixture was stirred at $\mathrm{rt}$ for $1 \mathrm{~h}$, then concentrated. Chromatography ( $\mathrm{NH}$-silica; $2 \% \mathrm{MeOH}, \mathrm{DCM}$ ) gave 9 as glassy solids.

\section{$N$-(4-Acetylphenyl)-4-(6-chloro-1-(3-chlorobenzyl)-1H-benzo[d]imidazole-2-yl)piperidine-1-carboxamide} $9\{2,2\}$

General Procedure G: 21\{2\} (0.011 g, 0.029mmol), 4-acetylphenylisocyanate (0.005 g, $0.032 \mathrm{mmol}) .9\{2,2\}$ 81\%; HPLC purity (as area \%) > 99; ${ }^{1} \mathrm{H}$ NMR (400 MHz , $\left.\mathrm{CDCl}_{3}\right) 1.88\left(\mathrm{~m}, 2 \mathrm{H}, \mathrm{CH}_{2} \mathrm{CH}\right), 2.09\left(\mathrm{~m}, 2 \mathrm{H}, \mathrm{CH}_{2} \mathrm{NCO}\right)$, 2.49, (s, 3H, $\left.\mathrm{COCH}_{3}\right) 2.99\left(\mathrm{~m}, 2 \mathrm{H}, \mathrm{CH}_{2} \mathrm{NCO}\right), 3.07\left(\mathrm{~m}, 1 \mathrm{H}, \mathrm{CH}_{2} \mathrm{CH}\right), 4.17\left(\mathrm{~m}, 2 \mathrm{H}, \mathrm{CH}_{2} \mathrm{CH}_{2} \mathrm{NCO}\right), 5.31(\mathrm{~s}, 2 \mathrm{H}$, $\left.\mathrm{CH}_{2} \mathrm{Ar}\right), 6.49(\mathrm{~s}, 1 \mathrm{H}, \mathrm{N}-\mathrm{H}), 6.85(\mathrm{~m}, 1 \mathrm{H}, \mathrm{Ar}-\mathrm{H}), 7.06(\mathrm{~s}, 1 \mathrm{H}, \mathrm{Ar}-\mathrm{H}), 7.14(\mathrm{~d}, 2 \mathrm{H}, J 8.37 \mathrm{~Hz}, \mathrm{Ar}-\mathrm{H}) 7.17(\mathrm{~d}, 1 \mathrm{H}, J 1.82$ 
$\mathrm{Hz}, \mathrm{Ar}-\mathrm{H}), 7.39$ (d, 3H, J 7.91 Hz Ar-H), 7.38 (d, 2H, J $8.85 \mathrm{~Hz}, \mathrm{Ar}-\mathrm{H}), 7.81$ (d, 1H, J $8.56 \mathrm{~Hz}, \mathrm{Ar}-\mathrm{H})$; LC-MS (ESI+) $m / z=519[\mathrm{M}+\mathrm{H}]^{+}$.

\section{4-(6-Chloro-1-(3-chlorobenzyl)-1H-benzo[d]imidazol-2-yl)-N-(3,4,5-trimethoxyphenyl)piperidine-1-} carboxamide $9\{2,3\}$

General Procedure G: $21\{2\}$ (0.05 g, $0.14 \mathrm{mmol})$, 3,4,5-trimethoxyphenylisocyanate $(0.035 \mathrm{~g}, 0.16 \mathrm{mmol})$. 9\{2,3\} $73 \%$; HPLC purity (as area \%) > 92; ${ }^{1} \mathrm{H}$ NMR $\left(400 \mathrm{MHz}, \mathrm{CDCl}_{3}\right) 1.82\left(\mathrm{~m}, \mathrm{br}, 2 \mathrm{H}, \mathrm{CH}_{2} \mathrm{CH}_{2}-\mathrm{NH}\right), 2.10(\mathrm{~m}$, $\left.2 \mathrm{H}, \mathrm{CH}_{2} \mathrm{CH}_{2}-\mathrm{NH}\right), 2.99\left(\mathrm{~m}, 3 \mathrm{H}, \mathrm{CH}_{2}-\mathrm{CH}_{2}-\mathrm{NH}, \mathrm{CH}-\mathrm{CH}_{2}\right), 3.73(\mathrm{~s}, 3 \mathrm{H}, \mathrm{Ar}-\mathrm{OMe}), 3.77(\mathrm{~s}, 6 \mathrm{H}, \mathrm{Ar}-(\mathrm{OMe}) 2), 4.12(\mathrm{~m}$, $\left.2 \mathrm{H}, \mathrm{CH}_{2}-\mathrm{N}\right), 5.27\left(\mathrm{~s}, 2 \mathrm{H}, \mathrm{CH}_{2}-\mathrm{N}\right), 6.31(\mathrm{~s}, 1 \mathrm{H}, \mathrm{N}-\mathrm{H}), 6.59(\mathrm{~s}, 2 \mathrm{H}, \mathrm{Ar}-\mathrm{H}), 6.72(\mathrm{~d}, 1 \mathrm{H}, J 7.21, \mathrm{Ar}-\mathrm{H}), 7.01(\mathrm{~s}, 1 \mathrm{H}, \mathrm{Ar}-$ H), $7.13(\mathrm{~m}, 3 \mathrm{H}, \mathrm{Ar}-\mathrm{H}), 7.62$ (d, $1 \mathrm{H}, 8.33 \mathrm{~Hz}, \mathrm{Ar}-\mathrm{H})$; LC-MS (ESI+) $\mathrm{m} / \mathrm{z} 569[\mathrm{M}+\mathrm{H}]^{+}$;

Ethyl-4-(4-(6-chloro-1-(3-chlorobenzyl)-1H-benzo[d]imidazole-2-yl)piperidine-1-carboxamido) benzoate $9\{2,8\}$

General Procedure G: $21\{2\}$ (0.011 g, $0.029 \mathrm{mmol})$, ethyl 4-isocyanobenzoate (0.006 g, $0.032 \mathrm{mmol}) .9\{2,8\}$ 80\%; HPLC purity (as area \%) > 98; ${ }^{1} \mathrm{H}$ NMR $\left(400 \mathrm{MHz}, \mathrm{CDCl}_{3}\right) 1.40\left(\mathrm{t}, 3 \mathrm{H}, 7.11 \mathrm{~Hz}, \mathrm{OCH}_{2} \mathrm{CH}_{3}\right), 1.89(\mathrm{~m}, 2 \mathrm{H}$, $\left.\mathrm{CH}_{2} \mathrm{CH}\right), 2.05\left(\mathrm{~m}, 2 \mathrm{H}, \mathrm{CH}_{2} \mathrm{NCO}\right), 3.04\left(\mathrm{~m}, 2 \mathrm{H}, \mathrm{CH}_{2} \mathrm{NCO}\right), 3.08\left(\mathrm{~m}, 1 \mathrm{H}, \mathrm{CH}_{2} \mathrm{CH}\right), 4.19\left(\mathrm{~m}, 2 \mathrm{H}, \mathrm{CH}_{2} \mathrm{CH}_{2} \mathrm{NCO}\right), 4.37$ (q, 2H, J $7.12 \mathrm{~Hz} \mathrm{OCH}{ }_{2} \mathrm{CH}_{3}$ ), 5.35 (s, 2H, $\mathrm{CH}_{2} \mathrm{Ar}$ ), 6.58 (s, 1H, N-H), 6.85 (m, 1H, Ar-H), 7.06 (s, 1H, Ar-H), 7.21 (d, 1H, J $1.82 \mathrm{~Hz}, \mathrm{Ar}-\mathrm{H}), 7.38(\mathrm{~m}, 3 \mathrm{H}, \mathrm{Ar}-\mathrm{H}), 7.43$ (d, 2H, J $8.83 \mathrm{~Hz}, \mathrm{Ar}-\mathrm{H}), 7.69$ (d, 1H, J $8.56 \mathrm{~Hz}, \mathrm{Ar}-\mathrm{H}), 7.97$ (d, $2 \mathrm{H}$, J $8.77 \mathrm{~Hz}, \mathrm{Ar}-\mathrm{H}$; LC-MS (ESI+) $m / z=549[\mathrm{M}+\mathrm{H}]^{+}$.

$N$-(4-Acetylphenyl)-4-(6-chloro-1-(4-methoxybenzyl)-1H-benzo[d]imidazol-2-yl)piperidine-1-carboxamide $9\{4,2\}$

General Procedure G: $21\{4\}$ (0.097 g, $0.27 \mathrm{mmol})$, 4-acetylphenylisocyanate $(0.074 \mathrm{~g}, 0.46 \mathrm{mmol})$. Chromatography (50\% EtOAc, petrol), 9\{4,2\} $55 \%$; HPLC purity (as area \%) > 96; ${ }^{1} \mathrm{H} \mathrm{NMR}\left(400 \mathrm{MHz}, \mathrm{CDCl}_{3}\right.$ ) $1.82\left(\mathrm{~m}, 2 \mathrm{H}, \mathrm{CH}_{2} \mathrm{CH}_{2}-\mathrm{NH}\right), 2.05\left(\mathrm{~m}, 2 \mathrm{H}, \mathrm{CH}_{2} \mathrm{CH}_{2}-\mathrm{NH}\right), 2.48\left(\mathrm{~s}, 3 \mathrm{H}, \mathrm{Ar}-\mathrm{COCH}_{3}\right) 2.94\left(\mathrm{~m}, 3 \mathrm{H}, \mathrm{CH}_{2}-\mathrm{CH}_{2}-\mathrm{NH}\right.$ and CH$\mathrm{CH}_{2}$ ), $3.71(\mathrm{~s}, 3 \mathrm{H}, \mathrm{Ar}-\mathrm{OMe}), 4.12\left(\mathrm{~m}, \mathrm{br} 2 \mathrm{H}, \mathrm{CH}_{2}-\mathrm{N}\right), 5.22\left(\mathrm{~s}, 2 \mathrm{H}, \mathrm{CH}_{2}-\mathrm{N}\right), 6.62(\mathrm{~s}, 1 \mathrm{H}, \mathrm{N}-\mathrm{H}), 6.79(\mathrm{~d}, 2 \mathrm{H}, J$, 8.7, ArH), $6.84(\mathrm{~d}, 2 \mathrm{H}, J$ 8.7, Ar-H), $7.18(\mathrm{~m}, 2 \mathrm{H}, \mathrm{Ar}-\mathrm{H}), 7.39$ (d, 2H, J 8.8, Ar-H), 7.6 (dd, 1H, J 8.1, 1.04, Ar-H), 7.89 (d, $2 \mathrm{H}, J$ 8.8, Ar-H).; LC-MS (ESI+) $m / z 517[\mathrm{M}+\mathrm{H}]^{+}$.

RESULTS

Design

The search for MDM2-p53 inhibitors has revealed several classes of small molecules able to bind MDM2 at the p53 binding site and restore p53 activity.(21) Reflecting the hydrophobic nature of the MDM2-p53 interaction, all of these compounds are characterized by a central scaffold that directs hydrophobic substituents towards the three MDM2 sub-pockets, thus mimicking the key amino acid side-chains of p53. In an attempt to identify novel chemotypes acting as an anchor point for hydrophobic substituents, our attention was focused on compounds $\mathbf{1}$ and $\mathbf{7}$, taken as representative of two potent and structurally different classes of MDM2 inhibitors. The scaffold-hopping approach initially matched the two nitrogens of 
the Nutlin imidazoline ring 1 with the two nitrogens in the benzimidazole scaffold (Figure 2, red). Overlay of the Nutlin chlorophenyl rings with the benzimidazole gave two possible orientations of the chloro group in the scaffold ( 8 and 9 ). Positions 1 and 2 of the benzimidazole ring were used to append the hydrophobic piperidinyl amide substituents judged to be necessary for activity, resulting in two different substitution patterns, represented by general structures 8 and $\mathbf{9}$ (Figure 2).

$<$ figure 2>

Docking experiments showed two separate binding modes for the enantiomers of $8\{1,6\}$. In the case of the $(R)$-enantiomer the chlorobenzimidazole occupies the Phe19 pocket and the aryl urea fills the Leu26 pocket, whereas for the (S)-enantiomer the positions are reversed (Figures $3 a$ and $3 b$, respectively). The docked pose for $9\{1,6\}$ shows the arylurea occupying the Leu26 pocket and the benzyl group positioned within the Phe19 pocket (Figure 3c). Interestingly, the Trp23 subpocket is unoccupied for all of the binding modes generated. This pocket is known to be important from the published X-ray structures of small-molecule MDM2-p53 inhibitors. The other subpockets (Phe19 and Leu26) were occupied by the hydrophobic moieties of the ligands, thus rendering both libraries $\mathbf{8}$ and $\mathbf{9}$ worthy of exploration. For the alternative benzimidazoles $\mathbf{8}$, the $(S)$-enantiomers appeared to be more promising than $(R)$-enantiomers and therefore were prioritized for synthesis.

$<$ figure $3 a, b, c>$

Synthesis

The rapid synthesis of benzimidazole libraries has been described recently using a number of approaches, including solid-phase synthesis, $(43,44)$ and solution-phase methods. $(45-48)$ In this case, the required $N^{1}$ substitution was introduced via an $\mathrm{S}_{\mathrm{N}} \mathrm{Ar}$ reaction with an o-fluoronitrobenzene, followed by in-situ nitroreduction, using sodium dithionite under microwave heating, and cyclisation with the desired aldehyde to give the the appropriately substituted benzimidazoles 8 and 20.(49)

The synthesis of benzimidazoles 8 bearing substituted aryl groups required the use of suitably substituted arylacetaldehydes (10). These were prepared from the respective benzaldehydes using a Wittig reaction giving the methyl enol ethers (11), which were subjected to acidic hydrolysis (Scheme 1). The $S_{N} A r$ reaction of (S)-12 and 4-chloro-1-fluoro-2-nitrobenzene gave nitroaniline (13) in good yield. Reductive cyclisation of 13 with sodium dithionite in the presence of arylacetaldehydes 10 , under microwave heating, provided benzimidazoles 14\{1-4\} cleanly and in good yields. Deprotection with TFA gave piperidines 15\{1-4\} which were reacted in parallel with a series of isocyanates to give the final (S)-benzimidazoles 8\{1-4,1-10\} (Scheme 2). Single examples of the thiourea $\mathbf{1 6}$ and sulfonamide $\mathbf{1 7}$ derivatives were also prepared (Scheme 3 ). The synthesis of 2-(piperidin-4-yl)-benzimidazoles 9 required the Boc-protected isonipecotic aldehyde (18), prepared according to a literature procedure (Scheme 4).(50)

<scheme 1,2,3,4, Chemsets> 
Reaction of 4-chloro-1-fluoro-2-nitrobenzene with the required benzylamine under microwave heating gave nitroanilines (19). Reductive cyclisation of 19 with sodium dithionite in the presence of aldehydes 14, under microwave heating gave the benzimidazoles 20\{1-4\} in good yields. Deprotection with TFA gave piperidines 21 $\{1-4\}$ which were reacted in parallel with a series of isocyanates giving the final benzimidazoles $9\{1-4,1-10\}$ (Scheme 5).

<scheme 5>

\section{Biological evaluation}

We have previously demonstrated that the MDM2 and MDMX ELISA assays show good sensitivity over a wide range of $\mathrm{IC}_{50}$ values. In particular, compounds with low potency and poor predicted solubility have given reliable results. $(40,41)$ Compounds from libraries 8 and 9 were assayed against MDM2 and MDMX in parallel. The results are displayed in Tables 1 and 2. Overall, both series displayed limited MDM2 inhibition with only $\mathbf{8}\{4,7\}$, and $\mathbf{9}\{2,8\}$ showing sub-100 $\mu \mathrm{M}$ activity (MDM2 LE $=0.14$ and 0.15 , respectively). In contrast, $27 / 40$ examples of series 8 were sub-100 $\mu \mathrm{M}$ inhibitors of MDMX, and two examples showed sub$50 \mu \mathrm{M}$ activity, i.e. $\mathbf{8}\{3,4\}$ and $\mathbf{8}\{4,7\}$ (MDMX LE $=0.15$ ). Series $\mathbf{9}$ also had a greater number of sub-100 $\mu \mathrm{M}$ MDMX inhibitors (10/30), and four examples showed sub-50 $\mu \mathrm{M}$ activity, i.e. $\mathbf{9}\{2,2\}, \mathbf{9}\{2,3\}, \mathbf{9}\{2,8\}$ and $\mathbf{9}\{4,2\}$ (MDMX LE = $0.15-0.16$ ). Interestingly, the unsubstituted thiourea derivative 16 and the sulfonamide 17 showed improved MDMX inhibitory activity when compared with the equivalent urea $15\{1,6\}$.

$<$ table 1, 2>

<structure $8\{3,4\}, 8\{4,7\}, 9\{2,8\}, 9\{2,2\}, 9\{2,3\}, 9\{2,8\}, 9\{4,2\}>$

\section{DISCUSSION}

Recently, analysis of the contribution to binding of each portion of Nutlin-3 (1) has been reported, by the synthesis and analysis of fragments of the lead compound.(51) Systematic removal of the groups accessing each of the three sub-pockets of MDM2 allowed an assessment of their relative contribution to binding, and showed that removal of the 4-chlorophenyl group accessing the Trp23 pocket resulted in the most significant loss of activity. For example, 22 showed a $K_{d}$ for MDM2 of $1 \mathrm{mM}(L E=0.10)$. The activity of libraries $\mathbf{8}$ and $\mathbf{9}$ are consistent with the loss of potency associated with leaving the Trp23 sub-pocket vacant as predicted in the docked binding modes of both series. Interestingly, the results for MDMX suggest that the Trp23 sub-pocket may play a somewhat less significant role in the overall binding affinity, as libraries 8 and 9 showed improved activity.

$<$ structure22>

CONCLUSIONS 
Libraries of substituted benzimidazoles based on scaffold-hopping from structures $\mathbf{1}$ and $\mathbf{7}$ have been docked into MDM2 and evaluated. The efficient synthesis of two libraries ( $\mathbf{8}$ and $\mathbf{9}$ ) has been achieved by microwave-assisted reductive cyclisation. Both libraries were assayed for inhibition of the MDM2-p53 and MDMX-p53 protein-protein interaction. Members of each library showed sub-100 $\mu \mathrm{M}$ activity for MDM2 or MDMX and several mixed MDM2-MDMX inhibitors were identified with modest potency.

\section{SUPPORTING INFORMATION}

Synthetic and analytical details for compounds 8, 13, 14, 15, 16, 19, 20, 21.

\section{ACKNOWLEDGEMENTS}

This work was supported by Cancer Research UK and the European Union FP6 (DePPICT, grant number LSHCCT-2007-037834). Carlo Bawn and Karen Haggerty for expert technical assistance. Use of the EPSRC National Mass Spectrometry Service at the University of Wales (Swansea) is gratefully acknowledged.

\section{REFERENCES}

1. Toogood PL (2002) Inhibition of protein-protein association by small molecules: Approaches and progress. J Med Chem;45: 1543-58.

2. Yin H, Hamilton AD (2005) Strategies for targeting protein-protein interactions with synthetic agents. Angew Chem-Int Edit;44: 4130-63.

3. Wells JA, McClendon CL (2007) Reaching for high-hanging fruit in drug discovery at protein-protein interfaces. Nature;450: 1001-9.

4. $\quad$ Azzarito V, Long K, Murphy NS, Wilson AJ (2013) Inhibition of $\alpha$-helix-mediated protein-protein interactions using designed molecules. Nature Chemistry;5: 161-73.

5. Oliner JD, Kinzler KW, Meltzer PS, George DL, Vogelstein B (1992) Amplification of a Gene Encoding a P53-Associated Protein in Human Sarcomas. Nature;358: 80-3.

6. Shvarts A, Steegenga WT, Riteco N, van Laar T, Dekker P, Bazuine M, et al. (1996) MDMX: a novel p53-binding protein with some functional properties of MDM2. EMBO J;15: 5349-69.

7. Toledo F, Wahl GM (2006) Regulating the p53 pathway: in vitro hypotheses, in vivo veritas. Nat Rev Cancer;6: 909-23.

8. Gembarska A, Luciani F, Fedele C, Russell EA, Dewaele M, Villar S, et al. (2012) MDM4 is a key therapeutic target in cutaneous melanoma. Nat Med;18: 1239-47.

9. Momand J, Zambetti GP, Olson DC, George D, Levine A (1992) The $m d m-2$ oncogene product forms a complex with p53 protein and inhibits p53-mediated transactivation. Cell;69: 1237-45.

10. Fuchs SY, Adler V, Buschmann T, Wu XW, Ronai Z (1998) Mdm2 association with p53 targets its ubiquitination. Oncogene;17: 2543-7.

11. Haupt $Y$, Maya R, Kazaz A, Oren M (1997) Mdm2 promotes the rapid degradation of p53. Nature;387: 296-9.

12. Kubbutat MHG, Jones SN, Vousden KH (1997) Regulation of p53 stability by Mdm2. Nature;387: 299303.

13. Marine J-CW, Dyer MA, Jochemsen AG (2007) MDMX: from bench to bedside. J Cell Sci;120: 371-8.

14. Francoz S, Froment P, Bogaerts S, De Clercq S, Maetens M, Doumont G, et al. (2006) Mdm4 and Mdm2 cooperate to inhibit p53 activity in proliferating and quiescent cells in vivo. Proc Natl Acad Sci U S A;103: 3232-7.

15. Kussie PH, Gorina S, Marechal V, Elenbaas B, Moreau J, Levine AJ, et al. (1996) Structure of the MDM2 oncoprotein bound to the p53 tumor suppressor transactivation domain. Science;274: 94853. 
16. Weber $L$ (2010) Patented inhibitors of p53-Mdm2 interaction (2006 - 2008). Exp Opin Ther Patents;20: 179-91.

17. Khoury K, Popowicz GM, Holak TA, Domling A (2011) The p53-MDM2/MDMX axis - A chemotype perspective. MedChemComm;2: 246-60.

18. Vassilev LT, Vu BT, Graves B, Carvajal D, Podlaski F, Filipovic Z, et al. (2004) In Vivo Activation of the p53 Pathway by Small-Molecule Antagonists of MDM2. Science;303: 844-8.

19. Shangary S, Qin D, McEachern D, Liu M, Miller RS, Qiu S, et al. (2008) Temporal activation of p53 by a specific MDM2 inhibitor is selectively toxic to tumors and leads to complete tumor growth inhibition. Proc Nat Acad Sciences; 105: 3933-8.

20. Yu S, Qin D, Shangary S, Chen J, Wang G, Ding K, et al. (2009) Potent and Orally Active SmallMolecule Inhibitors of the MDM2-p53 Interaction. J Med Chem;52: 7970-3.

21. Popowicz GM, Dömling A, Holak TA (2011) The Structure-Based Design of Mdm2/Mdmx-p53 Inhibitors Gets Serious. Angewandte Chemie International Edition;50: 2680-8.

22. Mohammad RM, Wu J, Azmi AS, Aboukameel A, Sosin A, Wu S, et al. (2009) An MDM2 antagonist (MI-319) restores $\mathrm{p} 53$ functions and increases the life span of orally treated follicular lymphoma bearing animals. Mol Cancer;8:

23. Vu B, Wovkulich P, Pizzolato G, Lovey A, Ding Q, Jiang N, et al. (2013) Discovery of RG7112: A SmallMolecule MDM2 Inhibitor in Clinical Development. ACS Med Chem Lett;4: 466-9.

24. Ray-Coquard I, Blay J-Y, Italiano A, Le Cesne A, Penel N, Zhi J, et al. (2012) Effect of the MDM2 antagonist RG7112 on the P53 pathway in patients with MDM2-amplified, well-differentiated or dedifferentiated liposarcoma: an exploratory proof-of-mechanism study. The Lancet Oncology;13: 1133-40.

25. Ding Q, Zhang Z, Liu J-J, Jiang N, Zhang J, Ross TM, et al. (2013) Discovery of RG7388, a Potent and Selective p53-MDM2 Inhibitor in Clinical Development. J Med Chem;56: 5979-83.

26. Zhao Y, Liu L, Sun W, Lu J, McEachern D, Li X, et al. (2013) Diastereomeric Spirooxindoles as Highly Potent and Efficacious MDM2 Inhibitors. J Am Chem Soc;135: 7223-34.

27. Popowicz GM, Czarna A, Rothweiler U, Szwagierczak A, Krajewski M, Weber L, et al. (2007) Molecular Basis for the Inhibition of p53 by Mdmx. Cell Cycle;6: 2386-92.

28. Popowicz G, Czarna A, Holak T (2008) Structure of the human Mdmx protein bound to the p53 tumor suppressor transactivation domain. Cell Cycle;7: 2441-3.

29. Popowicz GM, Czarna A, Wolf S, Wang K, Wang W, Dömling A, et al. (2010) Structures of low molecular weight inhibitors bound to MDMX and MDM2 reveal new approaches for $\mathrm{p53-}$ MDMX/MDM2 antagonist drug discovery. Cell Cycle;9: 1104-11.

30. Macchiarulo A, Giacchè N, Carotti A, Baroni M, Cruciani G, Pellicciari R (2008) Targeting the Conformational Transitions of MDM2 and MDMX: Insights into Dissimilarities and Similarities of p53 Recognition. Journal of Chemical Information and Modeling;48: 1999-2009.

31. Kallen J, Goepfert A, Blechschmidt A, Izaac A, Geiser M, Tavares G, et al. (2009) Crystal Structures of Human MdmX ( $\mathrm{HdmX}$ ) in Complex with p53 Peptide Analogues Reveal Surprising Conformational Changes. J Biol Chem;284: 8812-21.

32. Joseph TL, Madhumalar A, Brown CJ, Lane DP, Verma CS (2010) Differential binding of p53 and nutlin to MDM2 and MDMX: Computational studies. Cell Cycle;9: 1167-81.

33. Reed D, Shen Y, Shelat AA, Arnold LA, Ferreira AM, Zhu F, et al. (2010) Identification and Characterization of the First Small Molecule Inhibitor of MDMX. J Biol Chem;285: 10786-96.

34. Graves B, Thompson T, Xia M, Janson C, Lukacs C, Deo D, et al. (2012) Activation of the p53 pathway by small-molecule-induced MDM2 and MDMX dimerization. Proc Nat Acad Sciences;109: 11788-93.

35. Schneider G, Neidhart W, Giller T, Schmid G (1999) "Scaffold-Hopping" by Topological Pharmacophore Search: A Contribution to Virtual Screening. Angewandte Chemie International Edition;38: 2894-6.

36. Cramer RD, Jilek RJ, Guessregen S, Clark SJ, Wendt B, Clark RD (2004) "Lead Hopping". Validation of Topomer Similarity as a Superior Predictor of Similar Biological Activities. J Med Chem;47: 6777-91.

37. Luk K-C, So S-S, Zhang J, Zhang Z. (2006) Oxindole Derivatives. 
38. Zhang Z, Ding Q, Liu J-J, Zhang J, Jiang N, Chu X-J, et al. (2014) Discovery of potent and selective spiroindolinone MDM2 inhibitor, RO8994, for cancer therapy. Bioorg Med Chem;22: 4001-9.

39. Evans BE, Rittle KE, Bock MG, DiPardo RM, Freidinger RM, Whitter WL, et al. (1988) Methods for drug discovery: development of potent, selective, orally effective cholecystokinin antagonists. $J$ Med Chem;31: 2235-46.

40. Hardcastle IR, Ahmed SU, Atkins H, Farnie G, Golding BT, Griffin RJ, et al. (2006) Small-molecule inhibitors of the MDM2-p53 protein-protein interaction based on an isoindolinone scaffold. J Med Chem;49: 6209-21.

41. Blackburn TJ, Ahmed S, Coxon CR, Liu JF, Lu XH, Golding BT, et al. (2013) Diaryl- and triaryl-pyrrole derivatives: inhibitors of the MDM2-p53 and MDMX-p53 protein-protein interactions.

Medchemcomm;4: 1297-304.

42. CDCC. (2005) GOLD. GOLD.

43. Bendale PM, Sun C-M (2002) Rapid Microwave-Assisted Liquid-Phase Combinatorial Synthesis of 2(Arylamino)benzimidazoles. J Comb Chem;4: 359-61.

44. Chen L-H, Chang C-M, Salunke DB, Sun C-M (2011) Divergent Synthesis of Unsymmetrical Annulated Biheterocyclic Compound Libraries: Benzimidazole Linked Indolo-benzodiazepines/quinoxaline. ACS Combinatorial Science;13: 391-8.

45. He Y, Wu B, Yang J, Robinson D, Risen L, Ranken R, et al. (2003) 2-Piperidin-4-yl-benzimidazoles with broad spectrum antibacterial activities. Bioorg Med Chem Lett;13: 3253-6.

46. Vourloumis D, Takahashi M, Simonsen KB, Ayida BK, Barluenga S, Winters GC, et al. (2003) Solidphase synthesis of benzimidazole libraries biased for RNA targets. Tetrahedron Lett;44: 2807-11.

47. Zou B, Yuan Q, Ma D (2007) Synthesis of 1,2-Disubstituted Benzimidazoles by a Cu-Catalyzed Cascade Aryl Amination/Condensation Process. Angewandte Chemie International Edition;46: 2598-601.

48. Micco I, Nencini A, Quinn J, Bothmann H, Ghiron C, Padova A, et al. (2008) Parallel synthesis of a series of potentially brain penetrant aminoalkyl benzoimidazoles. Bioorg Med Chem;16: 2313-28.

49. Yang D, Fokas D, Li J, Yu L, Baldino CM (2005) A Versatile Method for the Synthesis of Benzimidazoles from o-Nitroanilines and Aldehydes in One Step via a Reductive Cyclization. Synthesis;2005: 47-56.

50. Egbertson MS, Cook JJ, Bednar B, Prugh JD, Bednar RA, Gaul SL, et al. (1999) Non-Peptide GPIIb/IIla Inhibitors. 20. Centrally Constrained Thienothiophene $\alpha$-Sulfonamides Are Potent, Long Acting in Vivo Inhibitors of Platelet Aggregation. J Med Chem;42: 2409-21.

51. Fry DC, Wartchow C, Graves B, Janson C, Lukacs C, Kammlott U, et al. (2013) Deconstruction of a Nutlin: Dissecting the Binding Determinants of a Potent Protein-Protein Interaction Inhibitor. ACS Med Chem Lett; 4: 660-5. 


\section{TABLE CAPTIONS}

Table 1. MDM2-p53 and MDMX-p53 inhibitory activity of compound libraries $\mathbf{1 3}$ and $\mathbf{8 .}$

Table 2. MDM2-p53 and MDMX-p53 inhibitory activity of compound libraries 20 and $\mathbf{9 .}$

\section{FIGURE CAPTIONS}

Chart 1: MDM2-p53 clinical trials candidates with disclosed structures.

Figure 1: Structure of the p53-MDM2 complex (1RV1) (MDM2 white, p53 peptide yellow).(15)

Figure 2: Schematic for scaffold hopping and docking approach.

Figure 3: Putative binding modes for compounds: a) $8(R)$-enantiomers; b) 8 (S)-enantiomers; c) 9.

\section{SCHEME CAPTIONS}

Scheme 1: Synthesis of substituted phenylacetaldehydes $10 .^{a}$

${ }^{a}$ Reagents and Conditions: a) $\mathrm{MeOCH}_{2} \mathrm{PPh}_{3} \mathrm{Br}$, KOt-Bu, THF, rt; b) $\mathrm{HCO}_{2} \mathrm{H}, \mathrm{DCM}$, rt.

Scheme 2: Synthesis of benzimidazole library $8 .^{2}$

a Reagents and Conditions: a) DMF, $\mathrm{Na}_{2} \mathrm{CO}_{3}, 70^{\circ} \mathrm{C}$; b) $\mathrm{ArCH}_{2} \mathrm{CHO}, 13, \mathrm{Na}_{2} \mathrm{~S}_{2} \mathrm{O}_{4}, \mathrm{MeOH}, \mathrm{H}_{2} \mathrm{O}, \mathrm{MW}, 100{ }^{\circ} \mathrm{C}$; c)i) TFA, DCM, rt; ii) $\mathrm{K}_{2} \mathrm{CO}_{3}, \mathrm{MeOH}, \mathrm{rt}$; d) R'NCO, DCM, rt.

Scheme 3: Synthesis of benzimidazoles 16 and $17 .{ }^{a}$

a Reagents and Conditions: a) $\mathrm{R}^{\prime} \mathrm{NCS}, \mathrm{DCM}, \mathrm{rt}$; b) $\mathrm{PhCH}_{2} \mathrm{SO}_{2} \mathrm{Cl}, \mathrm{Et}{ }_{3} \mathrm{~N}, \mathrm{DCM}$, rt.

Scheme 4: Synthesis of piperidine 1 18. $^{\text {a }}$

${ }^{a}$ Reagents and Conditions: a) $\mathrm{Boc}_{2} \mathrm{O}, \mathrm{NaOH}$, dioxane, rt; b) $\mathrm{BH}_{3}-\mathrm{THF}, \mathrm{THF}, \mathrm{rt}$; c) PCC, NaOAc, $4 \AA \AA$ molecular sieves, DCM, rt.

Scheme 5: Synthesis of benzimidazole library $9 .^{\text {a }}$

${ }^{\text {a }}$ Reagents and Conditions: a) $\mathrm{ArNH}_{2}, \mathrm{~K}_{2} \mathrm{CO}_{3}$, EtOH, rt; b) 19, $\mathrm{Na}_{2} \mathrm{~S}_{2} \mathrm{O}_{4}, \mathrm{MeOH}, \mathrm{H}_{2} \mathrm{O}, \mathrm{MW}, 100{ }^{\circ} \mathrm{C}$; c) i)TFA, DCM, $\mathrm{rt}$; ii) $\mathrm{K}_{2} \mathrm{CO}_{3}, \mathrm{rt}$; d) $\mathrm{R}^{\prime} \mathrm{NCO}, \mathrm{DCM}, \mathrm{rt}$. 
Table 1.

<structure 8>

\begin{tabular}{|c|c|c|c|c|c|}
\hline Compound & $\begin{array}{c}\text { MDM2 IC } 50 \\
(\mu \mathrm{M})\end{array}$ & $\begin{array}{c}\text { MDMX IC } 50 \\
(\mu \mathrm{M})\end{array}$ & Compound & $\begin{array}{c}\text { MDM2 IC } 50 \\
(\mu \mathrm{M})\end{array}$ & $\begin{array}{l}\text { MDMX IC } \\
(\mu \mathrm{M})\end{array}$ \\
\hline $13\{1\}$ & $>500$ & 248 & $8\{3,1\}$ & 170 & $64 \pm 20$ \\
\hline $13\{2\}$ & $290 \pm 38$ & $104 \pm 23$ & $8\{3,2\}$ & - & - \\
\hline $13\{3\}$ & $>500$ & $>500$ & $8\{3,3\}$ & $121 \pm 40$ & $92 \pm 18$ \\
\hline $13\{4\}$ & 211 & 130 & $8\{3,4\}$ & $120 \pm 37$ & $43 \pm 15$ \\
\hline $8\{1,1\}$ & $183 \pm 20$ & $70 \pm 19$ & $8\{3,5\}$ & $213 \pm 158$ & $74 \pm 12$ \\
\hline $8\{1,2\}$ & $208 \pm 33$ & $105 \pm 54$ & $8\{3,6\}$ & 191 & 187 \\
\hline $8\{1,3\}$ & $202 \pm 24$ & $54 \pm 14$ & $8\{3,7\}$ & $146 \pm 63$ & $77 \pm 20$ \\
\hline $8\{1,4\}$ & 176 & 117 & $8\{3,8\}$ & $>500$ & 116 \\
\hline $8\{1,5\}$ & $202 \pm 51$ & $92 \pm 51$ & $8\{3,9\}$ & $257 \pm 146$ & $131 \pm 48$ \\
\hline $8\{1,6\}$ & 218 & 100 & $8\{3,10\}$ & 177 & 100 \\
\hline $8\{1,7\}$ & $203 \pm 80$ & $61 \pm 30$ & $8\{4,1\}$ & - & - \\
\hline $8\{1,8\}$ & $196 \pm 61$ & $69 \pm 5$ & $8\{4,2\}$ & $201 \pm 96$ & $95 \pm 13$ \\
\hline $8\{1,9\}$ & $>500$ & 156 & $8\{4,3\}$ & 115 & 207 \\
\hline $8\{1,10\}$ & 257 & 169 & $8\{4,4\}$ & $166 \pm 55$ & $78 \pm 16$ \\
\hline $8\{2,1\}$ & 151 & 86 & $8\{4,5\}$ & $188 \pm 102$ & $173 \pm 121$ \\
\hline $8\{2,2\}$ & $134 \pm 86$ & $81 \pm 33$ & $8\{4,6\}$ & $188 \pm 33$ & $109 \pm 39$ \\
\hline $8\{2,3\}$ & $148 \pm 61$ & $60 \pm 10$ & $8\{4,7\}$ & $72 \pm 20$ & $43 \pm 24$ \\
\hline $8\{2,4\}$ & $201 \pm 128$ & $154 \pm 79$ & $8\{4,8\}$ & $202 \pm 157$ & $76 \pm 24$ \\
\hline $8\{2,5\}$ & $114 \pm 38$ & $80 \pm 13$ & $8\{4,9\}$ & $198 \pm 75$ & $99 \pm 26$ \\
\hline $8\{2,6\}$ & $125 \pm 35$ & $83 \pm 29$ & $8\{4,10\}$ & $131 \pm 45$ & $64 \pm 13$ \\
\hline $8\{2,7\}$ & $154 \pm 81$ & $57 \pm 14$ & 16 & $144 \pm 44$ & $49 \pm 14$ \\
\hline $8\{2,8\}$ & $153 \pm 112$ & $50 \pm 6$ & 17 & $212 \pm 1$ & $59 \pm 36$ \\
\hline $8\{2,9\}$ & $165 \pm 30$ & $73 \pm 14$ & & & \\
\hline $8\{2,10\}$ & $103 \pm 36$ & $63 \pm 35$ & & & \\
\hline
\end{tabular}


Table 2.

<structure 9>

\begin{tabular}{|c|c|c|c|c|c|}
\hline Compound & $\begin{array}{c}\text { MDM2 IC } 50 \\
(\mu \mathrm{M})\end{array}$ & $\begin{array}{c}\text { MDMX IC } 50 \\
(\mu \mathrm{M})\end{array}$ & Compound & $\begin{array}{c}\text { MDM2 IC } 50 \\
(\mu \mathrm{M})\end{array}$ & $\begin{array}{c}\text { MDMX IC } \\
(\mu \mathrm{M})\end{array}$ \\
\hline $20\{1\}$ & 253 & $>500$ & $9\{2,9\}$ & - & - \\
\hline $20\{2\}$ & 248 & 217 & $9\{2,10\}$ & 212 & 101 \\
\hline $20\{3\}$ & $>500$ & $>500$ & $9\{3,1\}$ & - & - \\
\hline $20\{4\}$ & $>500$ & $>500$ & $9\{3,2\}$ & 201 & 103 \\
\hline $21\{1\}$ & 282 & $>500$ & $\mathbf{9}\{3,3\}$ & $182 \pm 21$ & $81 \pm 20$ \\
\hline $\mathbf{9}\{1,1\}$ & - & - & $9\{3,4\}$ & $291 \pm 117$ & $252 \pm 136$ \\
\hline $\mathbf{9}\{1,2\}$ & $307 \pm 132$ & $154 \pm 70$ & $9\{3,5\}$ & 183 & $>500$ \\
\hline $\mathbf{9}\{1,3\}$ & $256 \pm 51$ & $94 \pm 37$ & $9\{3,6\}$ & $269 \pm 39$ & $200 \pm 100$ \\
\hline $\mathbf{9}\{1,4\}$ & $141 \pm 22$ & $98 \pm 62$ & $9\{3,7\}$ & $>500$ & $>500$ \\
\hline $\mathbf{9}\{1,5\}$ & $>500$ & 151 & $\mathbf{9}\{3,8\}$ & $149 \pm 61$ & $94 \pm 38$ \\
\hline $\mathbf{9}\{1,6\}$ & $>500$ & 231 & $9\{3,9\}$ & 218 & 123 \\
\hline $\mathbf{9}\{1,7\}$ & $>500$ & 194 & $\mathbf{9}\{3,10\}$ & 218 & 201 \\
\hline $\mathbf{9}\{1,8\}$ & 151 & 194 & $9\{4,1\}$ & $>500$ & $>500$ \\
\hline $\mathbf{9}\{1,9\}$ & $>500$ & $>500$ & $9\{4,2\}$ & $314 \pm 65$ & $49 \pm 22$ \\
\hline $\mathbf{9}\{1,10\}$ & $204 \pm 69$ & $111 \pm 63$ & $\mathbf{9}\{4,3\}$ & $>500$ & 145 \\
\hline $\mathbf{9}\{2,1\}$ & - & - & $9\{4,4\}$ & - & - \\
\hline $\mathbf{9}\{2,2\}$ & $130 \pm 65$ & $46 \pm 11$ & $9\{4,5\}$ & - & - \\
\hline $\mathbf{9}\{2,3\}$ & 243 & 45 & $9\{4,6\}$ & $>500$ & 245 \\
\hline $\mathbf{9}\{2,4\}$ & $156 \pm 40$ & $113 \pm 74$ & $9\{4,7\}$ & - & - \\
\hline $9\{2,5\}$ & - & - & $\mathbf{9}\{4,8\}$ & $180 \pm 15$ & $73 \pm 16$ \\
\hline $\mathbf{9}\{2,6\}$ & - & - & $\mathbf{9}\{4,9\}$ & - & - \\
\hline $\mathbf{9}\{2,7\}$ & $161 \pm 68$ & $68 \pm 29$ & $\mathbf{9}\{4,10\}$ & $>500$ & 184 \\
\hline $9\{2,8\}$ & $88 \pm 43$ & $31 \pm 10$ & & & \\
\hline
\end{tabular}




\section{Searching for dual inhibitors of the MDM2-p53 and MDMX-p53 protein-protein interaction by a scaffold-hopping approach}

Andrey Zaytsev, Barry Dodd, Matteo Magnani, Chiara Ghiron, Bernard T. Golding, Roger J. Griffin, Junfeng Liu, Xiaohong Lu, Iolanda Micco, David R. Newell, Alessandro Padova, Graeme Robertson, John Lunec, Ian R. Hardcastle*

\section{CHEMSETS}

Chemsets

$\operatorname{Ar}=$<smiles>CCc1ccccc1</smiles>

$\{1\}$<smiles>CC(C)(C)c1cccc(Cl)c1</smiles>

$\{2\}$<smiles>CCc1cccc(OC)c1</smiles>

$\{3\}$<smiles>CCc1ccc(OC)cc1</smiles>

$\{4\}$<smiles>[R]=CI1C=CC([N+](=O)[O-])=CC1</smiles>

$\{1\}$<smiles>ICc1ccccc1</smiles>

$\{6\}$<smiles>CC(=O)c1ccc(I)cc1</smiles>

\{2\}<smiles>CCOC(=O)c1cccc(I)c1</smiles>

\{7\}<smiles>COc1cc(I)cc(OC)c1OC</smiles>

\{3\}<smiles>CCOC(=O)c1ccc(I)cc1</smiles>

$\{8\}$<smiles>FC(F)(F)Oc1ccc(I)cc1</smiles>

$\{4\}$<smiles>CCc1ccc(F)cc1</smiles>

$\{9\}$<smiles>FC(F)(F)c1ccc(I)cc1</smiles>

$\{5\}$

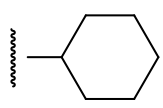

$\{10\}$

\section{SYNTHESIS}

\section{General Methods}

Reagents were purchased from fine chemicals vendors, and used as received unless otherwise stated. Solvents were purified and stored according to standard procedures. Petrol refers to that fraction in the boiling range $40-60{ }^{\circ} \mathrm{C}$. THF refers to anhydrous tetrahydrofuran from commercial sources. Microwave reactions were performed using a Biotage Initiator instrument in sealed glass tubes. Melting points were obtained on a Stuart Scientific SMP3 apparatus and are uncorrected. Thin layer chromatography was performed using silica gel plates (Kieselgel 60F254; 0.2 mm), and visualized with UV light or potassium permanganate. Chromatography was conducted under medium pressure in glass columns or using a Biotage SP4 instrument in prepacked columns (FLASH+ Silica columns (40-63 $\mu \mathrm{m}, 60 \AA ̊$ ). Proton $\left({ }^{1} \mathrm{H}\right)$ and carbon $\left({ }^{13} \mathrm{C}\right)$ nuclear magnetic resonance (NMR) spectra were recorded on a Bruker Spectrospin $A C$ $300 \mathrm{E}\left({ }^{1} \mathrm{H}\right.$ at $300 \mathrm{MHz},{ }^{13} \mathrm{C}$ at $\left.75 \mathrm{MHz}\right)$, a Jeol JNM-LA500 spectrometer $\left({ }^{1} \mathrm{H}\right.$ at $500 \mathrm{MHz},{ }^{13} \mathrm{C}$ at $\left.125 \mathrm{MHz}\right)$, or a Bruker Avance II $500\left({ }^{1} \mathrm{H}\right.$ at $500 \mathrm{MHz},{ }^{13} \mathrm{C}$ at $\left.125 \mathrm{MHz}\right)$ employing the solvent as internal standard. IR spectra were recorded on a Bio-Rad FTS 3000MX diamond ATR. Liquid Chromatography-Mass Spectrometry (LCMS) was carried out on a Micromass Platform instrument operating in positive and negative ion electrospray mode, employing a $50 \times 4.6 \mathrm{~mm}$ C18 column (Waters Symmetry or Waters Atlantis) 5 or 12 min gradient elution with $0.05 \%$ formic acid in methanol 
(10-90\%). Accurate masses were measured using a Finnigan MAR 95 XP or a Finnigan MAR 900 XLT at the EPSRC National Mass Spectrometry Service Centre (Chemistry Department, University of Wales, Swansea, Wales, SA2 8PP). tert-Butyl 3-((4-chloro-2-nitrophenyl)amino)piperidine-1-carboxylate (13)

1,1-Dimethylethyl-3-aminopiperidine-1-carboxylate $(1.29 \mathrm{~g}, 6.44 \mathrm{mmol})$ was added to a suspension of $\mathrm{Na}_{2} \mathrm{CO}_{3}(0.819$ $\mathrm{g}, 7.73 \mathrm{mmol})$ and 4-chloro-1-fluoro-2-nitrobenzene $(1.13 \mathrm{~g}, 6.44 \mathrm{mmol})$ in dry DMF $(20 \mathrm{~mL})$, and stirred at $70{ }^{\circ} \mathrm{C}$ for 2.5 hours. The mixture was diluted with water $(200 \mathrm{~mL})$ and extracted with ethyl acetate $(3 \times 70 \mathrm{~mL})$. The combined organic layers were washed with water $(2 \times 50 \mathrm{~mL})$, brine $(50 \mathrm{~mL})$, and dried $\left(\mathrm{MgSO}_{4}\right)$, and evaporated. Chromatography (silica; 0-20\% ethyl acetate, petrol) gave 13 as a bright orange oil $(1.80 \mathrm{~g}, 79 \%) .{ }^{1} \mathrm{H}$ NMR (400 MHz, $\left.\mathrm{CD}_{3} \mathrm{CN}\right) \delta 1.34$ (br s, 9H), 1.51-1.62 (1H, br m), $1.64-1.83(2 \mathrm{H}, \mathrm{br} \mathrm{m}), 1.92-1.99(1 \mathrm{H}, \mathrm{m}), 3.27-3.53(3 \mathrm{H}, \mathrm{br} \mathrm{m}), 3.54$ - $3.66(1 \mathrm{H}, \mathrm{br} \mathrm{m}), 3.69-3.76(1 \mathrm{H}, \mathrm{m}), 7.05(1 \mathrm{H}, \mathrm{d}, J$ 9.3, $\mathrm{Ar}), 7.47(1 \mathrm{H}, \mathrm{dd}, J 2.5$ and 9.3, $\mathrm{Ar}), 8.07(1 \mathrm{H}, \mathrm{d}, J 6.7, \mathrm{NH})$, $8.11(1 \mathrm{H}, \mathrm{d}, \mathrm{J} 2.5, \mathrm{Ar}) ;{ }^{13} \mathrm{C}$ NMR $\left(125 \mathrm{MHz}, \mathrm{CD}_{3} \mathrm{CN}\right)$ 23.0, 28.4, 30.0, 44.3, 45.3, 48.5, 48.9, 80.2, 117.5, 120.4, 126.5, 132.9, 137.2, 144.4, 155.4; $\mathrm{HRMS} \mathrm{C}{ }_{16} \mathrm{H}_{26} \mathrm{Cl}_{2} \mathrm{O}_{4} \mathrm{~N}_{4} \mathrm{Cl}\left[\mathrm{M}+\mathrm{NH}_{4}\right]^{+}$calcd 373.1637, obsd 373.1642.

\section{Chemical Synthesis.}

\section{General Procedure A - Synthesis of compounds (14).}

A mixture of the appropriate aldehyde (1.2 eq.), sodium dithionite ( $86 \%$ purity; $1.98 \mathrm{~g}, 9.78 \mathrm{mmol}, 3$ eq.) and 13 $(1.16 \mathrm{~g}, 3.26 \mathrm{mmol}, 1.0 \mathrm{eq}$.) in methanol $(12 \mathrm{~mL})$ and water $(3 \mathrm{~mL})$ was heated in a microwave reactor for 10 minutes at $100{ }^{\circ} \mathrm{C}$. The sample was diluted with ethyl acetate $(20 \mathrm{~mL})$ and washed with water $(20 \mathrm{~mL})$ and brine $(20 \mathrm{~mL})$. The organic layer was dried $\left(\mathrm{MgSO}_{4}\right)$, and evaporated. Chromatography (silica; ethyl acetate, petrol) gave compound 14 .

(S)-tert-Butyl 3-(2-benzyl-5-chloro-1H-benzo[d]imidazol-1-yl)piperidine-1-carboxylate 14\{1\}.

General Procedure A: phenylacetaldehyde $(0.42 \mathrm{~mL}, 3.59 \mathrm{mmol}) .14\{1\}(0.94 \mathrm{~g}, 61 \%)$, pale pink solid; HPLC purity (as area \%) > 99; ${ }^{1} \mathrm{H}$ NMR $\left(400 \mathrm{MHz}, \mathrm{CDCl}_{3}\right) \delta 1.43$ (br.s., 12H), 1.71(1H, d, J 12.4), $2.09(1 \mathrm{H}, \mathrm{qd}, J 3.7$ and 12.8), $2.65(1 \mathrm{H}$, app.), 3.29 (1H, app.t), 4.13-4.21 (2H, m), $4.25(1 \mathrm{H}, \mathrm{d}, J$ 15.6), 4.41 (1H, br. d, J 15.6), $7.16(1 \mathrm{H}, \mathrm{dd}, J 1.8$ and 8.7, Ar), 7.18-7.30 (5H, m, Ar), $7.34\left(1 \mathrm{H}, \mathrm{d}, J\right.$ 8.7, Ar), $7.74\left(1 \mathrm{H}, \mathrm{d}, J\right.$ 1.8, Ar); ${ }^{13} \mathrm{C}$ NMR (125 MHz, CDCl $)$ 24.9, 28.4, 28.8, 35.3, 43.6, 46.8, 53.7, 80.4, 112.4, 119.9, 122.6, 127.3, 127.6, 128.4, 129.0, 132.4, 136.1, 144.3, 154.4, 154.7.

(S)- tert-Butyl-3-(5-chloro-2-(3-chlorobenzyl)-1H-benzo[d]imidazol-1-yl)piperidine-1-carboxylate 14\{2\}.

General Procedure A: 2-(3-chlorophenyl) acetaldehyde (0.604 g, $3.91 \mathrm{mmol}) .14\{2\}$ (0.76 g, 51\%), off-white solid; mp 64-65 ${ }^{\circ} \mathrm{C}$; HPLC purity (as area \%) > 97; IR $\left(\mathrm{cm}^{-1}\right)$ 2974, 2928, 2872, 1686, 1510, 1467, 1453, 1435, 1414, 1404, 1391, 1365, 1256, 1238, 1142, 1063, 994, 910, 884, 857, 792, 769, 684; ${ }^{1} \mathrm{H}$ NMR $\left(500 \mathrm{MHz}, \mathrm{CDCl}_{3}\right) \delta 1.38$ (br.s., $\left.11 \mathrm{H}\right), 1.70$ (2H, app.d), $2.09(1 \mathrm{H}, \mathrm{qd}, J 4.0$ and 12.8), $2.63(1 \mathrm{H}, \mathrm{app} . \mathrm{t}), 3.25(1 \mathrm{H}, \mathrm{br} \mathrm{s}), 4.04-4.11(2 \mathrm{H}, \mathrm{m}), 4.17(1 \mathrm{H}, \mathrm{d}, J 16.0), 4.32$ $(1 \mathrm{H}$, br. d, J 16.0), 7.00-7.02 (1H, m, Ar), $7.12(1 \mathrm{H}, \mathrm{dd}, J 1.9$ and 8.7, Ar), 7.15-7.17 (3H, m, Ar), $7.30(1 \mathrm{H}, \mathrm{d}, J$ 8.7, Ar), $7.68(1 \mathrm{H}, \mathrm{d}, J \mathrm{~J} .9, \mathrm{Ar}) ;{ }^{13} \mathrm{C} \mathrm{NMR}\left(125 \mathrm{MHz}, \mathrm{CDCl}_{3}\right)$ 25.0, 28.4, 28.9, 34.7, 43.6, 46.8, 53.7, 80.4, 112.4, 120.0, 122.8, $126.5,127.5,127.7,128.4,130.2,132.3,134.9,138.1,144.2,153.5 ; \mathrm{HRMS} \mathrm{C}_{24} \mathrm{H}_{27} \mathrm{Cl}_{2} \mathrm{~N}_{3} \mathrm{O}_{2}[\mathrm{M}+\mathrm{H}]^{+}$calcd 460.1553 , obsd 460.1552 .

(S)-tert-Butyl-3-(5-chloro-2-(3-methoxybenzyl)-1H-benzo[d]imidazol-1-yl)piperidine-1-carboxylate 14\{3\}.

General Procedure A: 2-(3-methoxyphenyl) acetaldehyde $(0.588 \mathrm{~g}, 3.91 \mathrm{mmol})$ gave $\mathbf{1 4}\{3\}(0.66 \mathrm{~g}, 44 \%)$ as a pinkish solid; mp 62-63 ${ }^{\circ} \mathrm{C}$; HPLC purity (as area \%) > 99; IR $\left(\mathrm{cm}^{-1}\right)$ 3000, 2973, 2931, 2862, 1686, 1599, 1585, 1490, 1454, $1435,1404,1365,1256,1283,1143,1044,908,881,855,794,768 ;{ }^{1} \mathrm{H} N M R\left(500 \mathrm{MHz}, \mathrm{CDCl}_{3}\right) \delta 1.43$ (br.s., 11H), 1.71(2H, app.d), $2.05(1 \mathrm{H}, \mathrm{qd}, J 3.6$ and 12.6$), 2.60(1 \mathrm{H}, \mathrm{app} . \mathrm{t}), 3.25(1 \mathrm{H}, \mathrm{br} \mathrm{s}), 3.66\left(3 \mathrm{H}, \mathrm{s}, \mathrm{OCH}_{3}\right), 4.11-4.17(3 \mathrm{H}, \mathrm{m})$, 
4.16 (1H, d, J 15.8), $4.32(1 \mathrm{H}, \mathrm{br} . \mathrm{d}, J$ 15.8), 6.67-6.71 (3H, m, Ar), 7.09 (1H, dd, J 1.9 and 8.7, Ar), 7.12-7.15 (1H, m, Ar), 7.29 (1H, d, J 8.7, Ar), $7.67(1 \mathrm{H}, \mathrm{d}, J$ 1.9, $\mathrm{Ar}) ;{ }^{13} \mathrm{C} \mathrm{NMR}\left(125 \mathrm{MHz}, \mathrm{CDCl}_{3}\right)$ 25.0, 28.4, 28.8, 35.2, 43.6, 46.8, 53.6, $55.2,80.3,112.3,112.7,114.0,119.9,120.6,122.5,127.6,129.9,132.3,137.6,144.2,154.2,154.7,160.0 ;$ HRMS $\mathrm{C}_{25} \mathrm{H}_{30}{ }^{35} \mathrm{ClN}_{3} \mathrm{O}_{3}[\mathrm{M}+\mathrm{H}]^{+}$calcd 456.2048, obsd 456.2053.

\section{(S)- tert-Butyl 3-(5-chloro-2-(4-methoxybenzyl)-1H-benzo[d]imidazol-1-yl)piperidine-1-carboxylate 14\{4\}.}

General Procedure A: 2-(4-methoxyphenyl) acetaldehyde (0.588 g, $3.91 \mathrm{mmol})$. 14\{4\} (0.40 g, 27\%), off-white solid; $\mathrm{mp}$ 60-61 ${ }^{\circ} \mathrm{C}$; HPLC purity (as area \%) > 99; IR $\left(\mathrm{cm}^{-1}\right)$ 2969, 2933, 2868, 2836, 1689, 1612, 1511, 1454, 1418, 1404, $1389,1366,1303,1242,1176,1142,1107,1063,1032,923,901,885,857,819,793,775,765,733 ;{ }^{1} H$ NMR $\delta(500$ $\left.\mathrm{MHz}, \mathrm{CDCl}_{3}\right) \delta 1.47$ (br.s., $\left.11 \mathrm{H}\right), 1.77(2 \mathrm{H}$, app.d), $2.14(1 \mathrm{H}, \mathrm{qd}, J 3.6$ and 12.7), $2.70(1 \mathrm{H}$, app.t), 3.33 (1H, br s), 3.79 $\left(3 \mathrm{H}, \mathrm{s}, \mathrm{OCH}_{3}\right), 4.10-4.25(3 \mathrm{H}, \mathrm{m}), 4.36(1 \mathrm{H}, \mathrm{br} . \mathrm{d}, J 15.7), 6.84(2 \mathrm{H}, \mathrm{d}, J$ 8.7, Ar), $7.13(1 \mathrm{H}, \mathrm{d}, J$ 8.7, Ar), $7.19(1 \mathrm{H}, \mathrm{dd}, J$ 1.9 and 8.7, $\mathrm{Ar}), 7.38(1 \mathrm{H}, \mathrm{d}, J$ 8.7, $\mathrm{Ar}), 7.67(1 \mathrm{H}, \mathrm{d}, J$ 1.9, $\mathrm{Ar}) ;{ }^{13} \mathrm{C} \mathrm{NMR}\left(125 \mathrm{MHz}, \mathrm{CDCl}_{3}\right)$ 25.0, 28.3, 28.8, 34.3, 43.6, $46.8,53.6,55.2,80.3,112.4,114.4,119.8,122.5,127.5,128.0,129.3,132.4,144.2,154.7,158.7 ; \mathrm{HRMS} \mathrm{C}_{25} \mathrm{H}_{30} \mathrm{ClN}_{3} \mathrm{O}_{3}$ $[\mathrm{M}+\mathrm{H}]^{+}$calcd 456.2048, obsd 456.2046 .

General Procedure B. Compound 14 was dissolved in DCM $(2 \mathrm{~mL})$ and TFA $(3 \mathrm{~mL})$ and the mixture stirred at rt for 1 hour, then evaporated. The residue was diluted with DCM $(20 \mathrm{~mL})$ and neutralised with sodium carbonate (sat.; 20 $\mathrm{mL})$. The organic layer was dried $\left(\mathrm{MgSO}_{4}\right)$ to give $\mathbf{1 5}$ which was used without further purification.

(S)-2-Benzyl-5-chloro-1-(piperidin-3-yl)-1H-benzo[d]imidazole 15\{1\}.

General Procedure B: $14\{1\}$ (0.725 g, 1.575 mmol). 15\{1\} (0.53 g, 94\%), white solid; ${ }^{1} \mathrm{H}$ NMR (500 MHz, $\left.\mathrm{CDCl}_{3}\right) 1.42$ $(1 \mathrm{H}, \mathrm{qt}, J 4.0$ and 12.9), $1.54(1 \mathrm{H}$, app.d), $1.67(1 \mathrm{H}, \mathrm{br} \mathrm{s}, \mathrm{NH}), 1.73-1.77(1 \mathrm{H}, \mathrm{m}), 2.12(1 \mathrm{H}, \mathrm{qd}, J 4.2$ and 12.8$), 2.57$ $(1 \mathrm{H}, \mathrm{td}, J=2.5$ and 12.4), $2.79(1 \mathrm{H}, \mathrm{dd}, J 3.7$ and 11.4), 3.02(1H, br d), $3.23(1 \mathrm{H}, \mathrm{t}, J 11.4), 4.20-4.26(1 \mathrm{H}, \mathrm{m}), 4.31(1 \mathrm{H}$, d, J 15.7, -CHHAr), 4.37 (1H, d, J 15.7, -CHHAr), 7.16 (1H, dd, J 2.0 and 8.6, Ar), 7.21-7.31 (5H, m, Ar), $7.42(1 \mathrm{H}, \mathrm{d}, J$ 8.6, $\mathrm{Ar})$; $7.73(1 \mathrm{H}, \mathrm{d}, \mathrm{J} 2.0, \mathrm{Ar}) ;{ }^{13} \mathrm{C}$ NMR $\left(125 \mathrm{MHz}, \mathrm{CDCl}_{3}\right)$ 26.5, 29.2, 35.2, 45.9, 49.6, 55.3, 112.6, 119.6, 122.3, 127.1, $127.3,128.3,128.8,132.5,136.2,144.2,154.4$;

\section{(S)-5-Chloro-2-(3-chlorobenzyl)-1-(piperidin-3-yl)-1H-benzo[d]imidazole 15\{2\}.}

General Procedure B: $14\{2\}$ (0.725 g, 1.575 mmol). $15\{2\}$ (0.53 g, 94\%), white solid; ${ }^{1} \mathrm{H}$ NMR (500 MHz, $\left.\mathrm{CDCl}_{3}\right) 1.46$ $(1 \mathrm{H}, \mathrm{qt}, J 4.0$ and 12.9), $1.57(1 \mathrm{H}$, app.d), $1.75(1 \mathrm{H}, \mathrm{br} \mathrm{s}, \mathrm{NH}), 1.76-1.81(1 \mathrm{H}, \mathrm{m}), 2.14(1 \mathrm{H}, \mathrm{qd}, J 4.1$ and 12.7), $2.59(1 \mathrm{H}$, $\mathrm{td}, J 2.6$ and 12.4), $2.88(1 \mathrm{H}, \mathrm{dd}, J 3.6$ and 11.5), 3.04-3.06 (1H, m), $3.27(1 \mathrm{H}, \mathrm{t}, J 11.5), 4.17-4.23(1 \mathrm{H}, \mathrm{m}), 4.27(1 \mathrm{H}, \mathrm{d}$, J 16.0, -CHHAr), $4.35(1 \mathrm{H}, \mathrm{d}, J$ 16.0, -CHHAr), 7.09-7.12 (1H, m, Ar), $7.17(1 \mathrm{H}, \mathrm{dd}, J$ 2.0 and 8.7, Ar), 7.22-7.23 (3H, m, Ar), $7.44(1 \mathrm{H}, \mathrm{d}, J$ 8.7, $\mathrm{Ar}) ; 7.73(1 \mathrm{H}, \mathrm{d}, J$ 2.0, $\mathrm{Ar}) ;{ }^{13} \mathrm{C} \mathrm{NMR}\left(125 \mathrm{MHz}, \mathrm{CDCl}_{3}\right)$ 26.4, 29.4, 34.7, 45.9, 49.7, 55.3, 112.7, $119.7,122.5,126.5,127.4,127.5,128.4,130.1,132.5,134.7,138.2,144.1,153.5 ; \mathrm{HRMS} \mathrm{C}_{19} \mathrm{H}_{20} \mathrm{~N}_{3}{ }^{35} \mathrm{Cl}_{2}[\mathrm{M}+\mathrm{H}]^{+}$calcd 360.1029 , obsd 360.1028 .

(S)-5-Chloro-2-(3-methoxybenzyl)-1-(piperidin-3-yl)-1H-benzo[d]imidazole 15\{3\}.

General Procedure B: $14\{3\}$ (0.514 g, 1.127 mmol). 15\{3\} (0.38 g, 95\%), white solid; ${ }^{1} \mathrm{H}$ NMR (500 MHz, $\left.\mathrm{CDCl}_{3}\right) 1.45$ $(1 \mathrm{H}, \mathrm{qt}, J 4.0$ and 13.0), $1.58(1 \mathrm{H}$, app.d), $1.64(1 \mathrm{H}, \mathrm{br} \mathrm{s}, \mathrm{NH}), 1.75-1.79(1 \mathrm{H}, \mathrm{m}), 2.13(1 \mathrm{H}, \mathrm{qt}, J 4.2$ and 12.8), $2.58(1 \mathrm{H}$, $\mathrm{td}, J 2.6$ and 12.3), $2.83(1 \mathrm{H}, \mathrm{dd}, J 3.7$ and 11.6), 3.02-3.05 $(1 \mathrm{H}, \mathrm{m}), 3.24(1 \mathrm{H}, \mathrm{t}, J 11.4), 3.74(3 \mathrm{H}, \mathrm{s}, \mathrm{OCH}), 4.22-4.28$ $(1 \mathrm{H}, \mathrm{m}), 4.28(1 \mathrm{H}, \mathrm{d}, J$ 15.9, -CHHAr), $4.34(1 \mathrm{H}, \mathrm{d}, J$ 15.8, -CHHAr), 6.76-6.78 (2H, m, Ar), 6.80-6.82 (1H, m, Ar), 7.16 $\left(1 \mathrm{H}, \mathrm{dd}, J 2.0\right.$ and 8.8, Ar), 7.19-7.23 (1H, m, Ar), $7.42(1 \mathrm{H}, \mathrm{d}, J$ 8.8, $\mathrm{Ar}), 7.72(1 \mathrm{H}, \mathrm{d}, J 2.0) ;{ }^{13} \mathrm{C} \mathrm{NMR}\left(125 \mathrm{MHz}, \mathrm{CDCl}_{3}\right)$ 
26.3, 29.2, 35.2, 45.8, 49.4, 55.0, 55.3, 112.6, 112.6, 114.0, 119.7, 120.7, 122.3, 127.3, 129.8, 132.5, 137.7, 144.2, 154.3, 160.0; $\mathrm{HRMS} \mathrm{C}{ }_{20} \mathrm{H}_{23} \mathrm{~N}_{3} \mathrm{O}^{35} \mathrm{Cl}[\mathrm{M}+\mathrm{H}]^{+}$calcd 356.1524, obsd 356.1526.

(S)-5-Chloro-2-(4-methoxybenzyl)-1-(piperidin-3-yl)-1H-benzo[d]imidazole 15\{4\}.

General Procedure B: $14\{4\}$ (0.376 g, 0.825 mmol). 15\{4\} (0.29 g, 99\%), white solid; ${ }^{1} \mathrm{H} \mathrm{NMR}(500 \mathrm{MHz}, \mathrm{CDCl} 3) 1.45$ $\left(1 \mathrm{H}, \mathrm{qt}, J 4.0\right.$ and $\left.12.9,15-\mathrm{H}_{\mathrm{ax}}\right), 1.58\left(1 \mathrm{H}, \mathrm{app} . \mathrm{d}, 14-\mathrm{H}_{\mathrm{eq}}\right), 1.76-1.80\left(2 \mathrm{H}, \mathrm{m}, 15-\mathrm{H}_{\mathrm{eq}}\right.$ and $\left.\mathrm{NH}\right), 2.13(1 \mathrm{H}, \mathrm{qd}, J 4.1$ and $\left.12.7,14-\mathrm{H}_{\mathrm{ax}}\right), 2.58\left(1 \mathrm{H}, \mathrm{td}, J 2.6\right.$ and $\left.12.4,16-\mathrm{H}_{\mathrm{ax}}\right), 2.80\left(1 \mathrm{H}, \mathrm{dd}, J 3.8\right.$ and 11.6, 12- $\left.\mathrm{H}_{\mathrm{eq}}\right), 3.02-3.06\left(1 \mathrm{H}, \mathrm{m}, 16-\mathrm{H}_{\mathrm{eq}}\right), 3.23$ $\left(1 \mathrm{H}, \mathrm{t}, J 11.5,12-\mathrm{H}_{\mathrm{ax}}\right), 3.77\left(3 \mathrm{H}, \mathrm{s}, \mathrm{OCH}_{3}\right), 4.21-4.27\left(1 \mathrm{H}, \mathrm{m}, 13-\mathrm{H}_{\mathrm{ax}}\right), 4.24(1 \mathrm{H}, \mathrm{d}, J 15.8,-\mathrm{CH} H \mathrm{Ar}), 4.29(1 \mathrm{H}, \mathrm{d}, J 15.8,-$ CHHAr), $6.82(2 \mathrm{H}, \mathrm{d}, J 8.8,2 \times 10-\mathrm{H}), 7.14(2 \mathrm{H}, \mathrm{d}, J$ 8.8, $2 \times 9-\mathrm{H}), 7.15(1 \mathrm{H}, \mathrm{dd}, J 2.0$ and 8.8, 5-H), 7.42 (1H, d, J 8.8, 6$\mathrm{H}) ; 7.72(1 \mathrm{H}, \mathrm{d}, \mathrm{J} 2.0,3-\mathrm{H}) ;{ }^{13} \mathrm{C}$ NMR (125 MHz, CDCl $) 26.6$ (C-15), 29.3 (C-14), $34.3\left(\mathrm{CH}_{2}\right), 46.0$ (C-16), 49.6 (C-12), $55.3\left(\mathrm{OCH}_{3}\right.$ and C-13), 112.6 (C-6), 114.2 (C-10), 119.6 (C-3), 122.2 (C-5), 127.2 (C-4), 128.2 (C-8), 129.4 (C-9), 132.5 (C-7), 144.2 (C-2), 154.8 (C-1), 158.6 (C-11); HRMS for $\mathrm{C}_{20} \mathrm{H}_{23} \mathrm{~N}_{3} \mathrm{O}^{35} \mathrm{Cl}[\mathrm{M}+\mathrm{H}]^{+}$calcd 356.1524, obsd 356.1521.

General procedure C. Either an aliquot of a solution of 15 in dry DCM ( $1 \mathrm{~mL}, 0.049 \mathrm{M})$, or weighed 15 in dry DCM (1 $\mathrm{mL}$ ) was treated with the named isocyanate (1.5 eq.). The mixture was quenched with $\mathrm{NH}_{2}$-silica and filtered through a plug of $\mathrm{NH}_{2}$-silica. Evaporation gave $\mathbf{8}$ without further purification.

(S)-3-(5-Chloro-2-benzyl-1H-benzo[d]imidazol-1-yl)-N-(4-nitrophenyl)piperidine-1-carboxamide 8\{1,1\}.

General procedure C: $15\{2\}$ (29 mg, $89 \mu \mathrm{mol})$ and 4-nitrophenyl isocyanate $(22 \mathrm{mg}, 134 \mu \mathrm{mol}) .8\{1,1\}(31 \mathrm{mg}, 71 \%)$, yellow solid; HPLC purity (as area \%) > 97; ${ }^{1} \mathrm{H}$ NMR (500 MHz, CDCl $) 1.43(1 \mathrm{H}, \mathrm{qt}, \mathrm{J} 4.0$ and 13.1), 1.58 (1H, app.d), $1.80(1 \mathrm{H}$, app. d), $2.14(1 \mathrm{H}, \mathrm{qd}, J 4.1$ and 12.8), $2.76(1 \mathrm{H}, \mathrm{td}, J 2.5$ and 13.3), $3.37(1 \mathrm{H}, \mathrm{t}, J 12.2), 3.81(1 \mathrm{H}, \mathrm{m}), 4.17-$ $4.28(4 \mathrm{H}, \mathrm{m}), 7.04(1 \mathrm{H}$, app.t, Ar), 7.10-7.20 (4H, m, Ar), $7.21(1 \mathrm{H}, \mathrm{s}, \mathrm{NH}), 7.29(1 \mathrm{H}, \mathrm{d}, \mathrm{J} 8.7, \mathrm{Ar}), 7.45(2 \mathrm{H}, \mathrm{d}, \mathrm{J} 9.2, \mathrm{Ar})$, $7.67(1 \mathrm{H}, \mathrm{d}, \mathrm{J} 2.0, \mathrm{Ar}), 8.07$ (2H, d, J 9.2, Ar); ${ }^{13} \mathrm{C}$ NMR (125 MHz, CDCl $)$ 24.9, 28.6, 35.2, 44.2, 47.2, 53.6, 112.2, 118.8, $119.9,122.8,124.9,127.4,127.9,128.4,129.0,132.2,135.9,142.6,144.1,145.5,153.9,154.4 ; M S(E S I+) \mathrm{m} / \mathrm{z}=$ 490.3. HRMS calcd for $\mathrm{C}_{26} \mathrm{H}_{24}{ }^{35} \mathrm{CIN}_{5} \mathrm{O}_{3}[\mathrm{M}+\mathrm{H}]^{+} 490.1640$, obsd 490.1627

(S)-3-(5-Chloro-2-benzyl-1H-benzo[d]imidazol-1-yl)-N-(4-acetylphenyl)piperidine-1-carboxamide 8\{1,2\}.

General procedure C: $15\{1\}(30 \mathrm{mg}, 92 \mu \mathrm{mol})$ and 4-acetylphenyl isocyanate $(22 \mathrm{mg}, 138 \mu \mathrm{mol}) .8\{1,2\}(27 \mathrm{mg}, 60 \%)$, white solid; HPLC purity (as area \%) > 96; ${ }^{1} \mathrm{H}$ NMR (500 MHz, CDCl $) 1.53(1 \mathrm{H}, \mathrm{qt}, J 4.0$ and 13.3), 1.72 (1H, app.d), 1.88-1.91 $(1 \mathrm{H}, \mathrm{m}), 2.26\left(1 \mathrm{H}, \mathrm{qd}, J 4.1\right.$ and 12.8), $2.57\left(3 \mathrm{H}, \mathrm{s}, \mathrm{COCH}_{3}\right), 2.85(1 \mathrm{H}, \mathrm{td}, J 2.6$ and 13.3$), 3.42(1 \mathrm{H}, \mathrm{app} . \mathrm{t})$, $3.68(1 \mathrm{H}, \mathrm{dd}, J 4.3$ and 12.6), $4.16(1 \mathrm{H}$, app.d), 4.28-4.34 $(2 \mathrm{H}, \mathrm{m}), 4.38(1 \mathrm{H}, \mathrm{d}, J 15.9), 6.25(1 \mathrm{H}, \mathrm{s}, \mathrm{NH}), 7.10(1 \mathrm{H}, \mathrm{tt}, J$ 1.5 and 7.0, Ar), $7.19(1 \mathrm{H}, \mathrm{dd}, \mathrm{J} 2.0$ and 8.7, $\mathrm{Ar}), 7.20-7.27(5 \mathrm{H}, \mathrm{m}, \mathrm{Ar}), 7.35-7.39(3 \mathrm{H}, \mathrm{m}, \mathrm{Ar}), 7.76(1 \mathrm{H}, \mathrm{d}, \mathrm{J} 2.0, \mathrm{Ar})$, $7.92(1 \mathrm{H}, \mathrm{d}, \mathrm{J}$ 8.8, $\mathrm{Ar}) ;{ }^{13} \mathrm{C} \mathrm{NMR}\left(125 \mathrm{MHz}, \mathrm{CDCl}_{3}\right)$ 24.9, 26.4, 28.6, 35.2, 44.3, 47.2, 53.5, 112.1, 118.7, 120.0, 122.8, $127.4,127.8,128.4,129.0,129.7,132.1,132.2,136.1,143.2,144.2,154.0,154.3,196.9 ; \mathrm{MS}(\mathrm{ESI}+) \mathrm{m} / \mathrm{z}=487.3$ $[\mathrm{M}+\mathrm{H}]^{+} ; \mathrm{HRMS} \mathrm{C}_{19} \mathrm{H}_{20} \mathrm{~N}_{3}{ }^{35} \mathrm{Cl}_{2}[\mathrm{M}+\mathrm{H}]^{+}$calcd 360.1029, obsd 360.1028.

(S)-3-(5-Chloro-2-benzyl-1H-benzo[d]imidazol-1-yl)-N-(3,4,5-trimethoxyphenyl)piperidine-1-carboxamide 8\{1,3\}.

General procedure C: $15\{1\}(27 \mathrm{mg}, 83 \mu \mathrm{mol})$ and 3,4,5-(trimethoxy)phenyl isocyanate $(26 \mathrm{mg}, 126 \mu \mathrm{mol}) .8\{1,3\}(16$ $\mathrm{mg}, 36 \%)$, white solid; HPLC purity (as area \%) > 94; ${ }^{1} \mathrm{H} \mathrm{NMR}\left(500 \mathrm{MHz}, \mathrm{CDCl}_{3}\right) 1.45(1 \mathrm{H}, \mathrm{qt}, \mathrm{J} 4.0$ and 13.2$), 1.61(1 \mathrm{H}$, app.d), 1.80 (1H, app.d), 2.15 (1H, qd, J 4.0 and 12.8), $2.75(1 \mathrm{H}, \mathrm{td}, J 2.3$ and 13.1), $3.32(1 \mathrm{H}, \mathrm{t}, \mathrm{J} 12.1), 3.62-3.65(1 \mathrm{H}$, m), $3.74\left(3 \mathrm{H}, \mathrm{s}, \mathrm{OCH}_{3}\right), 3.77\left(6 \mathrm{H}, \mathrm{s}, 2 \times \mathrm{OCH}_{3}\right), 4.06(1 \mathrm{H}, \mathrm{app} . \mathrm{d}), 4.21-4.26(1 \mathrm{H}, \mathrm{m}), 4.28\left(2 \mathrm{H}, \mathrm{s}, \mathrm{CH} \mathrm{Ph}^{\mathrm{P}}\right), 5.96(1 \mathrm{H}, \mathrm{s}$, $\mathrm{NH}), 6.52(1 \mathrm{H}, \mathrm{s}, \mathrm{Ar}), 7.08(1 \mathrm{H}, \mathrm{t}, \mathrm{J}$ 7.2, Ar), 7.12 (1H, dd, J 2.0 and 8.7, Ar), 7.15 (2H, app.d, Ar), 7.18-7.21 (2H, m, Ar), 
$7.29\left(1 \mathrm{H}, \mathrm{d}, \mathrm{J}\right.$ 8.7, Ar), $7.69(1 \mathrm{H}, \mathrm{d}, \mathrm{J} 2.0, \mathrm{Ar}) ;{ }^{13} \mathrm{C} \mathrm{NMR}\left(125 \mathrm{MHz}, \mathrm{CDCl}_{3}\right)$ 24.9, 28.6, 35.2, 44.2, 47.3, 53.6, 56.1, 61.0, 98.1, 112.2, 120.0, 122.7, 127.3, 127.8, 128.5, 129.0, 132.2, 134.3, 134.6, 136.1, 144.2, 153.3, 154.4, 154.9; MS (ESI+) $\mathrm{m} / \mathrm{z}=535.3[\mathrm{M}+\mathrm{H}]^{+} ; \mathrm{HRMS}$ for $\mathrm{C}_{29} \mathrm{H}_{32}{ }^{35} \mathrm{CIN}_{4} \mathrm{O}_{4}[\mathrm{M}+\mathrm{H}]^{+}$calcd 535.2107, obsd 535.2104.

(S)-3-(5-Chloro-2-benzyl-1H-benzo[d]imidazol-1-yl)-N-(4-trifluoromethoxyphenyl)piperidine-1-carboxamide 8\{1,4\}.

General procedure C: $15\{1\}(1 \mathrm{~mL}, 49 \mu \mathrm{mol})$ and 4-(trifluoromethoxy)phenyl isocyanate $(11 \mu \mathrm{l}, 78 \mu \mathrm{mol}) .8\{1,4\}(22$ $\mathrm{mg}, 82 \%)$, white solid; $\mathrm{HPLC}$ purity (as area \%) > 96; ${ }^{1} \mathrm{H} \mathrm{NMR}\left(500 \mathrm{MHz}, \mathrm{CDCl}_{3}\right) 1.50(1 \mathrm{H}, \mathrm{qt}, \mathrm{J} 4.1$ and 13.1), $1.67(1 \mathrm{H}$, app.d), 1.87 (1H, app.d), $2.22(1 \mathrm{H}, \mathrm{qd}, J 4.1$ and 12.8), 2.81 (1H, td, J 2.6 and 13.2), 3.39 (1H, app.t), 3.71 (1H, dd, J 4.4 and 12.7), $4.15(1 \mathrm{H}$, app.d), 4.25-4.34 (3H, m), $6.28(1 \mathrm{H}, \mathrm{s}, \mathrm{NH}), 7.10-7.20(6 \mathrm{H}, \mathrm{m}, \mathrm{Ar}), 7.23-7.26(2 \mathrm{H}, \mathrm{m}, \mathrm{Ar}), 7.30(2 \mathrm{H}$, d, J 9.1, Ar), $7.35\left(1 \mathrm{H}, \mathrm{d}, J\right.$ 8.7, Ar), $7.74(1 \mathrm{H}, \mathrm{d}, J 1.9, \mathrm{Ar}) ;{ }^{13} \mathrm{C} \mathrm{NMR}\left(125 \mathrm{MHz}, \mathrm{CDCl}_{3}\right)$ 24.8, 28.6, 35.1, 44.2, 47.2, 53.5, $112.2,119.9,120.5$ (q, J 256.6), 121.3, 121.6, 122.8, 127.3, 127.8, 128.4, 129.0, 132.2, 136.0, 137.3, 144.1, 144.8, 154.3, 154.6; $\left.{ }^{19} \mathrm{~F} \mathrm{NMR} \mathrm{(470.7} \mathrm{MHz,} \mathrm{CDCl}_{3}\right)-58.14 ; \mathrm{MS}(\mathrm{ESI}+) \mathrm{m} / \mathrm{z}=529.3[\mathrm{M}+\mathrm{H}]^{+}$.

(S)-3-(5-Chloro-2-benzyl-1H-benzo[d]imidazol-1-yl)-N-(4-trifluoromethylphenyl)piperidine-1-carboxamide 8\{1,5\}.

General procedure C: $15\{1\}$ (26 mg, $80 \mu \mathrm{mol}$ ) and 4-(trifluoromethyl)phenyl isocyanate (11.2 $\mu \mathrm{l}, 78.4 \mu \mathrm{mol}) .8\{1,5\}$ (20 mg, 79\%), white solid; HPLC purity (as area \%) > 97; $\left.{ }^{1} \mathrm{H} \mathrm{NMR} \mathrm{(500} \mathrm{MHz,} \mathrm{CDCl}\right) 1.52(1 \mathrm{H}, \mathrm{qt}, J 4.0$ and 13.2), 1.69 (1H, app.d), $1.88(1 \mathrm{H}$, app.d), $2.23(1 \mathrm{H}, \mathrm{qd}, J 4.1$ and 12.8), $2.83(1 \mathrm{H}, \mathrm{td}, J 2.5$ and 13.3), $3.40(1 \mathrm{H}, \mathrm{app} . \mathrm{t}), 3.71$ (1H, dd, J 4.3 and 12.6), $4.17(1 \mathrm{H}, \mathrm{d}, J$ 12.3), 4.26-4.33 (3H, m), $6.40(1 \mathrm{H}, \mathrm{s}, \mathrm{NH}), 7.11(1 \mathrm{H}$, app.t, Ar), 7.18-7.20 (3H, m, Ar), $7.24(2 \mathrm{H}$, app.t, $\mathrm{Ar}), 7.35(1 \mathrm{H}, \mathrm{d}, J$ 8.7, $\mathrm{Ar}), 7.41(2 \mathrm{H}, \mathrm{d}, J$ 8.6, $\mathrm{Ar}), 7.54(2 \mathrm{H}, \mathrm{d}, J$ 8.6, $\mathrm{Ar}), 7.75(1 \mathrm{H}, \mathrm{d}, J$ 2.0, $\mathrm{Ar}) ;{ }^{13} \mathrm{C}$ NMR (125 MHz, CDCl ) 24.9, 28.6, 35.2, 44.2, 47.2, 53.5, 112.2, 119.4, 119.9, 122.8, 126.2 (q, J 3.8), 127.4, 127.9, 128.4, 129.0, 132.2, 136.0, 141.9, 144.1, 154.3, 154.3; $\left.{ }^{19} \mathrm{~F} \mathrm{NMR} \mathrm{(470.7} \mathrm{MHz,} \mathrm{CDCl}\right)-61.9 ; \mathrm{MS}(\mathrm{ESI}+) \mathrm{m} / \mathrm{z}=513.3[\mathrm{M}+\mathrm{H}]^{+}$.

(S)-3-(5-Chloro-2-benzyl-1H-benzo[d]imidazol-1-yl)- $N$-phenylpiperidine-1-carboxamide 8 $\{1,6\}$.

General procedure C: $\mathbf{1 5}\{1\}(1 \mathrm{~mL}, 49 \mu \mathrm{mol})$ and phenyl isocyanate $(8 \mu \mathrm{l}, 76 \mu \mathrm{mol}) .8\{1,6\}(17 \mathrm{mg}, 77 \%)$ as a white solid; HPLC purity (as area \%) > 93; ${ }^{1} \mathrm{H}$ NMR $\left(500 \mathrm{MHz}, \mathrm{CDCl}_{3}\right) 1.50(1 \mathrm{H}, \mathrm{qt}, J 4.1$ and 13.1$), 1.66(1 \mathrm{H}$, app.d), 1.83-1.87 $(1 \mathrm{H}, \mathrm{m}), 2.23(1 \mathrm{H}, \mathrm{qd}, J 4.1$ and 12.8), $2.82(1 \mathrm{H}, \mathrm{td}, J 2.6$ and 13.3), $3.39(1 \mathrm{H}, \mathrm{app} . \mathrm{t}), 3.72(1 \mathrm{H}, \mathrm{dd}, J 4.5$ and 12.7), 4.11$4.14(1 \mathrm{H}, \mathrm{m}), 4.31(1 \mathrm{H}, \mathrm{dt}, J 4.5$ and 11.9), $4.34(2 \mathrm{H}, \mathrm{s}), 6.06(1 \mathrm{H}, \mathrm{s}, \mathrm{NH}), 7.07(1 \mathrm{H}, \mathrm{tt}, J 1.6$ and $7.0, \mathrm{Ar}), 7.12(1 \mathrm{H}, \mathrm{t}, J$ 7.2, $\mathrm{Ar}), 7.19(1 \mathrm{H}, \mathrm{dd}, J 2.1$ and 8.7, $\mathrm{Ar}), 7.21-7.32(8 \mathrm{H}, \mathrm{m}, \mathrm{Ar}), 7.37(1 \mathrm{H}, \mathrm{d}, J$ 8.7, $\mathrm{Ar}), 7.76(1 \mathrm{H}, \mathrm{d}, J 2.1, \mathrm{Ar}) ;{ }^{13} \mathrm{C} \mathrm{NMR}$ (125 MHz, $\left.\mathrm{CDCl}_{3}\right)$ 24.9, 28.6, 35.2, 44.3, 47.3, 53.5, 112.2, 119.9, 120.2, 122.7, 123.5, 127.3, 127.7, 128.4, 128.9, 129.0, 132.2, 136.1, 138.5, 144.2, 154.4, 154.9; MS (ESI+) m/z = 445.3 $[\mathrm{M}+\mathrm{H}]^{+} ; \mathrm{HRMS} \mathrm{C} \mathrm{C}_{26} \mathrm{H}_{26}{ }^{35} \mathrm{CIN} \mathrm{N}_{4} \mathrm{O}[\mathrm{M}+\mathrm{H}]^{+}$calcd 445.1790, obsd 445.1787.

(S)-Ethyl 3-(3-(5-chloro-2-benzyl-1H-benzo[d]imidazol-1-yl)piperidine-1-carboxamido)benzoate 8\{1,7\}.

General procedure C: $15\{1\}$ (22 mg, $67.7 \mu \mathrm{mol})$ and ethyl 3-isocyanatobenzoate (19 mg, $101 \mu \mathrm{mol}) .8\{1,7\}(17 \mathrm{mg}$, $49 \%$ ) as a white solid; HPLC purity (as area \%) > 99; ${ }^{1} \mathrm{H} \mathrm{NMR}\left(500 \mathrm{MHz}, \mathrm{CDCl}_{3}\right) 1.32\left(3 \mathrm{H}, \mathrm{t}, J 7.1, \mathrm{OCH}_{2} \mathrm{CH}_{3}\right), 1.46(1 \mathrm{H}$, qt, J 3.8 and 13.2), $1.63(1 \mathrm{H}$, app.d), $1.80(1 \mathrm{H}$, app.d), $2.18(1 \mathrm{H}, \mathrm{qd}, J 4.0$ and 12.8), $2.76(1 \mathrm{H}, \mathrm{td}, J 2.5$ and 13.2$), 3.34$ $(1 \mathrm{H}, \mathrm{t}, J$ 12.1), $3.64(1 \mathrm{H}, \mathrm{app} \mathrm{d}), 4.10(1 \mathrm{H}, \mathrm{app} \mathrm{d}), 4.23-4.35(5 \mathrm{H}, \mathrm{m}), 6.12(1 \mathrm{H}, \mathrm{s}, \mathrm{NH}), 7.08(1 \mathrm{H}, \mathrm{t}, J \mathrm{~J} .2, \mathrm{Ar}), 7.13(1 \mathrm{H}$, $\mathrm{dd}, J 2.0$ and 8.7, Ar), 7.15 (2H, app.d, Ar), 7.18-7.21 (2H, m, Ar), 7.28-7.31 (2H, m, Ar), 7.61-7.62 (1H, app dd, Ar), 7.66-7.71 (3H,m, Ar); $\left.{ }^{13} \mathrm{C} \mathrm{NMR} \mathrm{(125} \mathrm{MHz,} \mathrm{CDCl}\right)$ 14.4, 24.9, 28.6, 35.1, 44.2, 47.4, 53.6, 61.2, 112.3, 120.0, 120.9, $122.9,124.5,124.7,127.5,127.5,128.5,129.1,131.1,132.2,136.1,138.9,144.2,154.3,154.7,166.3 ; \mathrm{MS}$ (ESI+) m/z $=517.3[\mathrm{M}+\mathrm{H}]^{+}$.

(S)-Ethyl 4-(3-(5-chloro-2-benzyl-1H-benzo[d] imidazol-1-yl)piperidine-1-carboxamido)benzoate 8\{1,8\}. 
General procedure C: $15\{1\}(1 \mathrm{~mL}, 49.3 \mu \mathrm{mol})$ and ethyl 4-isothiocyanatobenzoate $(14.1 \mathrm{mg}, 75.6 \mu \mathrm{mol}) .8\{1,8\}$ (18 $\mathrm{mg}, 71 \%$ ) as a white solid; $\mathrm{HPLC}$ purity (as area \%) > 97; ${ }^{1} \mathrm{H} \mathrm{NMR}\left(500 \mathrm{MHz}, \mathrm{CDCl}_{3}\right) 1.38\left(3 \mathrm{H}, \mathrm{t}, \mathrm{J} 7.2, \mathrm{COOCH}_{2} \mathrm{CH}_{3}\right), 1.51$ $(1 \mathrm{H}, \mathrm{qt}, J 4.0$ and 13.3), 1.68 (1H, app.d), 1.88 (1H, app.d), 2.23 (1H, qd, J 4.0 and 12.9), $2.82(1 \mathrm{H}, \mathrm{td}, J 2.4$ and 13.3), $3.40\left(1 \mathrm{H}\right.$, app.t), $3.71(1 \mathrm{H}, \mathrm{dd}, J 4.1$ and 12.6$), 4.17\left(1 \mathrm{H}\right.$, app.d), 4.27-4.31 (3H, m), $4.35\left(2 \mathrm{H}, \mathrm{q}, J \mathrm{~J}, 2, \mathrm{COOCH}_{2} \mathrm{CH}_{3}\right)$, $6.42(1 \mathrm{H}, \mathrm{s}, \mathrm{NH}), 7.10(1 \mathrm{H}, \mathrm{t}, J$ J 7.3, Ar), 7.18-7.25 (5H, m, Ar), $7.34(1 \mathrm{H}, \mathrm{d}, J$ 8.7, Ar), $7.36(2 \mathrm{H}, \mathrm{d}, J$ 8.8, Ar), $7.75(1 \mathrm{H}, \mathrm{d}, J$ 2.0, $\mathrm{Ar}$ ), $7.97(2 \mathrm{H}, \mathrm{d}, \mathrm{J}$ 8.8, $\mathrm{Ar}) ;{ }^{13} \mathrm{C}$ NMR (125 MHz, CDCl 3 ) 14.4, 24.9, 28.6, 35.1, 44.2, 47.2, 53.5, 60.8, 112.2, 118.7, $119.9,122.8,125.0,127.4,127.8,128.4,129.0,130.7,132.2,136.0,143.0,144.1,154.1,154.3,166.2 ; \mathrm{MS}(\mathrm{ESI}+) \mathrm{m} / \mathrm{z}$ $=517.3[\mathrm{M}+\mathrm{H}]^{+} ; \mathrm{HRMS} \mathrm{C}_{29} \mathrm{H}_{30}{ }^{35} \mathrm{CIN}_{4} \mathrm{O}_{3}[\mathrm{M}+\mathrm{H}]^{+}$calcd 517.2001, obsd 517.2004.

(S)-3-(5-Chloro-2-benzyl-1H-benzo[d]imidazol-1-yl)-N-(4-fluorophenyl)piperidine-1-carboxamide 8\{1,9\}.

General procedure C: $15\{1\}$ ( $26 \mathrm{mg}, 80 \mu \mathrm{mol})$ and 4-fluorophenyl isocyanate (14 $\mu \mathrm{l}, 120 \mu \mathrm{mol}) .8\{1,9\}$ (22 mg, 60\%), white solid; HPLC purity (as area \%) > 90; ${ }^{1} \mathrm{H}$ NMR (500 MHz, CDCl 3 ) $1.43(1 \mathrm{H}, \mathrm{qt}, J 4.0$ and 13.2), 1.60 (1H, app.d), $1.78(1 \mathrm{H}, \mathrm{app} . \mathrm{d}), 2.15(1 \mathrm{H}, \mathrm{qd}, J 4.1$ and 12.9), $2.73(1 \mathrm{H}, \mathrm{td}, J 2.4$ and 13.1), $3.30(1 \mathrm{H}, \mathrm{t}, J 12.2), 3.61(1 \mathrm{H}, \mathrm{m}), 4.07(1 \mathrm{H}$, app.d), 4.19-4.24 (1H, m), $4.25(2 \mathrm{H}, \mathrm{s}), 6.03(1 \mathrm{H}, \mathrm{s}, \mathrm{NH}), 6.91(2 \mathrm{H}, \mathrm{app} . \mathrm{t}, \mathrm{Ar}), 7.04(1 \mathrm{H}, \mathrm{t}, J$ J 7.3 Ar), 7.11-7.19 (6H, m, $\mathrm{Ar}), 7.29(1 \mathrm{H}, \mathrm{d}, J$ 8.7, $\mathrm{Ar}), 7.68(1 \mathrm{H}, \mathrm{d}, J 2.0, \mathrm{Ar}) ;{ }^{13} \mathrm{C} \mathrm{NMR}\left(125 \mathrm{MHz}, \mathrm{CDCl}_{3}\right)$ 24.9, 28.6, 35.2, 44.2, 47.3, 53.5, 112.2, 115.5 (d, J $22.6 \mathrm{~Hz}$ ), 119.9, 122.4 (d, J $7.5 \mathrm{~Hz}$ ), 122.8, 127.3, 127.8, 128.4, 129.0, 132.2, 134.4, 136.1, 144.2, 154.3, 155.0, 159.2 (d, J 242.8 Hz); $\left.{ }^{19} \mathrm{~F} \mathrm{NMR} \mathrm{(376.2} \mathrm{MHz,} \mathrm{CDCl}\right)-119.3 ; \mathrm{MS}(\mathrm{ESI}+) \mathrm{m} / \mathrm{z}=463.3[\mathrm{M}+\mathrm{H}]^{+} ; \mathrm{HRMS} \mathrm{C}_{29} \mathrm{H}_{25}{ }^{35} \mathrm{ClFN}_{4} \mathrm{O}$ $[\mathrm{M}+\mathrm{H}]^{+}$calcd 463.1695, obsd 463.1695.

(S)-3-(5-Chloro-2-benzyl-1H-benzo[d]imidazol-1-yl)-N-cyclohexylpiperidine-1-carboxamide 8\{1,10\}.

General procedure C: $15\{1\}(1 \mathrm{~mL}, 49 \mu \mathrm{mol})$ and cyclohexyl isocyanate $(11 \mu \mathrm{l}, 76 \mu \mathrm{mol}) .8\{1,10\}(19 \mathrm{mg}, 85 \%)$, white solid; HPLC purity (as area \%) > 97; ${ }^{1} \mathrm{H}$ NMR $\left(500 \mathrm{MHz}, \mathrm{CDCl}_{3}\right) 1.00-1.08(2 \mathrm{H}, \mathrm{m}), 1.07(1 \mathrm{H}, \mathrm{qt}, J 3.6$ and 12.5), 1.31$1.45(3 \mathrm{H}, \mathrm{m}), 1.55$ (1H, app.d), 1.63 (1H, app.d), 1.69-1.72 (2H, m), 1.75-1.79 (1H, m), 1.88-1.95 (2H, m), $2.14(1 \mathrm{H}, \mathrm{qd}$, $J 12.8$ and 4.1), $2.72(1 \mathrm{H}, \mathrm{td}, J 13.1$ and 2.6), $3.28(1 \mathrm{H}, \mathrm{app} . \mathrm{t}), 3.56-3.63(1 \mathrm{H}, \mathrm{m}), 3.67(1 \mathrm{H}, \mathrm{dd}, J 12.6$ and 4.6$), 3.92$ $(1 \mathrm{H}, \mathrm{dd}, J 13.4$ and 1.5), $4.02(1 \mathrm{H}, \mathrm{d}, J$ J 7.5), $4.25(1 \mathrm{H}, \mathrm{dt}, J 12.0$ and 4.4), $4.32(1 \mathrm{H}, \mathrm{d}, J 15.9), 4.38(1 \mathrm{H}, \mathrm{d}, J$ 15.9), 7.17 $\left(1 \mathrm{H}, \mathrm{dd}, J 8.7\right.$ and 2.0, Ar), 7.21-7.32 (5H, m, Ar), $7.35(1 \mathrm{H}, \mathrm{d}, J$ 8.7, $\mathrm{Ar}), 7.75(1 \mathrm{H}, \mathrm{d}, J 2.0, \mathrm{Ar}) ;{ }^{13} \mathrm{C} \mathrm{NMR}(125 \mathrm{MHz}$, $\mathrm{CDCl}_{3}$ ) 24.7, 25.1, 25.6, 28.6, 33.9, 33.9, 35.2, 44.2, 47.1, 49.6, 53.5, 112.3, 119.9, 122.5, 127.3, 127.6, 128.4, 128.9, 132.3, 136.3, 144.2, 154.4, 156.9; MS (ESI+) m/z = $451.4[\mathrm{M}+\mathrm{H}]^{+}$.

\section{(S)-3-(5-Chloro-2-(3-chlorobenzyl)-1H-benzo[d] imidazol-1-yl)- $N$-(4-nitrophenyl)piperidine-1-carboxamide 8\{2,1\}.}

General procedure C: $15\{2\}(1 \mathrm{~mL}, 49.3 \mu \mathrm{mol})$ and 4-nitrophenyl isocyanate $(12.4 \mathrm{mg}, 75.6 \mu \mathrm{mol}) .8\{2,1\}(2 \mathrm{mg}, 8 \%)$, yellow solid; HPLC purity (as area \%) > 97; ${ }^{1} \mathrm{H} \mathrm{NMR}\left(500 \mathrm{MHz}, \mathrm{CDCl}_{3}\right) 1.49(1 \mathrm{H}, \mathrm{qt}, \mathrm{J} 4.0$ and 13.2), 1.66 (1H, app.d), $1.87(1 \mathrm{H}, \mathrm{app} . \mathrm{d}), 2.22$ (1H, qd, J 4.1 and 12.9), 2.88 (1H, td, J 2.3 and 13.3), 3.39 (1H, app.t), 3.88 (1H, app.d), 4.08 (1H, app.d), 4.17-4.25 (2H, m), 4.27 (1H, d, J 16.4), 6.73 (1H, br.s, NH), 7.02 (2H, app.t, Ar), 7.10-7.13 (2H, m, Ar), 7.16 $\left(1 \mathrm{H}, \mathrm{dd}, J 1.9\right.$ and 8.7, Ar), $7.32(1 \mathrm{H}, \mathrm{d}, J$ 8.7, $\mathrm{Ar}), 7.43(2 \mathrm{H}, \mathrm{d}, J$ 9.2, $\mathrm{Ar}), 7.67(1 \mathrm{H}, \mathrm{d}, J$ 1.9, $\mathrm{Ar}), 8.11\left(2 \mathrm{H}, \mathrm{d}, J\right.$ 9.2, Ar); ${ }^{13} \mathrm{C}$ NMR (125 MHz, CDCl$)$ 25.0, 28.6, 34.6, 44.6, 47.1, 53.4, 112.2, 118.7, 119.9, 123.1, 125.0, 126.5, 127.6, 128.1, 128.4, $130.3,132.0,134.9,137.8,142.8,143.8,144.9,153.4,153.6 ; \mathrm{MS}(\mathrm{ESI}+) \mathrm{m} / \mathrm{z}=524.2[\mathrm{M}+\mathrm{H}]^{+}$.

(S)-3-(5-Chloro-2-(3-chlorobenzyl)-1H-benzo[d]imidazol-1-yl)-N-(4-acetylphenyl)piperidine-1-carboxamide 8\{2,2\}.

General procedure C: $\mathbf{1 5}\{2\}(1 \mathrm{~mL}, 49.3 \mu \mathrm{mol})$ and 4-acetylphenyl isocyanate $(11.9 \mathrm{mg}, 75.6 \mu \mathrm{mol}) .8\{2,2\}(24 \mathrm{mg}$, 93\%), white solid; $\mathrm{HPLC}$ purity (as area \%) > 95; ${ }^{1} \mathrm{H} \mathrm{NMR}\left(400 \mathrm{MHz}, \mathrm{CDCl}_{3}\right) 1.51(1 \mathrm{H}, \mathrm{qt}, J 3.7$ and 12.8$), 1.65(1 \mathrm{H}$, app.dd), 1.87 (1H, app.d), $2.23\left(1 \mathrm{H}, \mathrm{qd}, J 4.1\right.$ and 12.8), $2.53\left(3 \mathrm{H}, \mathrm{s}, \mathrm{COCH}_{3}\right), 2.88(1 \mathrm{H}, \mathrm{td}, J 2.3$ and 13.7), 3.39 (1H, t, J 
11.9), $3.94(1 \mathrm{H}$, app. dd), $4.12(1 \mathrm{H}, \mathrm{app} . \mathrm{d}), 4.20-4.32(3 \mathrm{H}, \mathrm{m}), 6.80(1 \mathrm{H}, \mathrm{s}, \mathrm{NH}), 7.03-7.08(2 \mathrm{H}, \mathrm{m}, \mathrm{Ar}), 7.13(1 \mathrm{H}, \mathrm{d}, J$ 7.8, Ar), 7.16-7.20 (2H, m, Ar), $7.35(1 \mathrm{H}, \mathrm{d}, J$ 8.7, $\mathrm{Ar}), 7.40(2 \mathrm{H}, \mathrm{d}, J$ 8.7, $\mathrm{Ar}), 7.71(1 \mathrm{H}, \mathrm{d}, J$ 1.8, $\mathrm{Ar}), 7.87(2 \mathrm{H}, \mathrm{d}, J$ 8.7); ${ }^{13} \mathrm{C}$ NMR $\left(100 \mathrm{MHz}, \mathrm{CDCl}_{3}\right)$ 25.0, 26.5, 28.8, 34.7, 44.7, 47.2, 53.6, 118.8, 120.0,123.1, 126.6, 127.7, 127.9, 128.5, $129.8,130.3,132.1,134.9,137.9,143.5,153.5,154.3,197.1 ; \mathrm{MS}(\mathrm{ESI}+) \mathrm{m} / \mathrm{z}=521.3[\mathrm{M}+\mathrm{H}]^{+}$.

(S)-3-(5-Chloro-2-(3-chlorobenzyl)-1H-benzo[d]imidazol-1-yl)-N-(3,4,5-trimethoxyphenyl)piperidine-1-carboxamide $8\{2,3\}$.

General procedure C: $\mathbf{1 5}\{2\}(1 \mathrm{~mL}, 49.3 \mu \mathrm{mol})$ and 3,4,5-(trimethoxy)phenyl isocyanate $(16 \mathrm{mg}, 76 \mu \mathrm{mol}) .8\{2,3\}(27$ $\mathrm{mg}, 97 \%)$, white solid; $\mathrm{HPLC}$ purity (as area \%) > 97; ${ }^{1} \mathrm{H} \mathrm{NMR}\left(500 \mathrm{MHz}, \mathrm{CDCl}_{3}\right) 1.47(1 \mathrm{H}, \mathrm{qt}, \mathrm{J} 4.0$ and 13.2), $1.60(1 \mathrm{H}$, app.dd), $1.82(1 \mathrm{H}$, app.d), $2.18(1 \mathrm{H}, \mathrm{qd}, J 4.0$ and 12.8), $2.81(1 \mathrm{H}, \mathrm{td}, J 2.4$ and 13.2), $3.34(1 \mathrm{H}$, app.t), $3.73(3 \mathrm{H}, \mathrm{s}$, $\left.\mathrm{OCH}_{3}\right), 3.76\left(6 \mathrm{H}, \mathrm{s}, \mathrm{OCH}_{3}\right), 3.82(1 \mathrm{H}, \mathrm{app} . \mathrm{dd}), 4.03(1 \mathrm{H}, \mathrm{app} . \mathrm{d}), 4.16-4.23(2 \mathrm{H}, \mathrm{m}), 4.27(1 \mathrm{H}, \mathrm{d}, J$ 16.0), $6.21(1 \mathrm{H}, \mathrm{s}, \mathrm{NH})$, $6.54(2 \mathrm{H}, \mathrm{s}, \mathrm{Ar}), 7.02(1 \mathrm{H}, \mathrm{d}, J$ 7.8, Ar), 7.04-7.06 (1H, m, Ar), $7.10(1 \mathrm{H}, \mathrm{d}, J$ 7.8, Ar), 7.12-7.14 (2H, m, Ar), $7.31(1 \mathrm{H}, \mathrm{d}, J$ 8.7, $\mathrm{Ar}), 7.68(1 \mathrm{H}, \mathrm{d}, J 2.0, \mathrm{Ar}) ;{ }^{13} \mathrm{C}$ NMR $\left(125 \mathrm{MHz}_{\mathrm{CDCl}}\right.$ ) 25.0, 28.8, 34.7, 44.6, 47.2, 53.6, 56.1, 61.0, 98.1, 112.3, $120.0,122.9,126.6,127.6,127.9,128.5,130.2,132.2,134.3,134.7,134.8,138.0,144.1,153.3,153.5,154.9$; MS $(E S I+) m / z=569.3[M+H]^{+}$.

(S)-3-(5-Chloro-2-(3-chlorobenzyl)-1H-benzo[d] imidazol-1-yl)-N-(4-trifluoromethoxy)phenylpiperidine-1carboxamide $8\{2,4\}$.

General procedure C: $\mathbf{1 5}\{2\}(1 \mathrm{~mL}, 49 \mu \mathrm{mol})$ and 4-(trifluoromethoxy)phenyl isocyanate $(11 \mu \mathrm{l}, 76 \mu \mathrm{mol}) .8\{2,4\}(26$ $\mathrm{mg}, 94 \%)$, white solid; $\mathrm{HPLC}$ purity (as area \%) > 96; ${ }^{1} \mathrm{H} \mathrm{NMR}\left(500 \mathrm{MHz}, \mathrm{CDCl}_{3}\right) 1.53(1 \mathrm{H}, \mathrm{qt}, \mathrm{J} 4.0$ and 13.2), $1.69(1 \mathrm{H}$, app.d), 1.89 (1H, app.d), 2.22-2.29 (1H, m), 2.89 (1H, td, J 2.5 and 13.2), 3.40 (1H, app.t), 3.90-3.93 (1H, m), 4.09 (1H, app.d), 4.21-4.28 (1H, m), $4.25(1 \mathrm{H}, \mathrm{d}, J 16.1), 4.32(1 \mathrm{H}, \mathrm{d}, J 16.1), 6.44(1 \mathrm{H}$, br.s, NH), 7.06 (1H, app.d, Ar), 7.10 (1H, app.d, Ar), 7.14-7.19 (4H, m, Ar), $7.21(1 \mathrm{H}, \mathrm{dd}, J 2.0$ and 8.7, Ar), $7.32(2 \mathrm{H}, \mathrm{d}, J$ 9.1, Ar), $7.37(1 \mathrm{H}, \mathrm{d}, J$ 8.7, $\mathrm{Ar}), 7.74$ $(1 \mathrm{H}, \mathrm{d}, J$ 2 2.0, $\mathrm{Ar}) ;{ }^{13} \mathrm{C}$ NMR $\left(125 \mathrm{MHz}, \mathrm{CDCl}_{3}\right)$ 25.0, 28.7, 34.6, 44.6, 47.2, 53.5, 112.2, 120.5 (q, J 256.8), 120.0, 121.2, 121.7, 123.0, 126.5, 127.6, 127.9, 128.4, 130.2, 132.2, 134.8, 137.3, 137.9, 144.1, 144.8, 153.4, 154.7; ${ }^{19}$ F NMR (470.7 $\left.\mathrm{MHz}, \mathrm{CDCl}_{3}\right)-58.14 ; \mathrm{MS}(\mathrm{ESI}+) \mathrm{m} / \mathrm{z}=563.2[\mathrm{M}+\mathrm{H}]^{+}$.

(S)-3-(5-Chloro-2-(3-chlorobenzyl)-1H-benzo[d]imidazol-1-yl)- N-(4-trifluoromethylphenyl)piperidine-1carboxamide $8\{2,5\}$.

General procedure C: $15\{2\}(1 \mathrm{~mL}, 49 \mu \mathrm{mol})$ and 4-(trifluoromethyl)phenyl isocyanate $(11 \mu \mathrm{l}, 78 \mu \mathrm{mol}) .8\{2,5\}(23$ $\mathrm{mg}, 85 \%)$, white solid; $\mathrm{HPLC}$ purity (as area \%) > 96; ${ }^{1} \mathrm{H} \mathrm{NMR}\left(400 \mathrm{MHz}, \mathrm{CDCl}_{3}\right) 1.53(1 \mathrm{H}, \mathrm{qt}, J 4.1$ and 13.3$), 1.68(1 \mathrm{H}$, app.d), 1.89 (1H, app.d), 2.25 (1H, qd, J 4.1 and 12.7), 2.90 (1H, td, J 2.3 and 13.3), 3.42 (1H, app.t), 3.94 (1H, app.d), $4.10(1 \mathrm{H}, \mathrm{app} . \mathrm{d}), 4.23-4.31(2 \mathrm{H}, \mathrm{m}), 4.34(1 \mathrm{H}, \mathrm{d}, J 15.6), 6.49(1 \mathrm{H}, \mathrm{s}, \mathrm{NH}), 7.06-7.09(2 \mathrm{H}, \mathrm{m}, \mathrm{Ar}), 7.16(1 \mathrm{H}, \mathrm{app} . \mathrm{t}, \mathrm{Ar})$, 7.18-7.21 (2H, m, Ar), $7.31\left(1 \mathrm{H}, \mathrm{d}, J\right.$ 8.7, Ar), $7.42(2 \mathrm{H}, \mathrm{d}, J$ 8.7, $\mathrm{Ar}), 7.53\left(2 \mathrm{H}, \mathrm{d}, J\right.$ 8.7, Ar), $7.71(1 \mathrm{H}, \mathrm{s}, \operatorname{Ar}) ;{ }^{13} \mathrm{C}$ NMR $\left(75 \mathrm{MHz}_{1} \mathrm{CDCl}_{3}\right)$ 25.1, 28.9, 34.8, 45.0, 47.6, 53.7, 112.2, 119.5, 120.2, 125.4, 126.5 (q, J 3.8), 126.6, 127.7, 128.3, 128.6, 130.3, 132.3, 135.1, 138.2, 142.0, 153.5, 154.4; $\left.{ }^{19} \mathrm{~F} \mathrm{NMR} \mathrm{(376.2} \mathrm{MHz,} \mathrm{CDCl}\right)-61.9 ; \mathrm{MS}(\mathrm{ESI}+) \mathrm{m} / \mathrm{z}=547.3$.

(S)-3-(5-Chloro-2-(3-chlorobenzyl)-1H-benzo[d] imidazol-1-yl)-N-phenylpiperidine-1-carboxamide 8\{2,6\}.

General procedure C: $15\{2\}(1 \mathrm{~mL}, 49 \mu \mathrm{mol})$ and phenyl isocyanate $(8 \mu \mathrm{l}, 76 \mu \mathrm{mol}) .8\{2,6\}(23 \mathrm{mg}$, 98\%), white solid; HPLC purity (as area \%) > 98; ${ }^{1} \mathrm{H}$ NMR $\left(500 \mathrm{MHz}^{\mathrm{CDCl}}{ }_{3}\right) 1.43(1 \mathrm{H}, \mathrm{qt}, \mathrm{J} 4.0$ and 13.2), 1.56 (1H, app.dd), $1.78(1 \mathrm{H}$, app.d), $2.16(1 \mathrm{H}, \mathrm{qd}, J 4.0$ and 12.8), $2.79(1 \mathrm{H}, \mathrm{td}, J 2.5$ and 13.2), $3.30(1 \mathrm{H}, \mathrm{app} . \mathrm{t}), 3.87(1 \mathrm{H}, \mathrm{app} . \mathrm{dd}), 4.01(1 \mathrm{H}$, app.d), 4.15-4.20 (2H, m), $4.25(1 \mathrm{H}, \mathrm{d}, J$ 16.1), $6.37(1 \mathrm{H}, \mathrm{s}, \mathrm{NH}), 6.98-7.04(3 \mathrm{H}, \mathrm{m}, \mathrm{Ar}), 7.07-7.12(2 \mathrm{H}, \mathrm{m}, \mathrm{Ar}), 7.13(1 \mathrm{H}$, $\mathrm{dd}, J 2.0$ and 7.8, Ar), 7.22-7.23 (4H, m, Ar), $7.30(1 \mathrm{H}, \mathrm{d}, J$ 8.7, $\mathrm{Ar}), 7.67(1 \mathrm{H}, \mathrm{d}, J 2.0, \mathrm{Ar}) ;{ }^{13} \mathrm{C} \mathrm{NMR}\left(125 \mathrm{MHz}, \mathrm{CDCl}_{3}\right)$ 
25.0, 28.8, 34.6, 44.7, 47.3, 53.5, 112.3, 120.0, 120.3, 122.9, 123.6, 126.5, 127.6, 127.9, 128.5, 129.0, 130.2, 132.2, $134.9,138.0,138.6,144.1,153.6,155.1 ; \mathrm{MS}(\mathrm{ESI}+) \mathrm{m} / \mathrm{z}=479.3[\mathrm{M}+\mathrm{H}]^{+}$.

(S)-Ethyl 3-(3-(5-chloro-2-(3-chlorobenzyl)-1H-benzo[d]imidazol-1-yl)piperidine-1-carboxamido)benzoate 8\{2,7\}. General procedure C: $15\{2\}(1 \mathrm{~mL}, 49 \mu \mathrm{mol})$ and 3-isothiocyanatobenzoate $(12 \mu \mathrm{l}, 76 \mu \mathrm{mol}) .8\{2,7\}(22 \mathrm{mg}, 81 \%)$, white solid; HPLC purity (as area \%) > 98; ${ }^{1} \mathrm{H}$ NMR $\left(500 \mathrm{MHz}, \mathrm{CDCl}_{3}\right) 1.37\left(3 \mathrm{H}, \mathrm{t}, \mathrm{J} 7.1, \mathrm{CH}_{2} \mathrm{CH}_{3}\right), 1.53(1 \mathrm{H}, \mathrm{qt}, \mathrm{J} 4.0$ and 13.3), 1.66 (1H, app.d), 1.87 (1H, app.d), 2.25 (1H, qd, J 4.1 and 12.8), 2.88 (1H, td, J 2.4 and 13.3), 3.41 (1H, app.t), $3.95(1 \mathrm{H}$, app.d), 4.13 (1H, app.d), 4.24-4.30 (1H, m), $4.28(1 \mathrm{H}, \mathrm{d}, J 16.1), 4.34(1 \mathrm{H}, \mathrm{d}, J 16.1), 4.34(2 \mathrm{H}, \mathrm{d}, J 7.1$, $\left.\mathrm{CH}_{2} \mathrm{CH}_{3}\right), 6.60(1 \mathrm{H}, \mathrm{s}, \mathrm{NH})$, 7.07-7.11 (2H, m, Ar), 7.15-7.19 (2H, m, Ar), 7.20 (1H, d, J 8.7, Ar), 7.34-7.39 (2H, m, Ar), $7.68\left(1 \mathrm{H}, \mathrm{ddd}, \mathrm{J}\right.$ 1.0, 2.3 and 8.7, Ar), 7.71-7.74 (2H, m, Ar), 7.80 (1H, app.t, Ar); $\left.{ }^{13} \mathrm{C} \mathrm{NMR} \mathrm{(125} \mathrm{MHz,} \mathrm{CDCl}\right)$ 14.3, 24.9, 28.7, 34.6, 44.6, 47.2, 53.5, 61.1, 112.3, 119.9, 120.9, 123.0, 124.5, 124.6, 126.5, 127.6, 127.9, 128.4, 129.0, 130.2, 131.1, 132.1, 134.8, 137.9, 138.9, 153.5, 154.8, 166.3; MS (ESI+) m/z = 551.3 [M+H] $]^{+}$

(S)-Ethyl 4-(3-(5-chloro-2-(3-chlorobenzyl)-1H-benzo[d]imidazol-1-yl)piperidine-1-carboxamido)benzoate 8\{2,8\}. General procedure C: $15\{2\}(1 \mathrm{~mL}, 49 \mu \mathrm{mol})$ and 4-isocyanatobenzoate (14 mg, $76 \mu \mathrm{mol}) .8\{2,8\}(26 \mathrm{mg}, 96 \%)$, white solid; HPLC purity (as area \%) > 96; ${ }^{1} \mathrm{H}$ NMR (400MHz, $\left.\mathrm{CDCl}_{3}\right) 1.35\left(3 \mathrm{H}, \mathrm{t}, \mathrm{J}\right.$ 7.3, $\left.\mathrm{CH}_{2} \mathrm{CH}_{3}\right), 1.49(1 \mathrm{H}, \mathrm{qt}, \mathrm{J} 4.1$ and 13.3$)$, 1.65 (1H, app.d), 1.85 (1H, app.d), 2.21 (1H, qd, J 4.1 and 12.8), 2.84 (1H, td, J 2.3 and 13.3), 3.37 (1H, app.t), 3.91 (1H, app. dd), $4.11\left(1 \mathrm{H}\right.$, app.d), $4.19(1 \mathrm{H}, \mathrm{d}, J 16.0), 4.27(1 \mathrm{H}, \mathrm{d}, J 16.0), 4.31\left(2 \mathrm{H}, \mathrm{q}, J 7.3, \mathrm{CH}_{2} \mathrm{CH}_{3}\right), 6.80(1 \mathrm{H}, \mathrm{s}, \mathrm{NH})$, 7.02-7.07 (2H, m, Ar), 7.10-7.14 (2H, m, Ar), $7.17(1 \mathrm{H}, \mathrm{d}, J 1.8$ and 8.7, Ar), $7.34(1 \mathrm{H}, \mathrm{d}, J$ 8.7, Ar), 7.37 (2H, d, J 8.7, Ar), $7.71(1 \mathrm{H}, \mathrm{d}, J 1.8, \mathrm{Ar}), 7.93(2 \mathrm{H}, \mathrm{d}, J$ 8.7, $\mathrm{Ar}) ;{ }^{13} \mathrm{C} \mathrm{NMR}\left(100 \mathrm{MHz}, \mathrm{CDCl}_{3}\right)$ 14.3, 24.9, 28.7, 34.5, 44.6, 47.1, 53.5, $60.8,112.2$, 118.7, 119.9, 123.0, 124.9, 126.5, 127.6, 127.9, 128.4, 130.2, 130.6, 132.1, 134.8, 137.8, 143.1, 143.9, 153.4, 154.3, 166.3; MS (ESI+) $\mathrm{m} / \mathrm{z}=551.3[\mathrm{M}+\mathrm{H}]^{+}$.

(S)-3-(5-Chloro-2-(3-chlorobenzyl)-1H-benzo[d] imidazol-1-yl)-N-(4-fluorophenyl)piperidine-1-carboxamide 8\{2,9\}.

General procedure C: $15\{2\}(1 \mathrm{~mL}, 49 \mu \mathrm{mol})$ and 4-fluorophenyl isocyanate $(8 \mu \mathrm{l}, 76 \mu \mathrm{mol}) .8\{2,9\}(16 \mathrm{mg}, 65 \%)$, white solid; HPLC purity (as area \%) > 97; ${ }^{1} \mathrm{H}$ NMR (500 MHz, CDCl $) 1.52(1 \mathrm{H}, \mathrm{qt}, J 4.1$ and 13.2$), 1.67$ (1H, app.d), $1.87(1 \mathrm{H}$, app.d), 2.25 (1H, qd, J 4.3 and 12.7), 2.87 (1H, td, J 2.6 and 13.3), 3.39 (1H, app.t), 3.88-3.92 (1H, m), 4.08 (1H, app.d), 4.22-4.26 (1H, m), 4.26 (1H,d, J 16.0), 4.32 (1H,d, J 16.0), 6.32 (1H, br.s, NH), 6.99 (2H, app.t, Ar), 7.07 (1H, app.d, Ar), 7.10-7.12 (1H, m, Ar), 7.15-7.25 (5H, m, Ar), $7.38\left(1 \mathrm{H}, \mathrm{d}, J\right.$ 8.7, Ar), $7.74(1 \mathrm{H}, \mathrm{d}, \mathrm{J} 2.0, \mathrm{Ar}) ;{ }^{13} \mathrm{C} \mathrm{NMR}$ $\left(125 \mathrm{MHz}_{1} \mathrm{CDCl}_{3}\right)$ 25.0, 28.7, 34.5, 44.6, 47.2, 53.5, 112.3, 115.5 (d, J 22.5), 120.0, 122.3 (d, J 7.9), 122.9, 126.5, 127.6, $127.9,128.4,130.2,132.2,134.4$ (d, J 2.8), 134.8, 138.0, 144.1, 153.4, 155.0, 159.2 (d, J 242.8); ${ }^{19} \mathrm{~F} \mathrm{NMR} \mathrm{(470.7} \mathrm{MHz,}$ $\left.\mathrm{CDCl}_{3}\right)$-119.23; $\mathrm{MS}(\mathrm{ESI}+) \mathrm{m} / \mathrm{z}=497.2$.

\section{(S)-3-(5-Chloro-2-(3-chlorobenzyl)-1H-benzo[d]imidazol-1-yl)-N-cyclohexylpiperidine-1-carboxamide 8\{2,10\}.}

General procedure C: $15\{2\}(1 \mathrm{~mL}, 49 \mu \mathrm{mol})$ and cyclohexyl isocyanate (9 $\mu \mathrm{l}, 76 \mu \mathrm{mol}) . \mathbf{8}\{2,10\}(25 \mathrm{mg}, 99 \%)$, white solid; HPLC purity (as area \%) > 96; ${ }^{1} \mathrm{H}$ NMR (500 MHz, CDCl $)$ 0.95-1.04 (2H, m), 1.07 (1H, qt, J 12.5 and 3.5), $1.25-$ $1.34(2 \mathrm{H}, \mathrm{m}), 1.36$ (1H, qt, J 13.1 and 4.0), 1.47 (1H, app.d), 1.54-1.58 (1H, m), 1.62-1.66 (2H, m), 1.71-1.73 (1H, m), $1.87(2 \mathrm{H}, \mathrm{dd}, J 12.4$ and 2.5), $2.10(1 \mathrm{H}, \mathrm{qd}, J 12.8$ and 4.1), $2.73(1 \mathrm{H}, \mathrm{td}, J 13.2$ and 2.5), 3.22 (1H, t, J 12.1), 3.52-3.60 $(1 \mathrm{H}, \mathrm{m}), 3.78(1 \mathrm{H}$, app.d), $3.85(1 \mathrm{H}, \mathrm{dd}, J 12.6$ and 4.3), $4.14(1 \mathrm{H}, \mathrm{tt}, J 12.0$ and 4.3), 4.18 (1H, app.d), 4.21 (1H, d, J 16.0), $4.32(1 \mathrm{H}, \mathrm{d}, J$ 16.0), 7.02-7.05 (1H, m, Ar), 7.11 (1H, dd, J 8.7 and 2.0, Ar), 7.14-7.18 (3H, m, Ar), 7.31 (1H, d, J 8.7, $\mathrm{Ar}), 7.67(1 \mathrm{H}, \mathrm{d}, J$ 2.0, $\mathrm{Ar}) ;{ }^{13} \mathrm{C} \mathrm{NMR}\left(125 \mathrm{MHz}, \mathrm{CDCl}_{3}\right)$ 24.8, 25.0, 25.6, 28.7, 33.9, 33.9, 34.6, 44.6, 47.1, 49.6, $53.4,112.3,119.8,122.8,126.5,127.5,127.7,128.5,130.1,132.2,134.7,138.1,144.0,153.5,156.9 ; \mathrm{MS}(\mathrm{ESI}+) \mathrm{m} / \mathrm{z}=$ $485.3[\mathrm{M}+\mathrm{H}]^{+}$. 
(S)-3-(5-Chloro-2-(3-methoxybenzyl)-1H-benzo[d]imidazol-1-yl)-N-(4-nitrophenyl)piperidine-1-carboxamide 8\{3,1\}.

General procedure C: $\mathbf{1 5}\{3\}(1 \mathrm{~mL}, 49.3 \mu \mathrm{mol})$ and 4-nitrophenyl isocyanate (12 mg, $76 \mu \mathrm{mol}) .8\{3,1\}(19 \mathrm{mg}, 74 \%)$, white solid; HPLC purity (as area \%) > 95; ${ }^{1} \mathrm{H}$ NMR (500 MHz,CDCl$) 1.48$ (1H, qt, $J 3.9$ and 13.2), 1.68 (1H, app.dd), $1.86\left(1 \mathrm{H}\right.$, app.d), $2.17(1 \mathrm{H}, \mathrm{qd}, J 4.0$ and 12.8$), 2.79(1 \mathrm{H}, \mathrm{td}, J 2.4$ and 13.2$), 3.37(1 \mathrm{H}, \mathrm{t}, \mathrm{app} . \mathrm{t}), 3.59\left(3 \mathrm{H}, \mathrm{s}, \mathrm{OCH}_{3}\right), 3.72$ $\left(1 \mathrm{H}\right.$, app. dd), $4.17\left(2 \mathrm{H}\right.$, br.s, $\left.\mathrm{CH}_{2}\right), 4.25(1 \mathrm{H}, \mathrm{tt}, J 4.4$ and 12.0), $6.58(1 \mathrm{H}, \mathrm{dd}, J 2.1$ and 8.2, $\mathrm{Ar}), 6.64-6.67(2 \mathrm{H}, \mathrm{m}, \mathrm{Ar})$, $6.83(1 \mathrm{H}$, br.s, NH), $7.08(1 \mathrm{H}$, app.t, Ar), $7.12(1 \mathrm{H}, \mathrm{dd}, J 2.0$ and 8.7, $\mathrm{Ar}), 7.27(1 \mathrm{H}, \mathrm{d}, J$ 8.7, Ar), $7.43(2 \mathrm{H}, \mathrm{d}, J$ 9.2, $\mathrm{Ar})$, $7.65\left(1 \mathrm{H}, \mathrm{d}, J\right.$ 2.0, Ar), 8.08 (2H, d, J 9.2, Ar); ${ }^{13} \mathrm{C}$ NMR (125 MHz, CDCl $)$ 24.9, 28.6, 35.1, 44.2, 47.2, 53.5, 55.3, 60.4, $112.2,112.6,114.4,118.8,119.8,120.6,122.9,125.0,128.0,130.0,132.1,137.4,142.8,144.0,145.1$, 153.7, 154.1, 160.1 .

(S)-N-(4-Acetylphenyl)-3-(5-chloro-2-(3-methoxybenzyl)-1H-benzo[d]imidazol-1-yl)piperidine-1-carboxamide $8\{3,2\}$.

General procedure C: $15\{3\}(1 \mathrm{~mL}, 49.3 \mu \mathrm{mol})$ and 4-acetylphenyl isocyanate $(12 \mathrm{mg}, 76 \mu \mathrm{mol}) .8\{3,2\}(21 \mathrm{mg}, 82 \%)$, white solid; HPLC purity (as area \%) > 96; ${ }^{1} \mathrm{H}$ NMR $\left(400 \mathrm{MHz}, \mathrm{CDCl}_{3}\right) 1.50(1 \mathrm{H}, \mathrm{qt}, J 3.7$ and 12.8), $1.68(1 \mathrm{H}$, app.dd), $1.86\left(1 \mathrm{H}\right.$, app.d); 2.20(1H, qd, J 3.7 and 13.3), $2.53\left(3 \mathrm{H}, \mathrm{s}, \mathrm{COCH}_{3}\right), 2.80(1 \mathrm{H}, \mathrm{td}, J 2.3$ and 13.3$), 3.38(1 \mathrm{H}, \mathrm{t}, J$ 11.9), $3.61\left(3 \mathrm{H}, \mathrm{s}, \mathrm{OCH}_{3}\right), 3.77\left(1 \mathrm{H}\right.$, app. dd), $4.19(1 \mathrm{H}, \mathrm{app} . \mathrm{d}), 4.25\left(2 \mathrm{H}, \mathrm{s}, \mathrm{CH}_{2}\right), 4.30(1 \mathrm{H}, \mathrm{tt}, J 4.1$ and 11.9$), 6.62(1 \mathrm{H}$, $\mathrm{dd}, J 2.3$ and 8.2, Ar), 6.69-6.73 (3H, m, Ar + NH), 7.12(1H, app.t, Ar), $7.16(1 \mathrm{H}, \mathrm{dd}, J 1.8$ and 8.7, Ar), $7.32(1 \mathrm{H}, \mathrm{d}, J$ 8.7, $\mathrm{Ar}), 7.39(2 \mathrm{H}, \mathrm{d}, J$ 8.2, $\mathrm{Ar}), 7.70(1 \mathrm{H}, \mathrm{d}, J 1.8, \mathrm{Ar}), 7.86(2 \mathrm{H}, \mathrm{d}, J 8.2) ;{ }^{13} \mathrm{C} \mathrm{NMR}\left(100 \mathrm{MHz}, \mathrm{CDCl}_{3}\right) 25.0,26.5,28.7$, $35.1,44.3,47.3,53.6,55.3,112.3,112.7,114.4,118.9,119.9,120.6,122.9,127.9,129.7,130.0,132.0,132.2,137.5$, $143.5,143.9,154.2,160.1,197.1$.

(S)-3-(5-Chloro-2-(3-methoxybenzyl)-1H-benzo[d]imidazol-1-yl)-N-(3,4,5-trimethoxyphenyl)piperidine-1carboxamide $8\{3,3\}$.

General procedure C: $15\{3\}(1 \mathrm{~mL}, 49.3 \mu \mathrm{mol})$ and 3,4,5-trimethoxyphenyl isocyanate (15 mg, $76 \mu \mathrm{mol}) .8\{3,3\}(24$ $\mathrm{mg}, 87 \%)$, white solid; $\mathrm{HPLC}$ purity (as area \%) > 98; ${ }^{1} \mathrm{H} \mathrm{NMR}\left(500 \mathrm{MHz}, \mathrm{CDCl}_{3}\right) 1.46(1 \mathrm{H}, \mathrm{qt}, \mathrm{J} 4.0$ and 13.2), $1.65(1 \mathrm{H}$, app.dd), $1.80(1 \mathrm{H}$, app.d), $2.16(1 \mathrm{H}, \mathrm{qd}, J 4.0$ and 12.8), $2.73(1 \mathrm{H}, \mathrm{td}, J 2.4$ and 13.2), $3.32(1 \mathrm{H}, \mathrm{t}, \mathrm{app} . \mathrm{t}), 3.57$ (3H, s, $\left.\mathrm{OCH}_{3}\right), 3.63(1 \mathrm{H}, \mathrm{app} . \mathrm{dd}), 3.73\left(3 \mathrm{H}, \mathrm{s}, \mathrm{OCH}_{3}\right), 3.76(6 \mathrm{H}, \mathrm{s}, \mathrm{OCH}), 4.10(1 \mathrm{H}, \mathrm{app} . \mathrm{d}), 4.21(1 \mathrm{H}, \mathrm{d}, J$ J 16.2), 4.23-4.28 $(2 \mathrm{H}$, m), $6.10(1 \mathrm{H}, \mathrm{s}, \mathrm{NH}), 6.53(2 \mathrm{H}, \mathrm{s}, \mathrm{Ar}), 6.59(1 \mathrm{H}, \mathrm{dd}, J 2.0$ and 8.3, $\mathrm{Ar}), 6.68-6.71(2 \mathrm{H}, \mathrm{m}, \mathrm{Ar}), 7.08(1 \mathrm{H}, \mathrm{d}, J \mathrm{~J}, 9, \mathrm{Ar}), 7.11$ $(1 \mathrm{H}, \mathrm{dd}, J 2.0$ and 8.7, $\mathrm{Ar}), 7.29(1 \mathrm{H}, \mathrm{d}, J$ 8.7, $\left.\mathrm{Ar}), 7.67(1 \mathrm{H}, \mathrm{d}, J 2.0, \mathrm{Ar}) ;{ }^{13} \mathrm{C} \mathrm{NMR} 125 \mathrm{MHz}, \mathrm{CDCl}_{3}\right)$ 24.9, 28.7, 35.2, $44.2,47.3,53.6,55.2,56.1,61.0,98.3,112.2,112.7,114.2,119.9,120.6,122.7,127.7,130.0,132.2,134.3,134.7$, $137.6,144.1,153.3,154.2,155.0,160.0$.

(S)-3-(5-Chloro-2-(3-methoxybenzyl)-1H-benzo[d]imidazol-1-yl)-N-(4-trifluoromethoxyphenyl) piperidine-1carboxamide $8\{3,4\}$.

General procedure C: $\mathbf{1 5}\{3\}(1 \mathrm{~mL}, 49.3 \mu \mathrm{mol})$ and 4-(trifluoromethoxy)phenyl isocyanate $(11 \mathrm{mg}, 75 \mu \mathrm{mol}) . \mathbf{8}\{3,4\}$ (27 mg, 98\%) as a white solid; HPLC purity (as area \%) > 98; ${ }^{1} \mathrm{H} \mathrm{NMR}\left(500 \mathrm{MHz}, \mathrm{CDCl}_{3}\right) 1.49(1 \mathrm{H}, \mathrm{qt}, \mathrm{J} 4.0$ and 13.2), 1.68 (1H, app.dd), 1.84 (1H, app.d), 2.19 (1H, qd, J 4.1 and 12.8), 2.77 (1H, td, J 2.4 and 13.3), 3.36 (1H, app.t), 3.60 $\left(3 \mathrm{H}, \mathrm{s}, \mathrm{OCH}_{3}\right), 3.75$ (1H, app. dd), 4.16 (1H, app.d), 4.19-4.35 (3H, m), 6.53 (1H, br.d), 6.60-6.62 (1H, m, Ar), 6.69-6.70 $(2 \mathrm{H}, \mathrm{m}, \mathrm{Ar}), 7.09-7.14(3 \mathrm{H}, \mathrm{m}, \mathrm{Ar}), 7.16(1 \mathrm{H}, \mathrm{dd}, J 2.0$ and 8.7, Ar), $7.29(2 \mathrm{H}, \mathrm{d}, J$ 9.1, Ar), $7.32(1 \mathrm{H}, \mathrm{d}, J$ 8.7, Ar), 7.69 $(1 \mathrm{H}, \mathrm{d}, J$ 2.0, $\mathrm{Ar}) ;{ }^{13} \mathrm{C} \mathrm{NMR}\left(125 \mathrm{MHz}, \mathrm{CDCl}_{3}\right)$ 24.8; 28.6; 35.0; 44.1; 47.2; 53.2; 55.1; 112.2; 112.5; $114.3 ; 119.7$; 
120.5(q, J 256.6); 120.5; 121.4; 121.6; 122.8; 127.8; 129.9; 132.1; 137.4; 137.4; 143.8; 144.7; 154.1; 154.7; ${ }^{19} \mathrm{~F}$ NMR (470.7 MHz, $\mathrm{CDCl}_{3}$ ) -58.1.

(S)-3-(5-Chloro-2-(3-methoxybenzyl)-1H-benzo[d]imidazol-1-yl)-N-(4-trifluoromethylphenyl)piperidine-1carboxamide $8\{3,5\}$

General procedure C: $\mathbf{1 5}\{3\}(1 \mathrm{~mL}, 49.3 \mu \mathrm{mol})$ and 4-(trifluoromethyl)phenyl isocyanate $(11.2 \mu \mathrm{l}, 78.4 \mu \mathrm{mol}) .8\{3,5\}$ (23 mg, 85\%) as a white solid; HPLC purity (as area \%) > 96; ${ }^{1} \mathrm{H} \mathrm{NMR}\left(400 \mathrm{MHz}, \mathrm{CDCl}_{3}\right) 1.53(1 \mathrm{H}, \mathrm{qt}, \mathrm{J} 4.1$ and 13.1$)$, $1.74(1 \mathrm{H}, \mathrm{app} . \mathrm{d}), 1.89$ (1H, app.d), 2.24 (1H, qd, J 4.1 and 12.8), $2.82(1 \mathrm{H}, \mathrm{td}, J 2.3$ and 13.1), 3.40 (1H, app.t), 3.61 $\left(3 \mathrm{H}, \mathrm{s}, \mathrm{OCH}_{3}\right), 3.71$ (1H, app.d), 4.17 (1H, app.d), $4.26(1 \mathrm{H}, \mathrm{d}, J 16.0), 4.29(1 \mathrm{H}, \mathrm{d}, J$ 16.0), 4.28-4.35 (1H, m), $6.33(1 \mathrm{H}$, s, NH), $6.62(1 \mathrm{H}, \mathrm{dd}, J 2.3$ and 8.7, $\mathrm{Ar}), 6.73-6.75(2 \mathrm{H}, \mathrm{m}, \mathrm{Ar}), 7.14(1 \mathrm{H}, \mathrm{app} . \mathrm{t}, \mathrm{Ar}), 7.18(1 \mathrm{H}, \mathrm{dd}, J 1.8$ and 8.7), 7.35 $(1 \mathrm{H}, \mathrm{d}, J$ 8.7, $\mathrm{Ar}), 7.39(2 \mathrm{H}, \mathrm{d}, J$ 8.7, $\mathrm{Ar}), 7.52(2 \mathrm{H}, \mathrm{d}, J$ 8.7, $\mathrm{Ar}), 7.72(1 \mathrm{H}, \mathrm{d}, J 1.8, \mathrm{Ar}) ;{ }^{13} \mathrm{C} \mathrm{NMR}\left(75 \mathrm{MHz}, \mathrm{CDCl}_{3}\right) 25.0$, 28.8, 35.3, 44.6, 47.6, 53.7, 55.3, 112.1, 112.9, 114.5, 119.5, 120.2, 120.7, 122.9, 126.1 (q, J 3.8), 128.1, 130.0, 132.4, 132.4, 137.8, 142.1, 144.4, 154.2, 154.3, 160.4; $\left.{ }^{19} \mathrm{~F} \mathrm{NMR} \mathrm{(376.2} \mathrm{MHz,} \mathrm{CDCl}_{3}\right)$-61.9.

\section{(S)-3-(5-Chloro-2-(3-methoxybenzyl)-1H-benzo[d]imidazol-1-yl)-N-phenylpiperidine-1-carboxamide 8\{3,6\}}

General procedure C: $\mathbf{1 5}\{3\}(1 \mathrm{~mL}, 49.3 \mu \mathrm{mol})$ and phenyl isocyanate $(8.1 \mu \mathrm{l}, 75.6 \mu \mathrm{mol}) .8\{3,6\}(23 \mathrm{mg}, 98 \%)$ as a white solid; HPLC purity (as area \%) > 97; ${ }^{1} \mathrm{H}$ NMR $\left(500 \mathrm{MHz}, \mathrm{CDCl}_{3}\right) 1.55(1 \mathrm{H}, \mathrm{qt}, \mathrm{J} 4.0$ and 13.2), 1.74 (1H, app.dd), $1.89(1 \mathrm{H}, \mathrm{m}), 2.27\left(1 \mathrm{H}, \mathrm{qd}, J 4.0\right.$ and 12.8), $2.84\left(1 \mathrm{H}, \mathrm{td}, J 2.6\right.$ and 13.2), $3.41(1 \mathrm{H}, \mathrm{t}, \mathrm{app} . \mathrm{t}), 3.65\left(3 \mathrm{H}, \mathrm{s}, \mathrm{OCH}_{3}\right), 3.76$ $(1 \mathrm{H}, \mathrm{dd}, J 4.4$ and 12.7), $4.18(1 \mathrm{H}, \mathrm{br} . \mathrm{d}), 4.32(1 \mathrm{H}, \mathrm{d}, J$ 16.0), 4.34-4.39( 2H, m), $6.16(1 \mathrm{H}, \mathrm{br} . \mathrm{s}, \mathrm{NH}), 6.67(1 \mathrm{H}, \mathrm{dd}, J 2.0$ and 8.2, Ar), 6.78-6.80 (2H, m, Ar), $7.09(1 \mathrm{H}, \mathrm{tt}, J 2.1$ and 6.5, Ar), $7.19(1 \mathrm{H}$, app.t, Ar), 7.12(1H, dd, J 2.0 and 8.6, Ar), $7.29(4 \mathrm{H}, \mathrm{m}, \mathrm{Ar}), 7.39(1 \mathrm{H}, \mathrm{d}, J$ 8.6, $\mathrm{Ar}), 7.78(1 \mathrm{H}, \mathrm{d}, J$ 2.0, $\mathrm{Ar}) ;{ }^{13} \mathrm{C} \mathrm{NMR}\left(125 \mathrm{MHz}, \mathrm{CDCl}_{3}\right)$ 24.9, 28.7, 35.2, 44.3, 47.3, 53.5, 55.2, 112.2, 112.6, 114.3, 119.9, 120.3, 120.6, 122.7, 123.5, 127.7, 128.9, 129.9, 132.2, 137.6, 138.6, 144.2, 154.2, 154.9, 160.0 .

(S)-Ethyl 3-(3-(5-chloro-2-(3-methoxybenzyl)-1H-benzo[d]imidazol-1-yl)piperidine-1-carboxamido)benzoate 8\{3,7\}.

General procedure C: $15\{3\}(1 \mathrm{~mL}, 49.3 \mu \mathrm{mol})$ and ethyl 3-isothiocyanatobenzoate $(12 \mu \mathrm{l}, 76 \mu \mathrm{mol}) .8\{3,7\}(26 \mathrm{mg}$, 96\%) as a white solid; $\mathrm{HPLC}$ purity (as area \%) $>98 ;{ }^{1} \mathrm{H} \mathrm{NMR}\left(300 \mathrm{MHz}, \mathrm{CDCl}_{3}\right) 1.38\left(1 \mathrm{H}, \mathrm{t}, J \mathrm{f.2}, \mathrm{CH}_{2} \mathrm{CH}_{3}\right), 1.54(1 \mathrm{H}, \mathrm{qt}, J$ 3.6 and 13.1), $1.72(1 \mathrm{H}$, app.dd), $1.87(1 \mathrm{H}$, app.d), $2.20(1 \mathrm{H}, \mathrm{qd}, J 4.0$ and 12.7$), 2.80(1 \mathrm{H}, \mathrm{td}, J 2.2$ and 13.1$), 3.38(1 \mathrm{H}$, app.t), $3.63\left(3 \mathrm{H}, \mathrm{s}, \mathrm{OCH}_{3}\right), 3.77\left(1 \mathrm{H}\right.$, app. dd), $4.21(1 \mathrm{H}, \mathrm{app} . \mathrm{d}), 4.30\left(2 \mathrm{H}, \mathrm{s}, \mathrm{CH}_{2}\right), 4.36(2 \mathrm{H}, \mathrm{q}, J \mathrm{~J} .2), 6.54(1 \mathrm{H}, \mathrm{br} . \mathrm{d})$, 6.65-6.69 (1H, m, Ar), $6.77(2 \mathrm{H}, \mathrm{d}, J$ 8.3, Ar), $7.17(1 \mathrm{H}, \mathrm{app.t}, \mathrm{Ar}), 7.20(1 \mathrm{H}, \mathrm{m}, \mathrm{Ar}), 7.32-7.39(2 \mathrm{H}, \mathrm{m}, \mathrm{Ar}), 7.67-7.81$ $(4 \mathrm{H}, \mathrm{m}, \mathrm{Ar}) ;{ }^{13} \mathrm{C}$ NMR $\left(100 \mathrm{MHz}, \mathrm{CDCl}_{3}\right)$ 14.3, 24.8, 28.6, 35.0, 44.1, 47.2, 53.5, 55.2, 61.1, 112.2, 112.5, 114.3, 119.8, $120.5,121.0,122.7,124.4,124.7,127.7,128.9,129.9,131.0,132.1,137.5,138.9,144.0,154.2,154.7,160.0,166.3$.

(S)-Ethyl 4-(3-(5-chloro-2-(3-methoxybenzyl)-1H-benzo[d]imidazol-1-yl)piperidine-1-carboxamido)benzoate 5\{3,8\}.

General procedure C: $15\{3\}(1 \mathrm{~mL}, 49.3 \mu \mathrm{mol})$ and ethyl 4-isothiocyanatobenzoate $(14.1 \mathrm{mg}, 75.6 \mu \mathrm{mol}) .8\{3,8\}(23$ $\mathrm{mg}, 85 \%)$, white solid; $\mathrm{HPLC}$ purity (as area \%) > 97; ${ }^{1} \mathrm{H} \mathrm{NMR}\left(400 \mathrm{MHz}, \mathrm{CDCl}_{3}\right) 1.34\left(3 \mathrm{H}, \mathrm{t}, \mathrm{J} 7.6, \mathrm{CH}_{2} \mathrm{CH}_{3}\right), 1.45-1.55$ $(1 \mathrm{H}, \mathrm{m}), 1.69$ (1H, app.d), 1.85 (1H, app.d), 2.14-2.25 (1H, m), 2.79 (1H, app.t), 3.37 (1H, app.t), 3.60 (3H, s, OCH $)$, 3.71-3.75 $(1 \mathrm{H}, \mathrm{m}), 4.17(1 \mathrm{H}, \mathrm{app} . \mathrm{d}), 4.24-4.34(5 \mathrm{H}, \mathrm{m}), 6.58(1 \mathrm{H}, \mathrm{s}, \mathrm{NH}), 6.58(1 \mathrm{H}, \mathrm{dd}, J 2.3$ and 8.2, Ar), $6.72(2 \mathrm{H}$, app.d, Ar), $7.11(1 \mathrm{H}$, app.t, $\mathrm{Ar}), 7.16(1 \mathrm{H}, \mathrm{dd}, J 2.4$ and 8.7, $\mathrm{Ar}), 7.32(1 \mathrm{H}, \mathrm{d}, J$ 8.7, Ar), $7.36(1 \mathrm{H}, \mathrm{d}, J$ 8.7, $\mathrm{Ar}), 7.70(1 \mathrm{H}$, app.t, $\mathrm{Ar}), 7.93(2 \mathrm{H}, \mathrm{dd}, J 1.4$ and 8.7, $\mathrm{Ar}) ;{ }^{13} \mathrm{C} \mathrm{NMR}\left(100 \mathrm{MHz}, \mathrm{CDCl}_{3}\right)$ 14.3, 24.9, 28.6, 35.1, 44.2, 47.2, 53.5, 55.2, $60.8,112.2,112.5,114.3,118.7,119.8,120.5,122.8,127.8,130.0,130.6,132.1,137.4,143.1,144.0,154.1,154.2$, $160.0,166.2$. 
(S)-3-(5-Chloro-2-(3-methoxybenzyl)-1H-benzo[d]imidazol-1-yl)-N-(4-fluorophenyl)piperidine-1-carboxamide $8\{3,9\}$.

General procedure C: $15\{3\}(1 \mathrm{~mL}, 49.3 \mu \mathrm{mol})$ and 4-fluorophenyl isocyanate (8.4 $\mu \mathrm{l}, 75.6 \mu \mathrm{mol}) .8\{3,9\}(23 \mathrm{mg}, 96 \%)$, white solid; HPLC purity (as area \%) > 97; ${ }^{1} \mathrm{H}$ NMR (400 MHz, $\left.\mathrm{CDCl}_{3}\right) 1.50(1 \mathrm{H}, \mathrm{qt}, J 4.1$ and 13.3), 1.69 (1H, app.d), $1.83(1 \mathrm{H}, \mathrm{app} . \mathrm{d}), 2.20(1 \mathrm{H}, \mathrm{qd}, J 4.1$ and 12.6$), 2.76(1 \mathrm{H}, \mathrm{td}, J 2.3$ and 13.3$), 3.35(1 \mathrm{H}, \mathrm{app} . \mathrm{t}), 3.61\left(3 \mathrm{H}, \mathrm{s}, \mathrm{OCH}_{3}\right), 3.67-$ $3.72(1 \mathrm{H}, \mathrm{m}), 4.17(1 \mathrm{H}$, app.d), 4.12-4.16 (1H, app.d), 4.21-4.32 (2H, m), $6.26(1 \mathrm{H}, \mathrm{s}, \mathrm{NH}), 6.61(1 \mathrm{H}, \mathrm{dd}, J 2.3$ and 8.7, Ar), 6.71-6.73 (2H, app.d, Ar), $6.95(2 \mathrm{H}$, app.t, $\mathrm{Ar}), 7.10-7.21(4 \mathrm{H}, \mathrm{m}, \mathrm{Ar}), 7.33(1 \mathrm{H}, \mathrm{d}, J$ 8.7, $\mathrm{Ar}), 7.71(1 \mathrm{H}, \mathrm{d}, J$ 8.7, $\mathrm{Ar})$;

${ }^{13} \mathrm{C}$ NMR $\left(100 \mathrm{MHz}, \mathrm{CDCl}_{3}\right)$ 24.9, 28.6, 35.0, 44.1, 47.3, 53.5, 55.2, 112.2, 112.4, 114.3, 115.4 (d, J $\left.22.0 \mathrm{~Hz}\right), 119.8$, 120.5, 122.5 (d, J 7.7 Hz), 122.7, 127.7, 129.9, 132.2, 134.5, 134.5(d, J 2.9), 137.5, 144.0, 154.1, 155.0, 159.1 (d, J $243.5 \mathrm{~Hz}), 160.0 ;{ }^{19} \mathrm{~F} \mathrm{NMR}\left(376.2 \mathrm{MHz}, \mathrm{CDCl}_{3}\right)$-119.2.

(S)-3-(5-Chloro-2-(3-methoxybenzyl)-1H-benzo[d] imidazol-1-yl)-N-cyclohexylpiperidine-1-carboxamide 8\{3,10\}.

General procedure C: 15\{3\} (1 mL, $49 \mu \mathrm{mol})$ and cyclohexyl isocyanate $(9.4 \mu \mathrm{l}, 76 \mu \mathrm{mol}) .8\{3,10\}(24 \mathrm{mg}, 99 \%)$, white solid; HPLC purity (as area \%) > 93; ${ }^{1} \mathrm{H}$ NMR $\left(500 \mathrm{MHz}, \mathrm{CDCl}_{3}\right)$ 0.94-1.02 $(2 \mathrm{H}, \mathrm{m}), 1.06(1 \mathrm{H}, \mathrm{qt}, J 12.5$ and 3.5), 1.23$1.40(3 \mathrm{H}, \mathrm{m}), 1.50(1 \mathrm{H}$, app.d), $1.55(1 \mathrm{H}, \mathrm{dt}, J 12.9$ and 3.5), 1.62-1.65 $(2 \mathrm{H}, \mathrm{m}), 1.71(1 \mathrm{H}, \mathrm{app} \mathrm{dt}), 1.81-1.88(2 \mathrm{H}, \mathrm{m})$, $2.07(1 \mathrm{H}, \mathrm{qd}, J 12.8$ and 4.1), $2.67(1 \mathrm{H}, \mathrm{td}, J 13.2$ and 2.6), $3.22(1 \mathrm{H}, \mathrm{t}, J 12.1), 3.53(1 \mathrm{H}, \mathrm{dtt}, J$ 14.7, 10.9 and 3.9), 3.67 (3H, s, OCH $), 3.70$ (1H, dd, J 12.5 and 4.3), 3.85 (1H, app.d), 4.09 (1H, app.d), 4.18-4.21 (1H, m), 4.21 (1H, d, J 15.8), $4.30(1 \mathrm{H}, \mathrm{d}, J$ 15.8), 6.70-6.71 (3H, m, Ar), 7.10 (1H, dd, J 8.7 and 2.0, Ar), 7.14 (1H, app.t, Ar), 7.29 (1H, d, J 8.7, Ar), $7.67(1 \mathrm{H}, \mathrm{d}, J \mathrm{~J} .0, \mathrm{Ar}) ;{ }^{13} \mathrm{C}$ NMR $\left(125 \mathrm{MHz}, \mathrm{CDCl}_{3}\right)$ 24.7, 25.0, 25.1, 25.6, 28.6, 33.8, 33.9, 35.0, 44.2, 47.1, 49.6, 53.4, $55.2,112.3,112.9,113.9,119.8,120.6,122.6,127.6,129.9,132.2,137.6,144.0,154.2,156.9,160.0$.

(S)-3-(5-Chloro-2-(4-methoxybenzyl)-1H-benzo[d] imidazol-1-yl)-N-(4-acetylphenyl)piperidine-1-carboxamide $8\{4,2\}$.

General procedure C: $15\{4\}(1 \mathrm{~mL}, 49 \mu \mathrm{mol})$ and 4-acetylphenyl isocyanate $(12 \mathrm{mg}, 76 \mu \mathrm{mol}) . \mathbf{8}\{4,2\}(13 \mathrm{mg}, 51 \%)$ as a white solid; HPLC purity (as area \%) > 98; $\left.{ }^{1} \mathrm{H} \mathrm{NMR} \mathrm{(400MHz,} \mathrm{CDCl}\right) 1.51(1 \mathrm{H}, \mathrm{qt}, J 4.1$ and 13.3), 1.67 (1H, app.d), $1.87(1 \mathrm{H}, \mathrm{app} . \mathrm{d}), 2.20\left(1 \mathrm{H}, \mathrm{qd}, J 4.1\right.$ and 12.8), $2.53\left(3 \mathrm{H}, \mathrm{s}, \mathrm{COCH}_{3}\right), 2.82(1 \mathrm{H}, \mathrm{td}, J 2.3$ and 13.3), 3.39 (1H, app.t), 3.59 $\left(3 \mathrm{H}, \mathrm{s}, \mathrm{OCH}_{3}\right), 3.79(1 \mathrm{H}, \mathrm{dd}, J 4.1$ and 12.4), $4.18(1 \mathrm{H}, \mathrm{d}, J 16.0), 4.23(1 \mathrm{H}, \mathrm{d}, J 16.0), 4.30(1 \mathrm{H}, \mathrm{tt}, J 4.6$ and 11.9), 6.69 $(1 \mathrm{H}, \mathrm{s}, \mathrm{NH}), 6.75(2 \mathrm{H}, \mathrm{d}, J$ 8.7, Ar), $7.16(1 \mathrm{H}, \mathrm{dd}, J 2.3$ and 8.7, $\mathrm{Ar}), 7.32(2 \mathrm{H}, \mathrm{d}, J$ 8.7, Ar), $7.39(1 \mathrm{H}, \mathrm{d}, J$ 8.7, $\mathrm{Ar}), 7.70$ $\left(1 \mathrm{H}, \mathrm{d}, J\right.$ 2.3, Ar), $7.85(1 \mathrm{H}, \mathrm{d}, J$ 8.7, $\mathrm{Ar}) ;{ }^{13} \mathrm{C}$ NMR $\left(100 \mathrm{MHz}, \mathrm{CDCl}_{3}\right)$ 24.9, 26.4, 28.6, 34.2, 44.2, 47.2, 53.5, 55.1, 112.1, $114.3,118.7,119.8,122.7,127.7,127.8,129.4,129.6,131.2,143.4,143.9,154.2,154.6,158.7,197.0$.

(S)-3-(5-Chloro-2-(4-methoxybenzyl)-1H-benzo[d]imidazol-1-yl)-N-(3,4,5-trimethoxyphenyl)piperidine-1carboxamide $8\{4,3\}$.

General procedure C: $\mathbf{1 5}\{4\}(1 \mathrm{~mL}, 49 \mu \mathrm{mol})$ and 3,4,5-(trimethoxy)phenyl isocyanate (16 mg, $76 \mu \mathrm{mol}) .8\{4,3\}(26$ $\mathrm{mg}, 93 \%)$, white solid; HPLC purity (as area \%) > 95; ${ }^{1} \mathrm{H} \mathrm{NMR}\left(400 \mathrm{MHz}, \mathrm{CDCl}_{3}\right) 1.53(1 \mathrm{H}, \mathrm{qt}, \mathrm{J} 4.1$ and 13.3$), 1.70(1 \mathrm{H}$, app.d), $1.87(1 \mathrm{H}$, app.d), $2.22(1 \mathrm{H}, \mathrm{qd}, J 4.1$ and 12.8), $2.79(1 \mathrm{H}, \mathrm{td}, J 2.3$ and 13.3), $3.38(1 \mathrm{H}, \mathrm{t}, J 11.9), 3.54(3 \mathrm{H}, \mathrm{s}$, $\left.\mathrm{OCH}_{3}\right), 3.68(1 \mathrm{H}, \mathrm{m}), 3.78\left(3 \mathrm{H}, \mathrm{s}, \mathrm{OCH}_{3}\right), 3.81\left(6 \mathrm{H}, \mathrm{s}, 2 \times \mathrm{OCH}_{3}\right), 4.17(1 \mathrm{H}, \mathrm{app} . \mathrm{d}), 4.24(1 \mathrm{H}, \mathrm{d}, J 16.5), 4.28(1 \mathrm{H}, \mathrm{d}, J$ 16.5), $4.32(1 \mathrm{H}, \mathrm{tt}, J 4.1$ and 11.9), $6.09(1 \mathrm{H}, \mathrm{s}, \mathrm{NH}), 6.58(2 \mathrm{H}, \mathrm{s}, \mathrm{Ar}), 6.75(2 \mathrm{H}, \mathrm{d}, J$ 8.7, Ar), $7.11(2 \mathrm{H}, \mathrm{d}, J$ 8.7, $\mathrm{Ar}), 7.16$ $(1 \mathrm{H}, \mathrm{dd}, J 1.8$ and 8.7, $\mathrm{Ar}), 7.35(2 \mathrm{H}, \mathrm{d}, J$ 8.7, $\mathrm{Ar}), 7.71(1 \mathrm{H}, \mathrm{d}, J 1.8, \mathrm{Ar}) ;{ }^{13} \mathrm{C} \mathrm{NMR}\left(100 \mathrm{MHz}, \mathrm{CDCl}_{3}\right)$ 25.0, 28.7, 34.3, $44.1,47.4,53.7,55.1,56.1,61.0,98.1,112.3,114.4,119.9,122.8,127.8,129.5,132.2,134.2,134.6,144.2,153.3$, $154.7,155.0,158.8$. 
(S)-3-(5-Chloro-2-(4-methoxybenzyl)-1H-benzo[d]imidazol-1-yl)-N-(4-trifluoromethoxyphenyl)piperidine-1carboxamide $8\{4,4\}$.

General procedure C: $15\{4\}(1 \mathrm{~mL}, 49 \mu \mathrm{mol})$ and 4-(trifluoromethoxy)phenyl isocyanate $(11 \mu \mathrm{l}, 76 \mu \mathrm{mol}) .8\{4,4\}(27$ $\mathrm{mg}, 98 \%$ ) as a white solid; $\mathrm{HPLC}$ purity (as area \%) > 96; ${ }^{1} \mathrm{H} \mathrm{NMR}\left(500 \mathrm{MHz}, \mathrm{CDCl}_{3}\right) 1.54(1 \mathrm{H}, \mathrm{qt}, J 4.0$ and 13.1), 1.74 (1H, app.d), 1.89 (1H, app.d), 2.20-2.28 (1H, m), $2.83\left(1 \mathrm{H}, \mathrm{td}, \mathrm{J} 2.2\right.$ and 13.2), 3.39 (1H, app.t), $3.57\left(3 \mathrm{H}, \mathrm{s}, \mathrm{OCH}_{3}\right), 3.67$ $(1 \mathrm{H}, \mathrm{dd}, J 4.0$ and 12.5), 4.20 (1H, app.d), 4.20-4.23 (2H, m), $4.30(1 \mathrm{H}, \mathrm{tt}, J 4.3$ and 12.0), $6.28(1 \mathrm{H}, \mathrm{s}, \mathrm{NH}), 6.76(2 \mathrm{H}, \mathrm{d}$, J 8.7, $\mathrm{Ar}), 7.09(2 \mathrm{H}, \mathrm{d}, J$ 8.6, $\mathrm{Ar}), 7.13(2 \mathrm{H}, \mathrm{d}, J$ 8.6, $\mathrm{Ar}), 7.18(1 \mathrm{H}, \mathrm{d}, J 1.9$ and 8.7, $\mathrm{Ar}), 7.30(2 \mathrm{H}, \mathrm{d}, J$ 8.6, $\mathrm{Ar}), 7.35(1 \mathrm{H}, \mathrm{d}$, J 8.7, $\mathrm{Ar}), 7.73(1 \mathrm{H}, \mathrm{d}, J$ 1.9, $\mathrm{Ar}) ;{ }^{13} \mathrm{C} \mathrm{NMR}\left(125 \mathrm{MHz}, \mathrm{CDCl}_{3}\right)$ 24.9, 28.6, 34.2, 44.1, 47.3, 53.5, 55.0, 112.2, 114.3, 119.9, 120.5 (q, J 256.6), 121.3, 121.6, 127.8 (q, J 5.2), 129.4, 132.2, 137.3, 144.1, 144.8, 154.6, 154.6, 158.7; ${ }^{19}$ F NMR (470.7 $\left.\mathrm{MHz}, \mathrm{CDCl}_{3}\right)-58.2$.

\section{(S)-3-(5-Chloro-2-(4-methoxybenzyl)-1H-benzo[d]imidazol-1-yl)-N-(4-trifluoromethylphenyl)piperidine-1-} carboxamide $8\{4,5\}$.

General procedure C: $15\{4\}(1 \mathrm{~mL}, 49 \mu \mathrm{mol})$ and 4-(trifluoromethyl)phenyl isocyanate $(11 \mu \mathrm{l}, 78 \mu \mathrm{mol}) .8\{4,5\}(22$ $\mathrm{mg}, 82 \%)$, white solid; HPLC purity (as area \%) > 97; ${ }^{1} \mathrm{H} \mathrm{NMR}\left(400 \mathrm{MHz}, \mathrm{CDCl}_{3}\right) 1.52(1 \mathrm{H}, \mathrm{qt}, J 4.1$ and 13.3$), 1.72(1 \mathrm{H}$, app.d), $1.88(1 \mathrm{H}$, app.d), $2.22(1 \mathrm{H}, \mathrm{qd}, J 4.1$ and 12.8), $2.80(1 \mathrm{H}, \mathrm{td}, J 2.3$ and 13.3$), 3.39(1 \mathrm{H}$, app.t), $3.56(3 \mathrm{H}, \mathrm{s}$, $\left.\mathrm{OCH}_{3}\right), 3.70(1 \mathrm{H}, \mathrm{app} . \mathrm{d}), 4.18-4.20(3 \mathrm{H}, \mathrm{m}), 4.30(1 \mathrm{H}, \mathrm{tt}, J 4.1$ and 11.9), $6.48(1 \mathrm{H}, \mathrm{s}, \mathrm{NH}), 6.74(2 \mathrm{H}, \mathrm{d}, J 8.7, \mathrm{Ar}), 7.07$ $(2 \mathrm{H}, \mathrm{d}, J$ 8.7, $\mathrm{Ar}), 7.16(1 \mathrm{H}, \mathrm{dd}, J 2.3$ and 8.7), $7.33(1 \mathrm{H}, \mathrm{d}, J$ 8.7, $\mathrm{Ar}), 7.39(2 \mathrm{H}, \mathrm{d}, J$ 8.7, $\mathrm{Ar}), 7.50(2 \mathrm{H}, \mathrm{d}, J$ 8.7, $\mathrm{Ar}), 7.71$ $(1 \mathrm{H}, \mathrm{d}, J \mathrm{~J} 2.3, \mathrm{Ar}) ;{ }^{13} \mathrm{C}$ NMR $\left(75 \mathrm{MHz}, \mathrm{CDCl}_{3}\right)$ 25.3, 29.1, 34.7, 44.8, 47.9, 53.9, 55.6, 112.4, 114.9, 119.8, 120.4, 123.1, 126.4 (q, J 3.8), 128.3, 128.4, 129.8, 132.4, 142.3, 144.7, 154.7, 155.0, 159.4; $\left.{ }^{19} \mathrm{~F} \mathrm{NMR} \mathrm{(376.2} \mathrm{MHz,} \mathrm{CDCl}\right)-61.9$; MS $(E S I+) \mathrm{m} / \mathrm{z}=543.3$.

(S)-3-(5-Chloro-2-(4-methoxybenzyl)-1H-benzo[d]imidazol-1-yl)- $N$-phenylpiperidine-1-carboxamide 8 $\{4,6\}$.

General procedure C: $\mathbf{1 5}\{4\}(1 \mathrm{~mL}, 49.3 \mu \mathrm{mol})$ and phenyl isothocyanate $(8.1 \mu \mathrm{l}, 75.6 \mu \mathrm{mol}) .8\{4,6\}(18 \mathrm{mg}, 77 \%)$, white solid; HPLC purity (as area \%) > 99; ${ }^{1} \mathrm{H}$ NMR $\left(400 \mathrm{MHz}, \mathrm{CDCl}_{3}\right) 1.49(1 \mathrm{H}$, qt, $J 4.1$ and 13.3), 1.65,(1H, app.d), 1.86,(1H, app.d), 2.19,(1H, qd, J 4.1 and 12.8), 2.78 (1H, td, J 2.3 and 13.3), 3.35,(1H, app.t), 3.57,(3H, s, OCH $\mathrm{OCH}_{3}, 3.75$ $(1 \mathrm{H}, \mathrm{dd}, J 4.1$ and 12.8), $4.13(1 \mathrm{H}, \mathrm{app} . \mathrm{d}), 4.19(1 \mathrm{H}, \mathrm{d}, J 16.0), 4.24(1 \mathrm{H}, \mathrm{d}, J 16.0), 4.29(1 \mathrm{H}, \mathrm{tt}, J 4.6$ and 12.4$), 6.28$ $(1 \mathrm{H}, \mathrm{s}, \mathrm{NH}), 6.75(2 \mathrm{H}, \mathrm{d}, J$ 8.7, Ar), $7.10(1 \mathrm{H}, \mathrm{m}, \mathrm{Ar}), 7.08(1 \mathrm{H}, \mathrm{d}, J$ 8.7, $\mathrm{Ar}), 7.15(1 \mathrm{H}, \mathrm{dd}, J 2.3$ and 8.7), 7.25-7.26 (5H, $\mathrm{m}, \mathrm{Ar}), 7.33(1 \mathrm{H}, \mathrm{d}, \mathrm{J} 8.7, \mathrm{Ar}), 7.70(1 \mathrm{H}, \mathrm{d}, J$ 2.3, $\mathrm{Ar}) ;{ }^{13} \mathrm{C} \mathrm{NMR}\left(100 \mathrm{MHz}, \mathrm{CDCl}_{3}\right)$ 24.8, 28.6, 34.2, 44.2, 47.3, 53.4, 55.0, $112.2,114.3,119.8,120.3,122.6,123.5,127.6,127.8,128.9,129.4,132.2,138.6,144.0,154.7,155.0,158.7$; MS $(E S I+) \mathrm{m} / \mathrm{z}=475.35[\mathrm{M}+\mathrm{H}]^{+}$.

(S)-Ethyl 3-(3-(5-chloro-2-(4-methoxybenzyl)-1H-benzo[d]imidazol-1-yl)piperidine-1-carboxamido)benzoate 8\{4,7\}.

General procedure C: $15\{4\}(1 \mathrm{~mL}, 49 \mu \mathrm{mol})$ and 3-isocyanatobenzoate $(12 \mu \mathrm{l}, 76 \mu \mathrm{mol}) .8\{4,7\}(23 \mathrm{mg}, 85 \%)$, white solid; HPLC purity (as area \%) > 98; ${ }^{1} \mathrm{H}$ NMR $\left(400 \mathrm{MHz}, \mathrm{CDCl}_{3}\right) 1.33\left(3 \mathrm{H}, \mathrm{t}, J 4.1, \mathrm{CH}_{2} \mathrm{CH}_{3}\right), 1.48(1 \mathrm{H}, \mathrm{qt}, \mathrm{J} 4.1$ and 13.3$)$, $1.62(1 \mathrm{H}, \mathrm{app} . \mathrm{d}), 1.82(1 \mathrm{H}, \mathrm{app} . \mathrm{d}), 2.17(1 \mathrm{H}, \mathrm{qd}, J 4.1$ and 12.8), $2.78(1 \mathrm{H}, \mathrm{td}, J 2.3$ and 13.3), 3.36 (1H, app.t), 3.55 $\left(3 \mathrm{H}, \mathrm{s}, \mathrm{OCH}_{3}\right), 3.82(1 \mathrm{H}, \mathrm{dd}, J 4.1$ and 12.8), $4.15(1 \mathrm{H}, \mathrm{d}, J 16.0), 4.17(1 \mathrm{H}, \mathrm{app} . \mathrm{d}), 4.23(1 \mathrm{H}, \mathrm{d}, J$ 16.0), 4.25-4.33 (1H, m), $4.30\left(2 \mathrm{H}, \mathrm{q}, J\right.$ 7 7.3, $\left.\mathrm{CH}_{2} \mathrm{CH}_{3}\right), 6.70-6.73(3 \mathrm{H}, \mathrm{app} . \mathrm{d}), 7.04(2 \mathrm{H}, \mathrm{d}, J$ 8.7, $\mathrm{Ar}), 7.15$ (1H, dd, J 1.8 and 8.7), 7.28-7.33 (2H, $\mathrm{m}, \mathrm{Ar})$, 7.59-7.62 (1H, m, Ar), 7.63-7.70 (2H, m, Ar), $7.81(1 \mathrm{H}, \mathrm{app} . \mathrm{d}, \mathrm{Ar}) ;{ }^{13} \mathrm{C} \mathrm{NMR}\left(100 \mathrm{MHz}, \mathrm{CDCl}_{3}\right) 14.3,24.9,28.6$, 34.1, 44.2, 47.2, 53.4, 55.0, 61.0, 112.2, 114.2, 119.7, 121.1, 124.4, 124.8, 127.6, 127.7, 128.9, 129.4, 131.0, 132.2, 139.0, 144.0, 154.7, 154.9, 158.7, 166.3; MS (ESI+) m/z = 547.3 $[\mathrm{M}+\mathrm{H}]^{+}$. 
(S)-Ethyl 4-(3-(5-chloro-2-(4-methoxybenzyl)-1H-benzo[d]imidazol-1-yl)piperidine-1-carboxamido)benzoate 8\{4,8\}.

General procedure C: $15\{4\}(1 \mathrm{~mL}, 49.3 \mu \mathrm{mol})$ and 4-isocyanatobenzoate (14.1 mg, $75.6 \mu \mathrm{mol}) .8\{4,8\}(22 \mathrm{mg}, 82 \%)$, white solid; HPLC purity (as area \%) > 97; ${ }^{1} \mathrm{H} \mathrm{NMR}\left(400 \mathrm{MHz}, \mathrm{CDCl}_{3}\right) 1.35\left(3 \mathrm{H}, \mathrm{t}, J 7.3, \mathrm{CH}_{2} \mathrm{CH}_{3}\right), 1.50(1 \mathrm{H}, \mathrm{qt}, J 4.1$ and 13.3), 1.67 (1H, app.d), 1.86 (1H, app.d), 2.19 (1H, qd, J 4.1 and 12.8), 2.80 (1H, td, J 2.3 and 13.3), 3.37 (1H, app.t), $3.59\left(3 \mathrm{H}, \mathrm{s}, \mathrm{OCH}_{3}\right), 3.75-3.78(1 \mathrm{H}, \mathrm{m}), 4.17-4.24(3 \mathrm{H}, \mathrm{m}), 4.24-4.33(1 \mathrm{H}, \mathrm{m}), 4.33\left(2 \mathrm{H}, \mathrm{q}, J 7.3, \mathrm{CH}_{2} \mathrm{CH}_{3}\right), 6.62(1 \mathrm{H}, \mathrm{s}$, $\mathrm{NH}), 6.74(2 \mathrm{H}, \mathrm{d}, J$ 8.7, $\mathrm{Ar}), 6.75(2 \mathrm{H}, \mathrm{d}, J$ 8.7, $\mathrm{Ar}), 7.06(2 \mathrm{H}, \mathrm{d}, J$ 8.7, $\mathrm{Ar}), 7.16(1 \mathrm{H}, \mathrm{dd}, J 1.8$ and 8.7, Ar), $7.31(2 \mathrm{H}, \mathrm{d}, J$ 8.7, Ar), $7.36(1 \mathrm{H}, \mathrm{d}, J$ 8.7, $\mathrm{Ar}), 7.70\left(1 \mathrm{H}, \mathrm{d}, J\right.$ 1.8, Ar), $7.93(1 \mathrm{H}, \mathrm{d}, J$ 8.7, $\mathrm{Ar}) ;{ }^{13} \mathrm{C} \mathrm{NMR}\left(100 \mathrm{MHz}, \mathrm{CDCl}_{3}\right)$ 14.3, 24.9, 28.6, $34.2,44.2,47.2,53.4,55.1,60.7,112.2,114.3,118.6,119.8,122.7,124.9,127.7,127.7,129.4,130.6,132.1,143.0$, 144.0, 154.3, 154.6, 158.7, 166.2; MS (ESI+) $\mathrm{m} / \mathrm{z}=547.3[\mathrm{M}+\mathrm{H}]^{+}$.

(S)-3-(5-Chloro-2-(4-methoxybenzyl)-1H-benzo[d] imidazol-1-yl)-N-(4-fluorophenyl)piperidine-1-carboxamide $8\{4,9\}$.

General procedure C: $\mathbf{1 5}\{4\}(1 \mathrm{~mL}, 49.3 \mu \mathrm{mol})$ and 4-fluorophenyl isocyanate $(8.4 \mu \mathrm{l}, 75.6 \mu \mathrm{mol}) .8\{4,9\}(19 \mathrm{mg}, 78 \%)$, white solid; HPLC purity (as area \%) > 97; ${ }^{1} \mathrm{H} \mathrm{NMR}\left(400 \mathrm{MHz}, \mathrm{CDCl}_{3}\right) 1.51$ (1H, qt, J 4.1 and 13.3), 1.71 (1H, app.d), 1.86 (1H, app.d), $2.22\left(1 \mathrm{H}, \mathrm{qd}, J 4.1\right.$ and 12.8), $2.79\left(1 \mathrm{H}, \mathrm{td}, J 2.3\right.$ and 13.3), $3.38(1 \mathrm{H}, \mathrm{app} . \mathrm{t}), 3.57\left(3 \mathrm{H}, \mathrm{s}, \mathrm{OCH}_{3}\right), 3.70(1 \mathrm{H}$, app.d), $4.15(1 \mathrm{H}$, app.d), 4.21-4.34 (3H, m), $6.11(1 \mathrm{H}, \mathrm{s}, \mathrm{NH}), 6.76(2 \mathrm{H}, \mathrm{d}, J 8.7, \mathrm{Ar}), 6.96(2 \mathrm{H}, \mathrm{app} . \mathrm{t}, \mathrm{Ar}), 7.10(2 \mathrm{H}, \mathrm{d}, J$ 8.7, $\mathrm{Ar}), 7.16-7.22(3 \mathrm{H}, \mathrm{m}, \mathrm{Ar}), 7.35(1 \mathrm{H}, \mathrm{d}, J$ 8.7, $\mathrm{Ar}), 7.71(1 \mathrm{H}, \mathrm{d}, J 1.4, \mathrm{Ar}) ;{ }^{13} \mathrm{C} \mathrm{NMR}\left(100 \mathrm{MHz}, \mathrm{CDCl}_{3}\right)$ 24.8, 28.6, 34.1, 44.1, 47.3, 53.5, 55.0, 112.2, 114.3, 115.4 (d, J 22.0 Hz), 119.7, 122.4 (d, J 7.7 Hz), 122.7, 127.7, 129.4, 132.1, 134.5 (d, J 2.9), 143.9, 154.6, 155.0, 158.7, 159.1 (d, J 243.5 Hz); $\left.{ }^{19} \mathrm{~F} \mathrm{NMR} \mathrm{(376.2} \mathrm{MHz,} \mathrm{CDCl} 3\right)-119.3 ; \mathrm{MS}(\mathrm{ESI}+) \mathrm{m} / \mathrm{z}=$ $493.3[\mathrm{M}+\mathrm{H}]^{+}$.

(S)-3-(5-Chloro-2-(4-methoxybenzyl)-1H-benzo[d]imidazol-1-yl)- $N$-cyclohexylpiperidine-1-carboxamide 8\{4,10\}.

General procedure C: $15\{4\}(1 \mathrm{~mL}, 49.3 \mu \mathrm{mol})$ and cyclohexyl isocyanate $(9.4 \mu \mathrm{l}, 75.6 \mu \mathrm{mol}) .8\{4,10\}(23 \mathrm{mg}, 97 \%)$, white solid; HPLC purity (as area \%) > 96; ${ }^{1} \mathrm{H}$ NMR $\left(500 \mathrm{MHz}, \mathrm{CDCl}_{3}\right)$ 0.93-1.00 (2H, m), $1.07(1 \mathrm{H}, \mathrm{qt}, J 12.5$ and 3.5), 1.23-1.33 (2H, m), $1.36(1 \mathrm{H}, \mathrm{qt}, J 13.2$ and 4.0), $1.50(1 \mathrm{H}, \mathrm{app} . \mathrm{d}), 1.55(1 \mathrm{H}, \mathrm{dt}, J 13.0$ and 3.6), 1.62-1.65 (2H, m), 1.72 $(1 \mathrm{H}, \mathrm{app} \mathrm{dt}), 1.81-1.87(2 \mathrm{H}, \mathrm{m}), 2.08(1 \mathrm{H}, \mathrm{qd}, J 12.8$ and 4.1), $2.66(1 \mathrm{H}, \mathrm{td}, J 13.2$ and 2.5$), 3.23(1 \mathrm{H}, \mathrm{t}, J 12.1), 3.48-$ $3.56(1 \mathrm{H}, \mathrm{m}), 3.66-3.69(1 \mathrm{H}, \mathrm{m}), 3.70(3 \mathrm{H}, \mathrm{s}, \mathrm{OCH}), 3.88(1 \mathrm{H}, \mathrm{app} . \mathrm{d}), 4.03-4.05(1 \mathrm{H}, \mathrm{m}), 4.17-4.23(1 \mathrm{H}, \mathrm{m}), 4.18(1 \mathrm{H}, \mathrm{d}$, J 15.8), $4.26(1 \mathrm{H}, \mathrm{d}, J$ 15.8), $6.75(2 \mathrm{H}, \mathrm{d}, J$ 8.6, Ar), $7.05(1 \mathrm{H}, \mathrm{d}, J$ 8.6, $\mathrm{Ar}), 7.10(1 \mathrm{H}, \mathrm{dd}, J 8.7$ and 2.0, Ar), $7.29(1 \mathrm{H}, \mathrm{d}, J$ 8.7, $\mathrm{Ar}), 7.67(1 \mathrm{H}, \mathrm{d}, J 2.0, \mathrm{Ar}) ;{ }^{13} \mathrm{C}$ NMR $\left(125 \mathrm{MHz}, \mathrm{CDCl}_{3}\right)$ 24.7, 25.1, 25.1, 25.5, 28.6, 33.8, 33.8, 34.2, 44.1, 47.1, $49.6,53.4,55.2,112.3,114.3,119.7,122.5,127.6,127.9,129.4,132.2,154.6,156.9,158.9 ; \mathrm{MS}(\mathrm{ESI}+) \mathrm{m} / \mathrm{z}=481.4$ $[\mathrm{M}+\mathrm{H}]^{+}$.

(S)-N-Benzyl-3-(2-benzyl-5-chloro-1H-benzo[d]imidazol-1-yl)piperidine-1-carbothioamide 16.

General procedure C: $15\{1\}$ (16 mg, $49.2 \mu \mathrm{mol}$ ) and benzyl isothiocyanate $(7.2 \mu \mathrm{l}, 54.1 \mu \mathrm{mol}) .16(15 \mathrm{mg}, 64 \%)$ as a white solid; HPLC purity (as area \%) > 93; ${ }^{1} \mathrm{H}$ NMR (500 MHz, CDCl $) 1.43(1 \mathrm{H}, \mathrm{qt}, J 4.1$ and 13.3), 1.55 (1H, app.d), 1.77-1.80 (1H, m), $2.25(1 \mathrm{H}, \mathrm{qd}, J 12.8$ and 4.4), $3.03(1 \mathrm{H}, \mathrm{td}, J 13.4$ and 2.4), $3.50(1 \mathrm{H}, \mathrm{app} . \mathrm{t}), 4.35-4.46(4 \mathrm{H}, \mathrm{m}), 4.67-$ $4.71(1 \mathrm{H}, \mathrm{m}), 4.74(1 \mathrm{H}, \mathrm{dd}, J 14.6$ and 4.6), $4.97(1 \mathrm{H}, \mathrm{dd}, J 14.6$ and 5.4), $5.65(1 \mathrm{H}$, app.t, NH), 7.15-7.22 (6H, m, Ar), 7.30-7.36 (6H, m, Ar), $7.73\left(1 \mathrm{H}, \mathrm{d}, J\right.$ 2.0, Ar); ${ }^{13} \mathrm{C} N M R\left(125 \mathrm{MHz}, \mathrm{CDCl}_{3}\right)$ 24.5, 28.4, 35.0, 48.0, 50.4, 51.3, 52.7, 112.0, $119.9,122.7,127.2,127.7,127.8,127.9,128.4,128.8,128.9,132.1,136.2,137.8,144.1,154.5,183.2$. LC-MS (ESI+) $\mathrm{m} / \mathrm{z}=475.3[\mathrm{M}+\mathrm{H}]^{+}$.

(S)- 2-Benzyl-1-(1-(benzylsulfonyl)piperidin-3-yl)-5-chloro-1H-benzo[d]imidazole 17. 
Phenylmethanesulfonyl chloride (12 mg, $60.9 \mu \mathrm{mol}, 1.1 \mathrm{eq}$ ) was added to a solution of 15 (18 mg, $55.4 \mu \mathrm{mol}, 1 \mathrm{eq})$ and triethylamine $(11.6 \mu \mathrm{l}, 83.1 \mu \mathrm{mol}, 1.5 \mathrm{eq})$ in $\mathrm{DCM}(1 \mathrm{~mL})$. The mixture was stirred at RT for $1 \mathrm{~h}$, then quenched with water $(3 \mathrm{~mL})$. The organic layer was separated, dried $\left(\mathrm{MgSO}_{4}\right)$, and evaporated. Chromatography (5\% DCM, methanol) gave 17 (25 mg, 94\%) as a white solid; HPLC purity (as area \%) > 96; ${ }^{1} \mathrm{H} \mathrm{NMR}\left(500 \mathrm{MHz}, \mathrm{CDCl}_{3}\right)$ 1.34-1.41 $(2 \mathrm{H}, \mathrm{m}), 1.64-1.68(1 \mathrm{H}, \mathrm{m}), 1.94(1 \mathrm{H}, \mathrm{qd}, J 12.6$ and 4.2), $2.54(1 \mathrm{H}, \mathrm{td}, J 12.6$ and 2.6), $3.01(1 \mathrm{H}, \mathrm{app} . \mathrm{t}), 3.54(1 \mathrm{H}, \mathrm{dd}, J$ 12.3 and 4.6), 3.61-3.64 (1H, m), $4.13(1 \mathrm{H}, \mathrm{d}, J 14.1), 4.17(1 \mathrm{H}, \mathrm{d}, J 14.1), 4.19(1 \mathrm{H}, \mathrm{d}, J 15.9), 4.24(1 \mathrm{H} ; \mathrm{tt}, J 12.0$ and 4.1), $4.40(1 \mathrm{H}, \mathrm{d}, J 15.9), 7.11(1 \mathrm{H}, \mathrm{d}, J$ 8.7, Ar), $7.14(1 \mathrm{H}, \mathrm{dd}, J 8.7$ and 1.8, Ar), 7.18-7.23 (3H, m, Ar), 7.27-7.30 (3H, $m, \operatorname{Ar}), 7.32-7.37(4 \mathrm{H}, \mathrm{m}, \mathrm{Ar}), 7.72\left(1 \mathrm{H}, \mathrm{d}, J\right.$ 1.8, Ar); LC-MS (ESI+) m/z $=480.3[\mathrm{M}+\mathrm{H}]^{+}$.

\section{General Procedure D:}

A mixture of 4-chloro-2-fluoronitrobenzene $(1.0 \mathrm{~g}, 5.7 \mathrm{mmol})$, the required benzylamine $(5 \mathrm{eq})$, potassium carbonate $(1.97 \mathrm{~g}, 10.66 \mathrm{mmol})$ and ethanol $(20 \mathrm{~mL})$ was heated at $120^{\circ} \mathrm{C}$ for $25 \mathrm{~min}$ in a microwave vial, then filtered and concentrated. The residue was diluted with ethylacetate $(20 \mathrm{~mL})$ washed with $\mathrm{HCl}(1 \mathrm{~N}, 20 \mathrm{~mL})$, water $(20 \mathrm{~mL})$ and brine $(20 \mathrm{~mL})$, dried $\left(\mathrm{MgSO}_{4}\right)$ and concentrated. Recrystallisation from absolute ethanol gave 19 as bright orangeyellow crystals.(45)

\section{N-Benzyl-5-chloro-2-nitroaniline 19\{1\} (52)}

General Procedure D: 4-chloro-2-fluoronitrobenzene (1.0 g, $5.7 \mathrm{mmol})$, benzylamine (2.79 mL, $25.6 \mathrm{mmol}) .19\{1\} 66$ $\% ;{ }^{1} \mathrm{H}$ NMR (300 MHz , $\left.\mathrm{CDCl}_{3}\right) \delta \mathrm{ppm} 4.61$ (d, J 5.3, 2H, $\left.\mathrm{CH}_{2}-\mathrm{N}\right), 6-8(\mathrm{~m}, 7 \mathrm{H}, \mathrm{Ar}-\mathrm{H})$.

\section{5-Chloro-N-(3-chlorobenzyl)-2-nitroaniline 19\{2\}}

General Procedure D: 4-chloro-2-fluoronitrobenzene (1.0 g, $5.7 \mathrm{mmol})$, 3-chlorobenzylamine $(2.79 \mathrm{~mL}, 22.8 \mathrm{mmol})$. 19\{2\} $82 \% ;{ }^{1} \mathrm{H} \mathrm{NMR}\left(300 \mathrm{MHz}, \mathrm{CDCl}_{3}\right) \delta \mathrm{ppm} 3.82\left(\mathrm{~s}, 3 \mathrm{H}, \mathrm{OCH}_{3}\right), 4.51\left(\mathrm{~d}, J\right.$ 5.7, 2H, $\left.\mathrm{CH}_{2}-\mathrm{N}\right), 6.61(\mathrm{~d}, \mathrm{br}, J \mathrm{~J} .3,1 \mathrm{H}, \mathrm{Ar}-\mathrm{H})$, $6.84(\mathrm{~m}, 5 \mathrm{H}, \mathrm{Ar}-\mathrm{H}), 8.11$ (d, J 8.1, 1H, Ar-H), $8.46(\mathrm{~s}, 1 \mathrm{H}, \mathrm{N}-\mathrm{H})$.

\section{5-Chloro-N-(3-methoxybenzyl)-2-nitroaniline 19\{3\}}

General Procedure D: 4-chloro-2-fluoronitrobenzene (1.0g, $5.7 \mathrm{mmol})$, 3-methoxybenzylamine (2.9 7mL, 22.8 mmol). 19\{3\} $82 \%$; ${ }^{1} \mathrm{H} \mathrm{NMR}\left(300 \mathrm{MHz}, \mathrm{CDCl}_{3}\right) 3.82\left(\mathrm{~s}, 3 \mathrm{H}, \mathrm{OCH}_{3}\right), 4.51$ (d, J 5.7, 2H, $\left.\mathrm{CH}_{2}-\mathrm{N}\right), 6.61$ (d, br, J 7.3,1H, Ar-H), $6.84(\mathrm{~m}, 5 \mathrm{H}, \mathrm{Ar}-\mathrm{H}), 8.11(\mathrm{~d}, \mathrm{~J} 8.1,1 \mathrm{H}, \mathrm{Ar}-\mathrm{H}), 8.46(\mathrm{~s}, 1 \mathrm{H}, \mathrm{N}-\mathrm{H})$.

\section{5-Chloro-N-(4-methoxybenzyl)-2-nitroaniline 19\{4\}(53)}

General Procedure D: 4-chloro-2-fluoronitrobenzene (1.0g, $5.7 \mathrm{mmol})$, 4-methoxybenzylamine (2.97mL, 22.8mmol), $19\{4\} 82 \% ;{ }^{1} \mathrm{H} \mathrm{NMR}\left(300 \mathrm{MHz}, \mathrm{CDCl}_{3}\right) \delta \mathrm{ppm} 3.73\left(\mathrm{~s}, 3 \mathrm{H}, \mathrm{OCH}_{3}\right), 4.51\left(\mathrm{~d}, J 5.7,2 \mathrm{H}, \mathrm{CH}_{2}-\mathrm{N}\right), 6.61(\mathrm{~d}, \mathrm{br}, J$ 7.3, $1 \mathrm{H}, \mathrm{Ar}-\mathrm{H})$, $6.81(\mathrm{~s}, 1 \mathrm{H}, \mathrm{Ar}-\mathrm{H}), 6.92(\mathrm{~d}, J$ 8 8.2, Ar-H), $8.11(\mathrm{~d}, J$ 8.1, 1H, Ar-H), $8.36(\mathrm{~s}, 1 \mathrm{H}, \mathrm{N}-\mathrm{H})$.

\section{General Procedure E: Benzimidazole Intermediate Synthesis (20)}

A mixture 18 (0.3g, $1.41 \mathrm{mmol})$, sodium dithionite $(0.67 \mathrm{~g}, 3.84 \mathrm{mmol})$ and 19 (1.0 eq.) in methanol $(16 \mathrm{~mL})$ and water $(4 \mathrm{~mL})$ was heated by microwave for 10 mins at $100{ }^{\circ} \mathrm{C}$, then diluted with ethyl acetate $(20 \mathrm{~mL})$, washed with water $(20 \mathrm{~mL})$ and brine $(20 \mathrm{~mL})$, dried $\left(\mathrm{MgSO}_{4}\right)$ and evaporatated. Recrystallisation from ethyl acetate, petrol gave 20. 
4-(1-Benzyl-6-chloro-1H-benzoimidazole-2-yl)piperidine-1-carboxylic acid tert-butyl ester 20\{1\}

General Procedure E: $19\{1\}(0.32 \mathrm{~g}, 1.22 \mathrm{mmol})$, sodium dithionite $(0.64 \mathrm{~g}, 3.66 \mathrm{mmol}), \mathbf{1 8}(0.29 \mathrm{~g}, 1.34 \mathrm{mmol}) .20\{1\}$ 77\%; mp 157-158 ${ }^{\circ} \mathrm{C}$; HPLC purity (as area \%) > 96; ${ }^{1} \mathrm{H} \mathrm{NMR}\left(400 \mathrm{MHz}, \mathrm{CDCl}_{3}\right) 1.44(\mathrm{~s}, 9 \mathrm{H}, \mathrm{tBu}), 1.76\left(\mathrm{~m}, \mathrm{br}, 2 \mathrm{H}, \mathrm{CH}_{2^{-}}\right.$ $\left.\mathrm{CH}), 1.99\left(\mathrm{~m}, \mathrm{br}, 2 \mathrm{H}, \mathrm{CH}_{2}-\mathrm{CH}\right), 2.73\left(\mathrm{~m}, \mathrm{br} 2 \mathrm{H}, \mathrm{CH}_{2}-\mathrm{N}\right), 2.92(\mathrm{~m} \mathrm{br}, 1 \mathrm{H}, \mathrm{CH} \mathrm{CH})^{2}\right), 4.25\left(\mathrm{~m}, \mathrm{br}, 2 \mathrm{H}, \mathrm{CH}_{2} \mathrm{~N}\right), 5.32\left(\mathrm{~s}, 2 \mathrm{H} \mathrm{CH}_{2}\right.$ N), 6.99 (d, 1H, J 1.7, Ar-H), 7.17 (s, 1H, Ar-H), 7.18 (m, 5H, Ar-H), 7.66 (d, 1H, 2.2 Hz, Ar-H); LC-MS (ESI+) m/z 426 $[\mathrm{M}+\mathrm{H}]^{+} ;$IR 732, 819, 837, 1022, 1168.26, 1236, 1412, $2924 \mathrm{~cm}^{-1}$.

tert-Butyl 4-(6-chloro-1-(4-chlorobenzyl)-1H-benzo[d]imidazol-2-yl)piperidine-1-carboxylate 20\{2\}

General Procedure E: 19\{2\} (0.15g, $0.51 \mathrm{mmol})$, sodium dithionite $(0.268 \mathrm{~g}, 1.54 \mathrm{mmol}), 18(0.12 \mathrm{~g}, 0.56 \mathrm{mmol}) .20\{2\}$ 90\%; mp 139-141 ${ }^{\circ} \mathrm{C}$; HPLC purity (as area \%) > 97; ${ }^{1} \mathrm{H}$ NMR (400 MHz, $\left.\mathrm{CDCl}_{3}\right) \delta \mathrm{ppm}: 1.43(\mathrm{~s}, 9 \mathrm{H}, \mathrm{tBu}), 1.76(\mathrm{~m}, \mathrm{br}, 2 \mathrm{H}$, $\left.\mathrm{CH}_{2}-\mathrm{CH}\right), 1.98\left(\mathrm{~m}, \mathrm{br}, 2 \mathrm{H}, \mathrm{CH}_{2}-\mathrm{CH}\right), 2.72\left(\mathrm{~m}, \mathrm{br} 2 \mathrm{H}, \mathrm{CH}_{2}-\mathrm{N}\right), 2.93\left(\mathrm{~m} \mathrm{br}, 1 \mathrm{H}, \mathrm{CH} \mathrm{CH}_{2}\right), 4.22\left(\mathrm{~m}, \mathrm{br}, 2 \mathrm{H}, \mathrm{CH}_{2} \mathrm{~N}\right), 5.29(\mathrm{~s}, 2 \mathrm{H}$ $\left.\mathrm{CH}_{2} \mathrm{~N}\right), 6.70(\mathrm{~d}, 1 \mathrm{H}, J$ 1.8, Ar-H), $6.99(\mathrm{~s}, 1 \mathrm{H}, \mathrm{Ar}-\mathrm{H}), 7.19$ (s, 1H, Ar-H), 7.25 (m, 3H, Ar-H), 7.61 (d, 1H, J 2.3, Ar-H); LCMS (ESI+) m/z $461[\mathrm{M}+\mathrm{H}]^{+} ;$IR 773, 1157, 1237, 1410, 1670, 2018, 2359, $2964 \mathrm{~cm}^{-1}$.

tert-Butyl 4-(6-chloro-1-(3-methoxybenzyl)-1H-benzo[d]imidazol-2-yl)piperidine-1-carboxylate 20\{3\}

General Procedure E: $19\{3\}$ (0.36g, 1.23mmol), sodium dithionite (0.71g, $4.05 \mathrm{mmol}), 18$ (0.29g, 1.35mmol). 20\{3\} 73\%; mp 142-143 ${ }^{\circ} \mathrm{C}$; HPLC purity (as area \%) > 97; ${ }^{1} \mathrm{H} \mathrm{NMR}\left(400 \mathrm{MHz}, \mathrm{CDCl}_{3}\right) 1.43(\mathrm{~s}, 9 \mathrm{H}, \mathrm{tBu}), 1.77\left(\mathrm{~m}, \mathrm{br}, 2 \mathrm{H}, \mathrm{CH}_{2-}\right.$ $\mathrm{CH}), 1.97\left(\mathrm{~m}, \mathrm{br}, 2 \mathrm{H}, \mathrm{CH}_{2}-\mathrm{CH}\right), 2.71\left(\mathrm{~m}, \mathrm{br} 2 \mathrm{H}, \mathrm{CH}_{2}-\mathrm{N}, 2.91\left(\mathrm{~m}, \mathrm{br}, 1 \mathrm{H}, \mathrm{CH} \mathrm{CH}_{2}\right), 3.72\left(\mathrm{~s}, 3 \mathrm{H}, \mathrm{OCH}_{3}\right) 4.22(\mathrm{~m}, \mathrm{br}, 2 \mathrm{H}\right.$, $\left.\mathrm{CH}_{2} \mathrm{~N}\right), 5.28\left(\mathrm{~s}, 2 \mathrm{H} \mathrm{CH} \mathrm{CH}_{2} \mathrm{~N}\right), 6.51(\mathrm{~s}, 1 \mathrm{H}, \mathrm{Ar}-\mathrm{H}), 6.53(\mathrm{~d}, 1 \mathrm{H}, 1.8 \mathrm{~Hz} \mathrm{Ar}-\mathrm{H}), 6.77$ (d, 1H, $\left.1.72 \mathrm{~Hz}, \mathrm{Ar}-\mathrm{H}\right), 7.19(\mathrm{~s}, 1 \mathrm{H}, \mathrm{Ar}-\mathrm{H} \mathrm{Cl})$, 7.22 (m, 1H, Ar-H), 7.24 (d, 1H, Ar-H), 7.67 (d, 2.2 Hz, 1H, Ar-H); LC-MS (ESI+) m/z 456 [M+H]+; IR 821, 1043, 1398, $1693,2160,2970 \mathrm{~cm}^{-1}$.

tert-Butyl 4-(6-chloro-1-(4-methoxybenzyl)-1H-benzo[d]imidazol-2-yl)piperidine-1-carboxylate. 20\{4\}

General Procedure E: $19\{4\}$ (0.29g, 1.0mmol), sodium dithionite (0.52g, $3.0 \mathrm{mmol}), 18$ (0.23g, $1.1 \mathrm{mmol}) .20\{4\} 90 \%$; mp 142-143 ${ }^{\circ} \mathrm{C}$; HPLC purity (as area \%) > 91; ${ }^{1} \mathrm{H} N M R\left(400 \mathrm{MHz}, \mathrm{CDCl}_{3}\right) 1.39(\mathrm{~s}, 9 \mathrm{H}, \mathrm{tBu}), 1.61\left(\mathrm{~m}, \mathrm{br}, 2 \mathrm{H}, \mathrm{CH}_{2}-\mathrm{CH}\right)$, $1.82\left(\mathrm{~m}, \mathrm{br}, 2 \mathrm{H}, \mathrm{CH}_{2}-\mathrm{CH}\right), 2.70\left(\mathrm{~m}, \mathrm{br} 2 \mathrm{H}, \mathrm{CH}_{2}-\mathrm{N}\right), 2.32(\mathrm{~m} \mathrm{br}, 1 \mathrm{H}, \mathrm{CH} \mathrm{CH}), 3.69\left(\mathrm{~s}, 3 \mathrm{H}, \mathrm{OCH}_{3}\right) 4.01\left(\mathrm{~m}, \mathrm{br}, 2 \mathrm{H}, \mathrm{CH}_{2} \mathrm{~N}\right)$, $5.46\left(\mathrm{~s}, 2 \mathrm{H} \mathrm{CH} \mathrm{H}_{2} \mathrm{~N}\right), 6.86(\mathrm{~d}, J 3,2 \mathrm{H}, \mathrm{Ar}-\mathrm{H}), 7.10(\mathrm{~d}, 2 \mathrm{H}, J$ 2.8, Ar-H), $7.15(\mathrm{~d}, J 3.1,1 \mathrm{H}, \mathrm{Ar}-\mathrm{H}), 7.58(\mathrm{~d}, J 2.9,1 \mathrm{H}, \mathrm{Ar}-\mathrm{H})$, 7.60 (s, 1H, Ar-H); LC-MS (ESI+) m/z $456[\mathrm{M}+\mathrm{H}]^{+}$; IR U $\max 809,1030,1236,1416,1682,2163,2927 \mathrm{~cm}^{-1}$.

\section{General Procedure F: Boc deprotection}

A mixture of $20(0.40 \mathrm{~g}, 0.94 \mathrm{mmol})$, TFA $(0.6 \mathrm{~mL})$ and DCM $(2 \mathrm{~mL})$ was stirred at $\mathrm{rt}$ for $4.5 \mathrm{~h}$, then concentrated and diluted with methanol $(4 \mathrm{~mL}) . \mathrm{K}_{2} \mathrm{CO}_{3}(0.2 \mathrm{~g})$ was added and the suspension was stirred at $\mathrm{rt}$, then filtered, and concentrated. Chromatography ( $5 \% \mathrm{DCM}, \mathrm{MeOH}$ ) gave $\mathbf{2 1}$. Intermediates $\mathbf{2 1}\{2\}, \mathbf{2 1}\{3\}, \mathbf{2 1}\{4\}$ were used directly in the subsequent step without purification.

\section{1-Benzyl-6-chloro-2-(piperidin-4-yl)-1H-benzo[d]imidazole 21\{1\}}

A mixture of $20\{1\}(0.40 \mathrm{~g}, 0.94 \mathrm{mmol})$, TFA $(0.6 \mathrm{~mL})$ and $\mathrm{DCM}(2 \mathrm{~mL})$ was stirred at $\mathrm{rt}$ for $4.5 \mathrm{~h}$, then concentrated and diluted with methanol $(4 \mathrm{~mL}) \cdot \mathrm{K}_{2} \mathrm{CO}_{3}(200 \mathrm{mg})$ was added and the suspension was stirred at $\mathrm{rt}$, then filtered, and concentrated. Chromatography (5\% DCM, MeOH) gave 21\{1\} 72\%; HPLC purity (as area \%) > 98; ${ }^{1} \mathrm{H}$ NMR (400 MHz ,CDCl $\left.{ }_{3}\right) 1.62$ (s. br. $\left.1 \mathrm{H}, \mathrm{N}-\mathrm{H}\right) 1.71$ (m, br, 2H, $\left.\mathrm{CH}_{2} \mathrm{CH}_{2}-\mathrm{NH}\right), 1.82\left(\mathrm{~m}, 2 \mathrm{H}, \mathrm{CH}_{2} \mathrm{CH}_{2}-\mathrm{NH}\right), 2.61\left(\mathrm{~m}, 2 \mathrm{H}, \mathrm{CH}_{2}-\mathrm{CH}_{2}-\mathrm{NH}\right), 2.82(\mathrm{~m}$, $\left.1 \mathrm{H}, \mathrm{CHCH}_{2}\right), 3.18\left(\mathrm{~d}, \mathrm{br}, 2 \mathrm{H}, J\right.$ 11.4, $\left.\mathrm{CH}_{2} \mathrm{CH}_{2}-\mathrm{NH}\right), 5.21\left(\mathrm{~s}, 2 \mathrm{H}, \mathrm{CH}_{2}-\mathrm{N}\right), 6.98(\mathrm{~m}, 2 \mathrm{H}, J$ 1.4, 7.5, Ar-H), $7.09(\mathrm{~m}, 1 \mathrm{H}, J$ 1.7, Ar-H), 7.12 (dd, 1H, J 1.91, 8.63, Ar-H), 7.25 (m, 3H, Ar-H), 7.60 (d, 1H, J 8.71, ArH); LC-MS (ESI+) m/z 326 [M+H] . 


\section{General Procedure G: Benzimidazole Synthesis (9)}

$21(0.01 \mathrm{~g}, 0.028 \mathrm{mmol})$ was added to a solution of the appropriate isocyanate (1.2 eq.) in DCM (2 mL) and the mixture was stirred at $\mathrm{rt}$ for $1 \mathrm{~h}$, then concentrated. Chromatography ( $\mathrm{NH}$-silica; $2 \% \mathrm{MeOH}, \mathrm{DCM}$ ) gave 9 as glassy solids.

Ethyl-4-(1-benzyl-6-chloro-1H-benzo[d]imidazole-2-yl)piperidine-1-carboxamido)benzoate $9\{1,2\}$

General Procedure G: $21\{1\}(0.008 \mathrm{~g}, 0.028 \mathrm{mmol})$, 4-acetylphenylisocyanate $(0.005 \mathrm{~g}, 0.031 \mathrm{mmol}) .9\{1,2\} 56 \%$; HPLC purity (as area \%) > 99; ${ }^{1} \mathrm{H} N M R\left(400 \mathrm{MHz}, \mathrm{CDCl}_{3}\right) 1.89\left(\mathrm{~m}, 2 \mathrm{H}, \mathrm{CH}_{2} \mathrm{CH}\right), 2.05\left(\mathrm{~m}, 2 \mathrm{H}, \mathrm{CH}_{2} \mathrm{NCO}\right), 2.48\left(\mathrm{~s}, 3 \mathrm{H}, \mathrm{CH}_{3}\right)$ $3.04\left(\mathrm{~m}, 2 \mathrm{H}, \mathrm{CH}_{2} \mathrm{NCO}\right), 3.08\left(\mathrm{~m}, 1 \mathrm{H}, \mathrm{CH}_{2} \mathrm{CH}\right), 4.19\left(\mathrm{~m}, 2 \mathrm{H}, \mathrm{CH}_{2} \mathrm{CH}_{2} \mathrm{NCO}\right), 5.35$ (s, 2H, $\left.\mathrm{CH}_{2} \mathrm{Ar}\right), 6.58(\mathrm{~s}, 1 \mathrm{H}, \mathrm{N}-\mathrm{H}), 7.05$ (s, $1 \mathrm{H}, \operatorname{Ar}-\mathrm{H}), 7.08(\mathrm{~d}, 1 \mathrm{H}, J 1.8 \mathrm{~Hz}, \mathrm{Ar}-\mathrm{H}), 7.26(\mathrm{~m}, 5 \mathrm{H}, \mathrm{Ar}-\mathrm{H}), 7.48$ (d, 2H, J $8.91 \mathrm{~Hz}, \operatorname{Ar}-\mathrm{H}), 7.68(\mathrm{~d}, 1 \mathrm{H}, J 7.76 \mathrm{~Hz}, \operatorname{Ar}-\mathrm{H})$, $7.91(\mathrm{~d}, 2 \mathrm{H}, J 8.87 \mathrm{~Hz}, \mathrm{Ar}-\mathrm{H})$ LC-MS (ESI+) $m / z=485[\mathrm{M}+\mathrm{H}]^{+}$.

\section{Ethyl-4-(1-benzyl-6-chloro-1H-benzo[d]imidazole-2-yl)piperidine-1-carboxamido)benzoate 9\{1,8\}}

General Procedure G: $21\{1\}$ (0.012 g, $0.037 \mathrm{mmol})$, ethyl 4-isocyanobenzoate (0.008 g, 0.041mmol). 9\{1,8\} 8\%; HPLC purity (as area \%) > 99; ${ }^{1} \mathrm{H} \mathrm{NMR}\left(400 \mathrm{MHz}, \mathrm{CDCl}_{3}\right) 1.40\left(\mathrm{t}, 3 \mathrm{H}, J 7.1 \mathrm{~Hz}, \mathrm{OCH}_{2} \mathrm{CH}_{3}\right), 1.89(\mathrm{~m}, 2 \mathrm{H}, \mathrm{CH} 2 \mathrm{CH}), 2.05(\mathrm{~m}, 2 \mathrm{H}$, $\left.\mathrm{CH}_{2} \mathrm{NCO}\right), 3.04\left(\mathrm{~m}, 2 \mathrm{H}, \mathrm{CH}_{2} \mathrm{NCO}\right), 3.08\left(\mathrm{~m}, 1 \mathrm{H}, \mathrm{CH}_{2} \mathrm{CH}\right), 4.19\left(\mathrm{~m}, 2 \mathrm{H}, \mathrm{CH}_{2} \mathrm{CH}_{2} \mathrm{NCO}\right), 4.37$ (q, 2H, J $\left.7.12 \mathrm{~Hz}, \mathrm{OCH}_{2} \mathrm{CH}_{3}\right)$, $5.35\left(\mathrm{~s}, 2 \mathrm{H}, \mathrm{CH}_{2} \mathrm{Ar}\right), 6.58(\mathrm{~s}, 1 \mathrm{H}, \mathrm{N}-\mathrm{H}), 7.05$ (s, 1H, J $\left.1.4 \mathrm{~Hz}, \mathrm{Ar}-\mathrm{H}\right), 7.08$ (d, 1H, J 1.8 Hz, Ar-H), 7.26 (m, 5H, Ar-H), 7.48 (d, $2 \mathrm{H}, J 8.9 \mathrm{~Hz}, \mathrm{Ar}-\mathrm{H}), 7.68$ (d, 1H, J 7.7 Hz, Ar-H), 8.01 (d, 2H, J $8.9 \mathrm{~Hz}, \mathrm{Ar}-\mathrm{H}$ ); LC-MS (ESI+) m/z = 517 [M+H] .

\section{4-(1-Benzyl-6-chloro-1H-benzo[d]imidazol-2-yl)- $\mathrm{N}$-(4-fluorophenyl)piperidine-1-carboxamide $9\{1,9\}$}

General Procedure G: $21\{1\}$ (0.009 g, $0.028 \mathrm{mmol}$ ) was added to a stirred solution of 4-fluorophenylisocyanate (3.73 $\mu \mathrm{L}, 0.033 \mathrm{mmol}$ ). 9\{1,9\} $30 \%$; HPLC purity (as area \%) > 92; ${ }^{1} \mathrm{H} \mathrm{NMR}\left(400 \mathrm{MHz}, \mathrm{CDCl}_{3}\right) 1.79\left(\mathrm{~m}, 2 \mathrm{H}, \mathrm{CH}_{2} \mathrm{CH}_{2}-\mathrm{NH}\right), 1.97$ (m, 2H, $\left.\mathrm{CH}_{2} \mathrm{CH}_{2}-\mathrm{NH}\right), 2.91\left(\mathrm{~m}, 3 \mathrm{H}, \mathrm{CH}_{2}-\mathrm{CH}_{2}-\mathrm{NH}, \mathrm{CH}-\mathrm{CH}_{2}\right), 4.12\left(\mathrm{~m}, 2 \mathrm{H}, \mathrm{CH}_{2}-\mathrm{N}\right), 5.22\left(\mathrm{~s}, 2 \mathrm{H}, \mathrm{CH}_{2}-\mathrm{N}\right), 6.38(\mathrm{~s}, 1 \mathrm{H}, \mathrm{N}-\mathrm{H})$, $6.89(\mathrm{~m}, 2 \mathrm{H}, \mathrm{Ar}-\mathrm{H}-\mathrm{F}), 6.87$ (s, 1H, Ar-H), 6.92 (br d, 1H, J 1.94, Ar-H), 7.15 (br s, 1H, Ar-H), 7.19 (m, 2H, , Ar-H), 7.25 (m, 5H, Ar-H), 7.60 (d, 1H, $8.7 \mathrm{~Hz}, \mathrm{Ar}-\mathrm{H})$.; LC-MS (ESI+) m/z $463[\mathrm{M}+\mathrm{H}]^{+}$.

\section{4-(1-Benzyl-6-chloro-1H-benzo[d]imidazole-2-yl)-N-cyclohexylpiperidine-1-carboxamide $\mathbf{9}\{1,10\}$}

General Procedure G: 21\{1\} (0.011g, 0.032mmol), cyclohexylisocyanate $(0.004 \mathrm{~g}, 0.036 \mathrm{mmol}) .9\{1,10\}$ 68\%; HPLC purity (as area \%) > 98; ${ }^{1} \mathrm{H}$ NMR $\left(400 \mathrm{MHz}, \mathrm{CDCl}_{3}\right) 1.09\left(\mathrm{~m}, 3 \mathrm{H}\right.$, cyclohexyl), $1.28\left(\mathrm{~m}, 2 \mathrm{H}, \mathrm{CH}_{2} \mathrm{CH}-\mathrm{NH}\right), 1.52(\mathrm{~m}, 1 \mathrm{H}$, cyclohexyl), $1.62\left(\mathrm{~m}, 2 \mathrm{H}\right.$, cyclohexyl), $1.71\left(\mathrm{~m}, 2 \mathrm{H}, \mathrm{CH}_{2}-\mathrm{CH}_{2}-\mathrm{N}\right), 1.85(\mathrm{~m}, 2 \mathrm{H}$, cyclohexyl), 1.91 ( $\mathrm{m}, 2 \mathrm{H}$, cyclohexyl), 2.74 (m, 2H, CH2-NCO), 2.85 (m, 1H, NH-CH $), 3.54(\mathrm{~m}, 1 \mathrm{H}) 3.93$ (br d, 2H, J 14.59, $\left.\mathrm{CH}_{2} \mathrm{CH}\right), 4.19$ (br d, 1H, N-H), 6.85 (m, $1 \mathrm{H}, \mathrm{Ar}-\mathrm{H}), 6.87$ (d, 1H, J 2.27 ), $7.12(\mathrm{~m}, 1 \mathrm{H}, \mathrm{Ar}-\mathrm{H}), 7.19$ (dd, 1H, J 2.20, 7.89, Ar-H), 7.25 (m, 3H, Ar-H), 7.61 (d, 1H, $9.39 \mathrm{~Hz}, \mathrm{Ar}-\mathrm{H}) . ;$ LC-MS (ES+) $m / z 451[\mathrm{M}+\mathrm{H}]^{+}$.

$\mathbf{N}$-(4-Acetylphenyl)-4-(6-chloro-1-(3-chlorobenzyl)-1H-benzo[d] imidazole-2-yl)piperidine-1-carboxamide 9\{2,2\}

General Procedure G: $21\{2\}(0.011 \mathrm{~g}, 0.029 \mathrm{mmol})$, 4-acetylphenylisocyanate $(0.005 \mathrm{~g}, 0.032 \mathrm{mmol}) .9\{2,2\}$ 81\%; HPLC purity (as area \%) > 99; ${ }^{1} \mathrm{H} N M R\left(400 \mathrm{MHz}, \mathrm{CDCl}_{3}\right) 1.88\left(\mathrm{~m}, 2 \mathrm{H}, \mathrm{CH}_{2} \mathrm{CH}\right), 2.09\left(\mathrm{~m}, 2 \mathrm{H}, \mathrm{CH}_{2} \mathrm{NCO}\right), 2.49,\left(\mathrm{~s}, 3 \mathrm{H}, \mathrm{COCH}_{3}\right)$ $2.99\left(\mathrm{~m}, 2 \mathrm{H}, \mathrm{CH}_{2} \mathrm{NCO}\right), 3.07\left(\mathrm{~m}, 1 \mathrm{H}, \mathrm{CH}_{2} \mathrm{CH}\right), 4.17\left(\mathrm{~m}, 2 \mathrm{H}, \mathrm{CH}_{2} \mathrm{CH}_{2} \mathrm{NCO}\right), 5.31\left(\mathrm{~s}, 2 \mathrm{H}, \mathrm{CH}_{2} \mathrm{Ar}\right), 6.49(\mathrm{~s}, 1 \mathrm{H}, \mathrm{N}-\mathrm{H}), 6.85(\mathrm{~m}$, $1 \mathrm{H}, \operatorname{Ar}-\mathrm{H}), 7.06$ (s, 1H, Ar-H), 7.14 (d, 2H, J $8.37 \mathrm{~Hz}, \mathrm{Ar}-\mathrm{H}) 7.17$ (d, 1H, J $1.82 \mathrm{~Hz}, \mathrm{Ar}-\mathrm{H}), 7.39$ (d, 3H, J $7.91 \mathrm{~Hz} \mathrm{Ar}-\mathrm{H})$, $7.38(\mathrm{~d}, 2 \mathrm{H}, J 8.85 \mathrm{~Hz}, \mathrm{Ar}-\mathrm{H}), 7.81(\mathrm{~d}, 1 \mathrm{H}, J 8.56 \mathrm{~Hz}, \mathrm{Ar}-\mathrm{H})$; LC-MS (ESI+) $m / z=519[\mathrm{M}+\mathrm{H}]^{+}$.

4-(6-Chloro-1-(3-chlorobenzyl)-1H-benzo[d]imidazol-2-yl)- $\mathrm{N}$-(3,4,5-trimethoxyphenyl)piperidine-1-carboxamide $9\{2,3\}$ 
General Procedure G: 21\{2\} (0.05 g, $0.14 \mathrm{mmol}), 3,4,5$-trimethoxyphenylisocyanate $(0.035 \mathrm{~g}, 0.16 \mathrm{mmol}) .9\{2,3\} 73$ \%; HPLC purity (as area \%) > 92; ${ }^{1} \mathrm{H}$ NMR $\left(400 \mathrm{MHz}, \mathrm{CDCl}_{3}\right) 1.82\left(\mathrm{~m}, \mathrm{br}, 2 \mathrm{H}, \mathrm{CH}_{2} \mathrm{CH}_{2}-\mathrm{NH}\right), 2.10\left(\mathrm{~m}, 2 \mathrm{H}, \mathrm{CH}_{2} \mathrm{CH}_{2}-\mathrm{NH}\right)$, $2.99\left(\mathrm{~m}, 3 \mathrm{H}, \mathrm{CH}_{2}-\mathrm{CH}_{2}-\mathrm{NH}, \mathrm{CH}-\mathrm{CH}_{2}\right), 3.73$ (s, 3H, Ar-OMe), 3.77 (s, 6H, Ar-(OMe) $), 4.12\left(\mathrm{~m}, 2 \mathrm{H}, \mathrm{CH}_{2}-\mathrm{N}\right), 5.27(\mathrm{~s}, 2 \mathrm{H}$, $\left.\mathrm{CH}_{2}-\mathrm{N}\right), 6.31(\mathrm{~s}, 1 \mathrm{H}, \mathrm{N}-\mathrm{H}), 6.59(\mathrm{~s}, 2 \mathrm{H}, \mathrm{Ar}-\mathrm{H}), 6.72(\mathrm{~d}, 1 \mathrm{H}, J$ 7.21, Ar-H), $7.01(\mathrm{~s}, 1 \mathrm{H}, \mathrm{Ar}-\mathrm{H}), 7.13(\mathrm{~m}, 3 \mathrm{H}, \mathrm{Ar}-\mathrm{H}), 7.62(\mathrm{~d}$, $1 \mathrm{H}, 8.33 \mathrm{~Hz}, \mathrm{Ar}-\mathrm{H})$; LC-MS (ESI+) $\mathrm{m} / \mathrm{z} 569[\mathrm{M}+\mathrm{H}]^{+}$;

4-(6-Chloro-1-(3-chlorobenzyl)-1H-benzo[d]imidazole-2-yl)-N-(4-(trifluoromethoxyphenyl)piperidine-1carboxamide $9\{2,4\}$

General Procedure G: $21\{2\}(0.011 \mathrm{~g}, 0.029 \mathrm{mmol})$, 4-trifluoromethoxyphenylisocyanate $(0.004 \mathrm{~g}, 0.032 \mathrm{mmol}) .9\{2,4\}$ 79\%; HPLC purity (as area \%) > 99; ${ }^{1} \mathrm{H}$ NMR (400 MHz ,CDCl $) 1.88\left(\mathrm{~m}, 2 \mathrm{H}, \mathrm{CH}_{2} \mathrm{CH}\right), 2.09\left(\mathrm{~m}, 2 \mathrm{H}, \mathrm{CH}_{2} \mathrm{NCO}\right), 2.99(\mathrm{~m}, 2 \mathrm{H}$, $\left.\mathrm{CH}_{2} \mathrm{NCO}\right), 3.07\left(\mathrm{~m}, 1 \mathrm{H}, \mathrm{CH}_{2} \mathrm{CH}\right), 4.17\left(\mathrm{~m}, 2 \mathrm{H}, \mathrm{CH}_{2} \mathrm{CH}_{2} \mathrm{NCO}\right), 5.31\left(\mathrm{~s}, 2 \mathrm{H}, \mathrm{CH}_{2} \mathrm{Ar}\right), 6.49(\mathrm{~s}, 1 \mathrm{H}, \mathrm{N}-\mathrm{H}), 6.85(\mathrm{~m}, 1 \mathrm{H}, \mathrm{Ar}-\mathrm{H})$, $7.06(\mathrm{~s}, 1 \mathrm{H}, \mathrm{Ar}-\mathrm{H}), 7.14(\mathrm{~d}, 2 \mathrm{H}, J 8.37 \mathrm{~Hz}, \mathrm{Ar}-\mathrm{H}) 7.17(\mathrm{~d}, 1 \mathrm{H}, J 1.8 \mathrm{~Hz}, \mathrm{Ar}-\mathrm{H}), 7.35(\mathrm{~m}, 3 \mathrm{H}, \mathrm{Ar}-\mathrm{H}), 7.38(\mathrm{~d}, 2 \mathrm{H}, \mathrm{J} 8.8 \mathrm{~Hz}, \mathrm{Ar}-$ H), $7.69(\mathrm{~d}, 1 \mathrm{H}, J 8.6 \mathrm{~Hz}, \mathrm{Ar}-\mathrm{H})$; LC-MS (ESI+) $\mathrm{m} / \mathrm{z}=561[\mathrm{M}+\mathrm{H}]^{+}$.

Ethyl 3-(4-(6-chloro-1-(3-chlorobenzyl)-1H-benzo[d]imidazol-2-yl)piperidine-1-carboxamido)benzoate 9\{2,7\}

General Procedure G: 21\{2\} (0.011g, 0.029mmol), ethyl 3-isocyanobenzoate (0.006g, 0.032mmol). 9\{2,7\} 75 \%; HPLC purity (as area \%) > 98; ${ }^{1} \mathrm{H} \mathrm{NMR}\left(400 \mathrm{MHz}, \mathrm{CDCl}_{3}\right) \delta \mathrm{ppm}: 1.40\left(\mathrm{t}, 3 \mathrm{H}, J 7.11 \mathrm{~Hz}, \mathrm{OCH}_{2} \mathrm{CH}_{3}\right), 1.89\left(\mathrm{~m}, 2 \mathrm{H}, \mathrm{CH}_{2} \mathrm{CH}\right), 2.05$ $\left(\mathrm{m}, 2 \mathrm{H}, \mathrm{CH}_{2} \mathrm{NCO}\right), 3.04\left(\mathrm{~m}, 2 \mathrm{H}, \mathrm{CH}_{2} \mathrm{NCO}\right), 3.08\left(\mathrm{~m}, 1 \mathrm{H}, \mathrm{CH}_{2} \mathrm{CH}\right), 4.19\left(\mathrm{~m}, 2 \mathrm{H}, \mathrm{CH}_{2} \mathrm{CH}_{2} \mathrm{NCO}\right), 4.37$ (q, $2 \mathrm{H}, J 7.12 \mathrm{~Hz}$, $\left.\mathrm{OCH}_{2} \mathrm{CH}_{3}\right), 5.35\left(\mathrm{~s}, 2 \mathrm{H}, \mathrm{CH}_{2}\right.$ benzylamine), $6.58(\mathrm{~s}, 1 \mathrm{H}, \mathrm{N}-\mathrm{H}), 6.85(\mathrm{~m}, 1 \mathrm{H}, \mathrm{Ar}-\mathrm{H}), 7.06(\mathrm{~s}, 1 \mathrm{H}, \mathrm{Ar}-\mathrm{H}), 7.21(\mathrm{~d}, 1 \mathrm{H}, \mathrm{J} 1.82$ $\mathrm{Hz}, \mathrm{Ar}-\mathrm{H}), 7.38(\mathrm{~m}, 3 \mathrm{H}, \mathrm{Ar}-\mathrm{H}), 7.71(\mathrm{~m}, 3 \mathrm{H}, \mathrm{Ar}-\mathrm{H}), 7.85(\mathrm{~m}, 1 \mathrm{H}, \mathrm{Ar}-\mathrm{H})$;LC-MS (ESI+) $m / z=549[\mathrm{M}+\mathrm{H}]^{+}$.

Ethyl-4-(4-(6-chloro-1-(3-chlorobenzyl)-1H-benzo[d]imidazole-2-yl)piperidine-1-carboxamido) benzoate $9\{2,8\}$

General Procedure G: $21\{2\}(0.011 \mathrm{~g}, 0.029 \mathrm{mmol})$, ethyl 4-isocyanobenzoate $(0.006 \mathrm{~g}, 0.032 \mathrm{mmol}) .9\{2,8\} 80 \%$; HPLC purity (as area \%) > 98; ${ }^{1} \mathrm{H}$ NMR $\left(400 \mathrm{MHz}, \mathrm{CDCl}_{3}\right) 1.40\left(\mathrm{t}, 3 \mathrm{H}, 7.11 \mathrm{~Hz}, \mathrm{OCH}_{2} \mathrm{CH}_{3}\right), 1.89(\mathrm{~m}, 2 \mathrm{H}, \mathrm{CH} 2 \mathrm{CH}), 2.05(\mathrm{~m}, 2 \mathrm{H}$, $\left.\mathrm{CH}_{2} \mathrm{NCO}\right), 3.04\left(\mathrm{~m}, 2 \mathrm{H}, \mathrm{CH}_{2} \mathrm{NCO}\right), 3.08\left(\mathrm{~m}, 1 \mathrm{H}, \mathrm{CH}_{2} \mathrm{CH}\right), 4.19\left(\mathrm{~m}, 2 \mathrm{H}, \mathrm{CH}_{2} \mathrm{CH}_{2} \mathrm{NCO}\right), 4.37$ (q, 2H, J $\left.7.12 \mathrm{~Hz} \mathrm{OCH}_{2} \mathrm{CH}_{3}\right)$, $5.35\left(\mathrm{~s}, 2 \mathrm{H}, \mathrm{CH}_{2} \mathrm{Ar}\right), 6.58(\mathrm{~s}, 1 \mathrm{H}, \mathrm{N}-\mathrm{H}), 6.85(\mathrm{~m}, 1 \mathrm{H}, \mathrm{Ar}-\mathrm{H}), 7.06(\mathrm{~s}, 1 \mathrm{H}, \mathrm{Ar}-\mathrm{H}), 7.21(\mathrm{~d}, 1 \mathrm{H}, J 1.82 \mathrm{~Hz}, \mathrm{Ar}-\mathrm{H}), 7.38(\mathrm{~m}, 3 \mathrm{H}$, Ar-H), 7.43 (d, 2H, J $8.83 \mathrm{~Hz}, \mathrm{Ar}-\mathrm{H}), 7.69$ (d, 1H, J $8.56 \mathrm{~Hz}, \mathrm{Ar}-\mathrm{H}), 7.97$ (d, 2H, J 8.77 Hz, Ar-H; LC-MS (ESI+) m/z = 549 $[\mathrm{M}+\mathrm{H}]^{+}$.

\section{4-(6-Chloro-1-(3-chlorobenzyl)-1-H-benzo[d]imidazole-2-yl)-N-cyclohexylpiperidine-1-carboxamide 9\{2,10\}}

General Procedure G: 21\{2\} (0.011g, 0.029mmol), cyclohexylisocyanate $(4.13 \mu \mathrm{L}, 0.032 \mathrm{mmol}) .9\{2,10\} 63 \%$; HPC purity (as area \%) > 92; ${ }^{1} \mathrm{H}$ NMR $\left(400 \mathrm{MHz}, \mathrm{CDCl}_{3}\right) 1.12(\mathrm{~m}, 2 \mathrm{H}$, cyclohexyl), $1.15(\mathrm{~m}, 2 \mathrm{H}$, cyclohexyl), $1.39(\mathrm{~m}, 2 \mathrm{H}$, cyclohexyl), $1.82\left(\mathrm{~m}, 2 \mathrm{H}, \mathrm{CH}_{2}-\mathrm{CHN}\right), 1.91\left(\mathrm{~m}, 2 \mathrm{H}, \mathrm{CH}_{2} \mathrm{NCO}\right) 2.11\left(\mathrm{~m}, 2 \mathrm{H}, \mathrm{CH}_{2}-\mathrm{CHN}\right), 2.85\left(\mathrm{~m}, 2 \mathrm{H}, \mathrm{CH}_{2} \mathrm{CH}_{2}\right), 2.93(\mathrm{~m}, 1 \mathrm{H}$, $\left.\mathrm{CHCH}_{2}\right), 3.65\left(\mathrm{~m}, 1 \mathrm{H}, \mathrm{CHCH}_{2}\right), 4.11(\mathrm{~m}, 2 \mathrm{H}), 4.35(\mathrm{~d}, 1 \mathrm{H}, J 7.5 \mathrm{~Hz}, \mathrm{~N}-\mathrm{H}), 5.35\left(\mathrm{~s}, 2 \mathrm{H}, \mathrm{CH}_{2} \mathrm{~N}\right), 6.90(\mathrm{br} \mathrm{d}, 1 \mathrm{H}, J 6.90 \mathrm{~Hz}, \mathrm{Ar}-$ $\mathrm{H}$ benzimidazole), 7.12 (br s, 1H, Ar-H benzylamine), 7.21 (d, 1H, J $1.7 \mathrm{~Hz}, \mathrm{Ar}-\mathrm{H}), 7.31$ (m, 3H, Ar-H benzylamine), 7.8 (d, $1 \mathrm{H}, \mathrm{J} 9.0 \mathrm{~Hz}, \mathrm{Ar}-\mathrm{H})$; LC-MS (ESI+) $\mathrm{m} / \mathrm{z}=483[\mathrm{M}+\mathrm{H}]^{+}$.

Ethyl 4-(4-(6-chloro-1-(3-methoxybenzyl)-1H-benzo[d]imidazol-2-yl)piperidine-1-carboxamido)benzoate 9\{3,8\}

General Procedure G: 21\{3\} (0.01 g, 0.028 mmol), ethyl 4-isocyanobenzoate (0.006 g, $0.031 \mathrm{mmol}) .9\{3,8\}$ 68\%; HPLC purity (as area \%) > 99; ${ }^{1} \mathrm{H}$ NMR $\left(400 \mathrm{MHz}, \mathrm{CDCl}_{3}\right) 1.40\left(\mathrm{t}, 3 \mathrm{H}, \mathrm{J} 7.11 \mathrm{~Hz}, \mathrm{OCH}_{2} \mathrm{CH}_{3}\right), 1.96(\mathrm{~m}, 2 \mathrm{H}, \mathrm{CH} 2 \mathrm{CH}), 2.08(\mathrm{~m}, 2 \mathrm{H}$, $\mathrm{CH}_{2} \mathrm{CH}$ ), $3.04\left(\mathrm{~m}, 3 \mathrm{H}, \mathrm{CH}_{2} \mathrm{CH}\right.$ ), 3.79 (s, 3H, OMe), 4.21 (m, 2H, $\left.\mathrm{CH}_{2} \mathrm{CH}_{2} \mathrm{NCO}\right), 4.35$ (q, 2H, J $\left.7.1 \mathrm{~Hz} \mathrm{CH}_{2} \mathrm{CH}_{3}\right), 5.34$ (s, 2H, $\left.\mathrm{CH}_{2} \mathrm{Ar}\right), 6.61(\mathrm{~m}, 3 \mathrm{H}, \mathrm{NHCO}, \mathrm{Ar}-\mathrm{H}), 6.85(\mathrm{~m}, 1 \mathrm{H}, \mathrm{Ar}-\mathrm{H}), 7.21(\mathrm{~m}, 3 \mathrm{H}, \mathrm{ArH}), 7.48(\mathrm{~d}, 2 \mathrm{H}, \mathrm{Ar}-\mathrm{H}), 7.68(\mathrm{~d}, 1 \mathrm{H}, J 7.8 \mathrm{~Hz}, \mathrm{Ar}-$ H), 7.91 (d, 2H, J $8.30 \mathrm{~Hz}$, Ar-H); LC-MS (ES+) $m / z=547[\mathrm{M}+\mathrm{H}]^{+}$. 
Ethyl 3-(4-(6-chloro-1-(3-methoxybenzyl)-1H-benzo[d]imidazol-2-yl)piperidine-1-carboxamido) benzoate $9\{3,7\}$

General Procedure G: 21\{3\} (0.01 g, 0.028 mmol), ethyl 4-isocyanobenzoate (0.006 g, $0.031 \mathrm{mmol})$. 9\{3,7\} 72\%; HPLC purity (as area \%) > 99; ${ }^{1} \mathrm{H} N M R\left(400 \mathrm{MHz}, \mathrm{CDCl}_{3}\right) 1.39\left(\mathrm{t}, 3 \mathrm{H}, \mathrm{J} 7.11 \mathrm{~Hz}, \mathrm{OCH}_{2} \mathrm{CH}_{3}\right), 1.91(\mathrm{~m}, 2 \mathrm{H}, \mathrm{CH} 2 \mathrm{CH}), 2.11(\mathrm{~m}, 2 \mathrm{H}$, $\mathrm{CH}_{2} \mathrm{CH}$ ), $3.04\left(\mathrm{~m}, 3 \mathrm{H}, \mathrm{CH}_{2} \mathrm{NCO}\right), 3.78(\mathrm{~s}, 3 \mathrm{H}, \mathrm{OMe}), 4.22 \mathrm{~Hz}\left(\mathrm{~m}, 2 \mathrm{H}, \mathrm{CH}_{2} \mathrm{CH}_{2} \mathrm{NCO}\right), 4.39$ (q, 2H, J 7.13 Hz, $\left.\mathrm{CH}_{2} \mathrm{CH}_{3}\right), 5.34$ $\left(\mathrm{s}, 2 \mathrm{H}, \mathrm{CH}_{2} \mathrm{Ar}\right), 6.57(\mathrm{~m}, 3 \mathrm{H}, \mathrm{NHCO}, \mathrm{ArH}), 6.87(\mathrm{~m}, 1 \mathrm{H}, \mathrm{ArH}), 7.20(\mathrm{~m}, 2 \mathrm{H}, \mathrm{ArH}), 7.45(\mathrm{~m}, 2 \mathrm{H}, \mathrm{ArH}) 7.70(\mathrm{~m}, 3 \mathrm{H}, \mathrm{ArH})$, $7.81(\mathrm{~s}, 1 \mathrm{H}, \mathrm{ArH}) ; \mathrm{LC}-\mathrm{MS}(\mathrm{ESI}+) \mathrm{m} / z 547[\mathrm{M}+\mathrm{H}]^{+}$.

4-(6-Chloro-1-(3-methoxybenzyl)-1H-benzo[d]imidazole-2yl)-N-(4-(trifluoromethoxy)phenyl)piperidine-1carboxamide $9\{3,4\}$

General Procedure G: 21\{3\} (0.01 g, $0.028 \mathrm{mmol})$, 4-trifluoromethoxyphenylisocyanate $(0.005 \mathrm{~g}, 0.031 \mathrm{mmol}) .9\{3,4\}$, 41\%; HPLC purity (as area \%) > 96; ${ }^{1} \mathrm{H}$ NMR $\left(400 \mathrm{MHz}, \mathrm{CDCl}_{3}\right) 1.99\left(\mathrm{~m}, 2 \mathrm{H}, \mathrm{CH}_{2} \mathrm{CH}\right), 2.08(\mathrm{~m}, 2 \mathrm{H}, \mathrm{CH} 2 \mathrm{CH}), 3.02(\mathrm{~m}, 3 \mathrm{H}$, $\left.\mathrm{CH}_{2} \mathrm{NCO}\right), 3.77(\mathrm{~s}, 3 \mathrm{H}, \mathrm{OMe}), 4.19 \mathrm{~Hz}\left(\mathrm{~m}, 2 \mathrm{H}, \mathrm{CH}_{2} \mathrm{CH}_{2} \mathrm{NCO}\right), 5.34\left(\mathrm{~s}, 2 \mathrm{H}, \mathrm{CH}_{2} \mathrm{Ar}\right), 6.57(\mathrm{~m}, 3 \mathrm{H}, \mathrm{NHCO}, \mathrm{ArH}), 6.86(\mathrm{~m}, 1 \mathrm{H}$, $\operatorname{ArH}), 7.14(\mathrm{~d}, 2 \mathrm{H}, J 8.4 \mathrm{~Hz}, \mathrm{ArH}), 7.21(\mathrm{~m}, 3 \mathrm{H}, \mathrm{ArH}), 7.35(\mathrm{~d}, 2 \mathrm{H}, J 8.5 \mathrm{~Hz}, \operatorname{ArH}), 7.66(\mathrm{~m}, 2 \mathrm{H}, \mathrm{Ar}-H)$; LC-MS (ESI+) $\mathrm{m} / \mathrm{z}=$ $559[\mathrm{M}+\mathrm{H}]^{+}$.

$N$-(4-Acetylphenyl)-4-(6-chloro-1-(3-methoxybenzyl)-1H-benzo[d]imidazole-2-yl)piperidine-1-carboxamide 9\{3,2\}

General Procedure G: 21\{3\} (0.01 g, 0.028 mmol), 4-acetylphenylisocyanate (0.004 g, 0.024 mmol). 9\{3,2\} 67\%; HPLC purity (as area \%) > 99; ${ }^{1} \mathrm{H} N M R\left(400 \mathrm{MHz}, \mathrm{CDCl}_{3}\right) 1.97\left(\mathrm{~m}, 2 \mathrm{H}, \mathrm{CH}_{2} \mathrm{CH}\right), 2.11\left(\mathrm{~m}, 2 \mathrm{H}, \mathrm{CH}_{2} \mathrm{CH}\right), 2.48\left(\mathrm{~s}, 3 \mathrm{H}, \mathrm{CH}_{3}\right), 3.02$ (m, 3H, CH $\mathrm{CHCO}_{2}, 3.72(\mathrm{~s}, 3 \mathrm{H}, \mathrm{OMe}), 4.19 \mathrm{~Hz}\left(\mathrm{~m}, 2 \mathrm{H}, \mathrm{CH}_{2} \mathrm{CH}_{2} \mathrm{NCO}\right), 5.35$ (s, 2H, $\mathrm{CH}_{2}$ benzylamine), $6.57(\mathrm{~m}, 3 \mathrm{H}, 13 \mathrm{~Hz}$, $\mathrm{N}$-H-urea, Ar- H-OMe, Ar- H-OMe), $6.86(\mathrm{~m}, 1 \mathrm{H}, 7.13 \mathrm{~Hz}, \mathrm{Ar}-\mathrm{H}-\mathrm{Cl}), 7.22(\mathrm{~m}, 3 \mathrm{H}, 3-8 \mathrm{~Hz} \mathrm{Ar}-\mathrm{H}), 7.49(\mathrm{~d}, 2 \mathrm{H}, 8.7 \mathrm{~Hz}, \mathrm{Ar}-\mathrm{H}-$ $\left.\mathrm{COCH}_{3}\right), 7.71$ (d, $\left.1 \mathrm{H}, 9.2 \mathrm{~Hz} \mathrm{Ar-H}-\mathrm{Cl}\right), 7.99$ (d, 2H, $8.68 \mathrm{~Hz} \mathrm{Ar-H-COCH}$ ). LC-MS (ES+) m/z 517 [M+H] .

4-(6-Chloro-1-(4-methoxybenzyl)-1H-benzo[d]imidazol-2-yl)- $\mathbf{N}$-(4-nitrophenyl)piperidine-1-carboxamide $\mathbf{9}\{4,1\}$ General Procedure G: $21\{4\}$ (0.05g, 0.14mmol), paranitrophenylisocyanate (0.025g, 0.16mmol.). 9\{4,1\} 63\%; HPLC purity (as area \%) > 89; ${ }^{1} \mathrm{H} \mathrm{NMR}\left(400 \mathrm{MHz}, \mathrm{CDCl}_{3}\right) 1.93$ (d, br, 2H, J 14.79, $\left.\mathrm{CH}_{2}-\mathrm{NC}(\mathrm{O})\right), 2.13\left(\mathrm{~m}, \mathrm{br}, 2 \mathrm{H}, \mathrm{CH}_{2} \mathrm{CH}_{2}-\mathrm{N}\right.$ ), $3.12\left(\mathrm{~m}, \mathrm{br}, 2 \mathrm{H}, \mathrm{CH}_{2}-\mathrm{CH}_{2}-\mathrm{N}\right.$ ), 3.73 (s, 3H, Ar-OMe), 3.77 (s, 3H, Ar-OMe), 4.11 (br d, 2H, J 13.34, $\mathrm{CH}_{2}-\mathrm{CH}_{2}-\mathrm{N}$ ), 5.28 (s, $\left.2 \mathrm{H}, \mathrm{N}-\mathrm{CH}_{2}(\mathrm{Ar})\right), 6.66(\mathrm{~s}, 1 \mathrm{H}, \mathrm{N}-\mathrm{H}-\mathrm{Ar}), 6.89$ (d, 2H, J 8.61, 2H, Ar-H), 6.99 (d, 2H, J 8.73, Ar-H), 7.24 (d, 1H, J 2.5, Ar-H), 7.30 (s, 1H, Ar-H), 7.50 (d, 2H, J 8.19, Ar-H), 7.69 (d, 1H, Ar-H), 8.22 (d, 2H, J 8.19, Ar-H); LC-MS (ESI+) m/z 520 $[\mathrm{M}+\mathrm{H}]^{+}$.

N-(4-Acetylphenyl)-4-(6-chloro-1-(4-methoxybenzyl)-1H-benzo[d]imidazol-2-yl)piperidine-1-carboxamide $9\{4,2\}$ General Procedure G: $21\{4\}(0.097 \mathrm{~g}, 0.27 \mathrm{mmol})$, 4-acetylphenylisocyanate $(0.074 \mathrm{~g}, 0.46 \mathrm{mmol})$. Chromatography (50\% EtOAc, petrol), 9\{4,2\} $55 \%$; HPLC purity (as area \%) > 96; ${ }^{1} \mathrm{H} \mathrm{NMR}\left(400 \mathrm{MHz}, \mathrm{CDCl}_{3}\right) 1.82\left(\mathrm{~m}, 2 \mathrm{H}, \mathrm{CH}_{2} \mathrm{CH}_{2}-\mathrm{NH}\right)$, $2.05\left(\mathrm{~m}, 2 \mathrm{H}, \mathrm{CH}_{2} \mathrm{CH}_{2}-\mathrm{NH}\right), 2.48\left(\mathrm{~s}, 3 \mathrm{H}, \mathrm{Ar}-\mathrm{COCH}_{3}\right) 2.94\left(\mathrm{~m}, 3 \mathrm{H}, \mathrm{CH}_{2}-\mathrm{CH}_{2}-\mathrm{NH}\right.$ and $\left.\mathrm{CH}_{-} \mathrm{CH}_{2}\right), 3.71$ (s, 3H, Ar-OMe), $4.12(\mathrm{~m}$, br $\left.2 \mathrm{H}, \mathrm{CH}_{2}-\mathrm{N}\right), 5.22\left(\mathrm{~s}, 2 \mathrm{H}, \mathrm{CH}_{2}-\mathrm{N}\right), 6.62(\mathrm{~s}, 1 \mathrm{H}, \mathrm{N}-\mathrm{H}), 6.79(\mathrm{~d}, 2 \mathrm{H}, J$ 8.7, Ar-H), $6.84(\mathrm{~d}, 2 \mathrm{H}, J$ 8.7, Ar-H), $7.18(\mathrm{~m}, 2 \mathrm{H}, \mathrm{Ar}-$ H), 7.39 (d, 2H, J 8.8, Ar-H), 7.6 (dd, 1H, J 8.1, 1.04, Ar-H), 7.89 (d, 2H, J 8.8, Ar-H).; LC-MS (ESI+) m/z 517 [M+H].

4-(6-Chloro-1-(4-methoxybenzyl)-1H-benzo[d]imidazol-2-yl)- $\mathrm{N}$-(3,4,5-trimethoxyphenyl)piperidine-1-carboxamide $9\{4,3\}$

General Procedure G: $21\{4\}(0.05 \mathrm{~g}, 0.14 \mathrm{mmol}), 3,4,5$-trimethoxyphenylisocyanate $(0.033 \mathrm{~g}, 0.16 \mathrm{mmol}) .9\{4,3\}$ 61\%; HPLC purity (as area \%) > 94; ${ }^{1} \mathrm{H}$ NMR (400 MHz, CDCl $) 1.91$ (d, br, $\left.2 \mathrm{H}, J 14.75, \mathrm{CH}_{2}-\mathrm{NC}(\mathrm{O})\right), 2.11\left(\mathrm{~m}, \mathrm{br}, 2 \mathrm{H}, \mathrm{CH}_{2} \mathrm{CH}_{2}-\mathrm{N}\right.$ ), $3.02\left(\mathrm{~m}, \mathrm{br}, 2 \mathrm{H}, \mathrm{CH}_{2}-\mathrm{CH}_{2}-\mathrm{N}\right.$ ), 3.82 (s, 3H, Ar-OMe), 3.84 (s, 3H, Ar-OMe), 3.88 (s, 6H, Ar-OMe), 4.11 (d, br, 2H, J 
13.34, $\mathrm{CH}_{2}-\mathrm{CH}_{2}-\mathrm{N}$ ), 5.32 (s, 2H, N-CH$(\mathrm{Ar})$ ), 6.29 (s, 1H, N-H-Ar), 6.88 (d, J 8.61, 2H, Ar-H), 6.98 (d, 2H, J 8.73, Ar-H), 7.24 (d, 1H, J 2.5, Ar-H), 7.26 (s, 1H, Ar-H), 7.69 (d, 1H, Ar-H); LC-MS (ESI+) m/z $565[\mathrm{M}+\mathrm{H}]^{+}$. 